\title{
SIC 2,1982
}

University of Linköping Studies in Communication

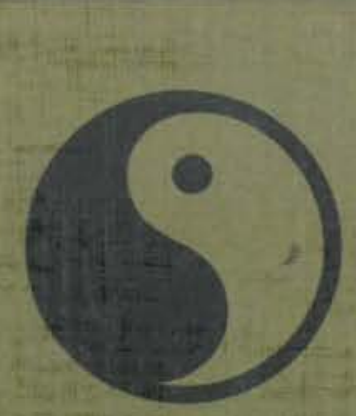

The Written Language Bias in Linguistics

Per Linell 
THE WRITTEN LANGUAGE BIAS IN LINGUISTICS

Per Linell

University of Linköping

Dept. of Communication Studies

SIC 2, 1982

LiU-Tema K-RB-82-2

ISSN $0280-5634$

ISBN $91-7372-587-0$

Address: Department of Communication Studies University of Linköping S-581 83 LINKOPING

Sweden 

SIC - Studies in Communication - is a series of reports and working papers produced by the Department of Communication Studies, University of Linköping, Sweden. SIC will appear irregularly. Most reports will be written in English, although some may appear in Swedish or other Scandinavian languages.

SIC will publish articles, reviews and monographs which are the outcome of research carried out in our department. Some issues will contain papers that have been presented at conferences arranged by the department. Many of the reports published in SIC will appear in final versions elsewhere. We hope that our reports will elicit discussion and comment from colleagues in the scientific fields which are relevant for our interdisciplinary program, the study of human communication. Our SIC reports are distributed on an exchange basis to other institutions and scholars. Copies may also be purchased from our department.

Linköping, 1982

Copyright Department of Communication Studies 


\section{PREFACE}

For some time I have been thinking of the predicament of present-day linguistics in terms of what I venture to call "the written language bias". It seems to me that a great number of our explicit or implicit theories, our methods and preferences are heavily influenced by the very long traditions of analyzing mainly, or only, certain kinds of written language. Even when we are in fact focussing on spoken language, we seem to approach it with a theoretical apparatus which is more apt for the analysis of written language. This essay represents a first attempt on my part to systematize some thoughts about this. I hope that it will stimulate discussion and lead to constructive criticism.

This book was largely written in the academic year $1980 / 81$ when I was employed by the Swedish Research Council for the Humanities and Social Sciences. When I worked at it, I profited from many ideas and suggestions of other people. Among these scholars I want to single out two, Jens Allwood and Ragnar Rommetveit, with whom I had many inspiring discussions.

I would also like to express my gratitude to Marianne Axelson, Solweig Bladh and Britt-Marie Genet who typed my manuscripts, and to Brian Beattie who checked and corrected my English.

Linköping, September 1982

Per Linell 
TABLE OF CONTENTS

I. INTRODUCTION

II. SPEECH AND WRITING 5

1. Spoken language and speech communication 5

2. Communication by written texts 7

III. THE SOURCES OF THE WRITTEN LANGUAGE BIAS IN LINGUISTICS

1. Technology 14

2. The traditional tasks of linguistics 21

3. The status of written language 25

4. Written language as metalanguage 29

IV. THE WRITTEN LANGUAGE BIAS IN LINGUISTICS: ON THE SCOPE OF OUR CLAIMS

v. LINGUISTICS AND THE OVERALL THEORY OF LANGUAGE 35

1. Linguistics and other language sciences $35^{\text {th }}$

2. Structure and use: Language vs parole,
competence vs performance

3. Language as a set of products 45

$\begin{array}{ll}\text { 4. Inguistic structures as closed hierarchical } & \\ \text { systems of things } & 47\end{array}$

5. The invariance of linguistic structure 52 .

6. The atemporality of linguistic structure 55

7. The autonomy of language and linguistic
structure

8. Norms and normativity in linguistics 59

VI. GRAMMAR 63

1. The concept of sentence 63

2. The neglect of prosody 72

3. The concept of grammaticality 75

4. Words 83

5. The structure of texts and discourse 87 
VII. SEMANTICS

1. Introduction: The autonomy of Iinguistic meaning

2. Word meanings

3. The boundary between semantics and pragmatics

4. Semantics and truth

5. Speech acts, sentence meaning and utterance meaning

6. The interpretation of utterances: The roles of the speaker, the listener, and the outside observer

7. The interpretation of written texts

8. Logic and formal languages

9. Semantic representations

10. Knowledge structures

11. Semantic representations and thought processes

VIII. PHONOLOGY

1. Phonological structure

2. L'image acoustique

3. Phonological rules

4. The relation between phonology and phonetics

1. Communication as transportation of messages

2 . The functions of language

3. Thought and expression: Content and form

4. Social and individual aspects of language

5. Semiotics

$x$. LANGUAGE ACQUISITION

1. Phonological development

2 . The innate faculty of language 
XI. IINGUISTIC VARIATION

1. The neglect of linguistic variation

2. The depreciation of spoken vernaculars

3 . The concept of an ideal language

XII. EPILOGUE 

I. INTRODUCTION

"To exist is to be a thing or an object" (Stenlund 1980:86, my translation)

In this book the modern linguist's view on language is discussed from a rather unusual point of view. It is argued that our conception of language is deeply influenced by a long tradition of analyzing only written language, and that modern linguistic theory, including psycholinguistics and sociolinguistics, approaches the structures and mechanisms of spoken language with a conceptual apparatus, which - upon closer scrutiny - turns out to be more apt for written language in surprisingly many and fundamental aspects. I will refer to this situation as the written language bias in linguistics.

My focus is on the predicaments of present-day linguistics. Since my background is that of an ordinary linguist rather than that of a historian of linguistic science, I cannot undertake the enormous task of tracing the ideological and social history behind current linguistic theory. However, I will indicate the extent to which linguistics, like most other sciences, is still dependent on important events of the past, both technological inventions - in our case the development of alphabetical writing, book printing and today's computers - and practical political goals and social concerns that have motivated the practice of linguistic science since antiquity.

Our conception of linguistic behavior is biased by a tendency to treat processes, activities, and conditions on them in terms of object-like, static, autonomous and permanent structures, i.e., as if they shared such properties with written characters, words, 
texts, pictures and images. Though my discussion will be confined to linguistics, I am well aware that the same type of bias can be found in many other sciences. Thus, the history of psychology illustrates very well the very strong tendency to reify mental, or mentally governed, processes, actions and behaviours; compare the common analyses in terms of images (sensory images, memory images), mental representations (e.g. of knowledge), engrams and various other kinds of structures. Cognitive psychology has exploited a great many metaphors depicting memories as objects stored in a mind space, and the process of retrieval has been conceived as a search for these objects (Roedinger 1980). In general, most of Western philosophy and science has been stuck with the metaphysical assumption that the world is made up of "things" or "objects". Thinking in terms of things le.g. Sten1und $1980: 86,98-9,121,148$ ) has a very long tradition, which only recently and very reluctantly has been seriously questioned in natural science. Interestingly enough, one may venture to propose that this whole philosophy may be related to the structure of (certain) natural languages, 1.e. the fact that everything that we talk about (topics, "logical subjects") tends to be designated by nouns and, therefore, assumes the character of "primary substances" (in Aristotle's sense),

Pictures and other static models ("object models") have deeply influenced thinking in many sciences. In the words of Walter Ong:

"Milič Capek's book The Philosophical Impact of Contemporary Physics (1961) calls for physicists to supplement their view of the world as basically a "picture", which is certainly not all it is, and to avail themselves of auditory phenomena, with their strikingly dynamic character, as models of physical phenomena, so as to open the way out of certain dead-ends in present physical sciences."

(Ong 1974:169)

We shall not probe the question of how true Ong's picture (sic!) of the natural sciences is. As I have already stated, this book will deal with people's thinking about language. If we focus 
on linguistics and philusophy we will see that in these the object metaphor is very pervasive. It is applied at all levels of linguistic structure ${ }^{l i}$, e.g.:

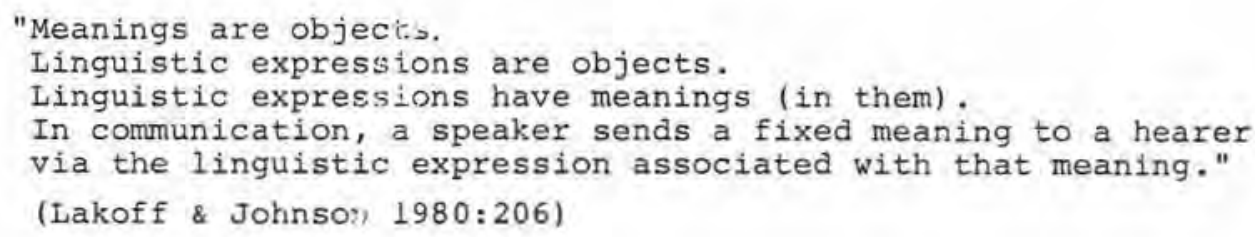

Metaphorical concept: are a special type of concepts; they are understood in teras of other concepts, as if the things to be understood were of he same nature as the things they are compared to (i.e. metapho cally related to). But our thinking is easily caught by the henitual use of such metaphors - Lakoff \& Johnson (1980) argue thy: a very large part of our language use and our thinking is bas vally metaphorical - and we may soon begin to think that meanings (etc) are indeed (abstract) objects, and that they cannot be explicated in other terms. (This will be discussed at some length below, especially in sections VII.1-2, VII.9-10.) The main point of this book, however, is that our most common ways of thinking about language in general, our most cherished metaphors, are, if not wholly derived from, at least heavily influenced by our time-honored traditions of dealing with language mostly, and often exclusively, in its written forms.

1) Note the metaphorical content of "structure", "level", and many other concepts commonly used in linguistics. 


\section{SPEECH AND WRITING}

\section{II,1 Spoken language and speech communication}

In a normal speech communication situation, a speaker tries to exert an influence on a listener (or a group of listeners) by making him (or them) perceive, understand, feel or do something particular. The speaker guides the listener into doing this by exposing a linguistically structured speech behaviour, which operates together with non-verbal signals, various kinds of background knowledge that the speaker and the listener have, the listener's responses and other characteristics of the physical and social context in which the communicative activities are embedded. The variouo behavioral and information-processing operations involved in both the production and comprehension of speech are transient events which, in addition, partially overlap and occur at very high rates. There is often a frequent exchange of turns (i.e. speaking vs listening turns) between the communicating parties. All in all, this brings about a very intricate and rapidy evolving social interaction between the parties.

We can briefly state some of the most important features of speech communication in the following points:

1. Speech is a dynamic, ephemeral behavior distributed in time; it proceeds continuously and its inherent dynamics, the changes at various levels, must be subject to on-line monitoring and analysis by both communicating parties; as one goes on, one can no longer observe that which was produced earlier. The products of the speaker's activities (behavioral movements and sound waves) fade rapidly over a period of time, and the same applies to the listener's activities. (I disregard hare the fact that some types of "products" remain in short-term memory for certain limited periods of time.) This naturally leads to focusing on the dynamic behavior as such rather than on some persistent products (such as those in writing). 
2. Speech behavior has many features of continuous movements (rather than a chain of successive states).

3. The whole interaction between speaker and listener is dependent on the situation (context) in many extremely important ways.

First of all, the speaker's speech behavior is continuously accompanied and supplemented (occasionally contradicted) by various non-verbal signals, which means that the verbal message as such is often much less explicit than in writing; referents may be pointed to, interpretations may be made more precise and complex through gestures, facial expressions, tones of voice etc.

After all, the use of an utterance in a normal situation involving face-to-face interaction is not an isolated speech act; it is part of a comprehensive communicative act which comprises the use of both verbal means (speech) and nonverbal means (gesticulation etc). The message is conveyed, or shown, in several ways simultaneously, and the role played by spoken language cannot be properly understood without taking into consideration the whole communicative act.

Secondly, both speaker and listener are normally physically present at the same place, and they normally have a considerable amount of background knowledge about each other, the things talked about etc. Parts of this knowledge may be shared by both interlocutors.

Thirdly, the listener responds all the time (verbally and, perhaps most importantly, by non-verbal means), and this feedback continuously influences the speaker's behavior. The speaker must produce his utterances quickly and readily, and the listener must respond just as rapidly, under the pressure of the emotive and social atmosphere of the face-to-face interaction. 
In short, these various feature imply that dialogues, which are the typical application of speech, must be regarded as a complex social interplay betwe $n$ agents.

4. Communication through speech ; a resource available for all normally equipped human being: across different social groups and cultures. It is acquired under rather different conditions than writing. Its ontogenesis is part of the normal individual's primary socialization, which starts and largely develops in early childhood as an jntegrated element of habitual activities in everyday culture. ro a large extent it then remains a feature of the private sphere of people's lives. Knowledge of one's spoken language is an inalienable element of one's knowledge of everyday culture (cf. Berger \& Luckmann 1967).

\section{II,2 Communication by written texts}

Unlike speech, written texts are typically not perceived and interpreted at the same times and places as they are produced. The analysis of written language - both by linguists and normal users (readers) - necessarily focusses on the products of the writer's activities, i.e. on the written texts, whereas the production process itself is non-accessible and unimportant for the normal reader. However, while the processes involved in the production of written texts are usually not directly communicatively significant, the fact that the products persist over time makes various types of intermediary communicative acts available. The written texts can be used in different ways, reemployed, duplicated, distributed to particular persons or groups in new situations, and these activities can be regarded as proper communicative acts in their own right (or as parts of such acts). Note, however, that these acts are normally instigated and performed by other people than the writer (the original sender) himself. While a speaker may exert a considerable social-psychological pressure on the listener and may direct the latter's thoughts and feelings through his own verbal (and non-verbal) signals, a writer has not at all the same immediate power over 
the reader(s). If we proceed further in comparing communication by written texts to communication in spoken discourse, we will also note the following characteristics (cf II.1).

1. A written text and its components parts (letters, words, sentences, paragraphs etc) have the character of objects; they are persistent and static (atemporal) (spatially but not temporally organized). Considerable sections may be scanned (almost) simultaneously or at least repetitively (in principle as many times as required). (I disregard here the fact that the activities involved in reading are also dynamic and distributed in time, something which must have consequences for the resulting comprehension.) Rapid, urgent responses are usually not necessary.

2. The written text is made up of discrete symbols, i.e. letters (at least in print) and (graphic) words, and these are organized in certain regular spatial patterns (according to syntactic rules as well as various conventions of punctuation and paragraph division). (These symbols are the approximate counterparts of only some of the structural (i.e. segmentalphonological, grammatical, lexicall features of spoken language; the prosodic features and the non-verbal signals of the communicative acts in speech situations have almost no correspondence in writing).

3. Unlike spoken utterances, a written text lacks an immediate context. Though it is true that a reader must, in order to properly understand a written text "place it in a wider context" (using various kinds of background knowledge, e.g. knowledge about the topics of the text, assumptions regarding the writer's intentions), a written text is - as a rule and in comparison with spoken utterances - relatively explicit (the absence of an immediate context must be compensated for, i.e., referents must be more fully described, arguments must be represented more extensively) and relatively autonomous or context-free (the text stands on its own feet to a much 
greater extent than spoken utterances in a dialogue, for which the sender's and the receiver's behavior, expectations, intentions etc are normally immediately relevant for the interpretation). In principle a written text can be decoded at any place, and the decoding can often be performed by a great number of different people.

Furthermore, the medium of writing is adapted for a monologic function. Normally, the sender, the writing individual, works alone, and the same applies to the receiver.

4. The acquisition of the ability to read and write is quite different from learning to speak and understand speech, Normally, a considerable amount of explicit instruction is needed, and the more skilled and erudite writers have usually gone through many years of rather intense training. Thus, the acquisition of written language belongs to the so-called secondary socialization, in which school and other cultural institutions play a very important instrumental part. Schooling and education are unevenly distributed in most (all?) societies. Thus, while spoken language is largely every man's property, written language is the belonging of only rather few people. This circumstance forms the basis of the function of written language in social stratification (III.3). Written language is mainly used in the non-private life sphere, and, again unlike spoken language, it is not integrated with everyday knowledge and culture but is associated mostly with various kinds of abstract knowledge separate from the world of direct experience.

When writing is taught, a number of more or less explicit norms or rules are referred to, and these norms will therefore be partly conscious to the language users. This in turn is related to still other importantproperties of written language:

a) written language is more constrained by rules and conventions than spoken language, especially as regards its form. 
b) in general, there is less variation (i.e., less dialectal and idiolectal variation) and more invariance in written language, except perhaps in advanced literacy uses, especialIy poetry.

c) the conditions under which written language is generally taught have promoted the quite common belief that (some variants of) written language represent(s) the "grammatical", correct language, whereas many variants of spoken language are incorrect, defective, incoherent, ugly and/or rude.

It must be admitted, of course, that the differences between spoken and written language are not always and everywhere very clear-cut. There are spoken genres, in which language is used very much as in certain written styles, and, conversely, writing can sometimes be deliberately used for mirroring certain speech styles. Moreover, historically, there must have existed transitory forms; how else could we explain the invention and development of written languages in cultures that were originally entirely oral in nature?

More specifically, if we consider the fourth point of above, there are of course variations in normativity and ritualization in spoken language too. In particular, there are often certain bound forms of speech, which are more conventionally constrained in form and content than normal spoken discourse. Such varieties are often used for the recital of orally downtraded myths, laws, proverbs, epic poems etc, and they seem to occur also in cultures which totally lack writing (e.g., certain Polynesian cultures). On the other hand, these varieties are among those which are liable to be written down at an early stage in those cultures where writing systems are indeed developed.

Thus, we can say that certain features which we ascribe to written language have their natural counterparts in certain spoken genres. But I would still maintain that writing as such hashad a profound influence on our thinking, since it always 
transforms the structure of language and gives prominence to certain features. This then creates a special type of background for the development of linguistic theory; a theory of written language cannot, and should not, be entirely identical with a corresponding theory of spoken language ${ }^{2)}$.

2) I abstain here from discussing the conditions under which communication by means of spoken language takes place when modern technical equipment (e.g. recording on sound and/or video tapes) and mass media (e.g. radio and television) are involved. obviously, some of the features that I listed earlier as typical of speech communication are no longer present then. Furthermore, a fair amount of the speech broadcast through the media (and in other non-private situations) is heavily dependent on written texts; often, people simply read their typescripts aloud. 

III. THE SOURCES OF THE WRITTEN LANGUAGE BIAS IN LINGUISTICS

\begin{abstract}
"European linguistic thought formed and matured over concern with the cadavers of written languages; almost all its basic categories, its basic approaches and techniques were worked out in the process of reviving these cadavars."
\end{abstract}

(Voloక̌inov 1973:71)

Traditionally linguists have been occupied with written language to a much greater extent than with spoken language. Even today, when much lip service is being paid to the need to study spoken language, we approach language with a conceptual apparatus which shows numerous signs of being derived from this tradition. This I will demonstrate later on (sections V-XI). At the outset, it would not be out of place, however, to ask the question how and why linguistics became biased towards written language in the first place. I am of course far from being able to account for the entire history here, but I will nevertheless suggest four factors which it would seem necessary to consider in this context.

The four points, which, by the way, are not mutually independent, are these:

a) the technological evolution of the medium of written language as such, which has determined important aspects of our conception of what language in general is or should be,

b) the motives and goals behind the activities of linguists in the past. These goals are no longer so dominant today, but the traditional directions that linguistics took as a consequence of them still characterize our theories and practice.

c) the high status of written language in almost all societies and therefore also among linguists belonging to those societies,

d) the role of written language as the metalanguage of linguistic description. Thus, written language was not only the subject of scientific analysis, it was also (and still is) the medium in which the products of the analysis, our various theories and metalinguistic statements, were stated. 
The first two points merely follow the general tendencies of sciences to adjust to technological and practical-political needs and possibilities. The latter points, (c-d) are more specific to linguistics, although they too have repercussions on all scientific and other activities which are dependent in some way or another on the existence of writing.

\section{III.1 Technology}

It is a commonplace that technology always tends to have an impact on what kinds of theories scientists develop and what kinds of goals and problems they set up in their enterprises. Technology leads to new cultural advances. No wonder then that technological devices and their preconditions and inherent possibilities are regarded as particularly important and interesting by society in general, and hence also by scientists. Perhaps the gradual evolution of writing represents the most important technological advance of all. It makes civilization and more advanced culture possible, and therefore it is in fact a precondition for all other technological advances so far made. One is nevertheless justified in saying that contemporary linguists do not in general have any deeper appreciation of the influence that the medium of writing has had on their own science. Yet, what has happened is that, for the reasons I have just outlined, linguists have been occupied with explicating certain properties and inherent possibilities of written language, and these properties have then very often rather naively - been assumed to be characteristic of language in general.

There are at least three important stages in the development of writing:a) the first picture-based writing systems, b) the syllabaries and alphabetic writing systems which were based on the phonetic form of the corresponding spoken words and utterances, and c) printing, which made large-scale distribution of written works possible. As a fourth milestone of similar importance one may consider today's rapid development of computers. Basically what these perform are very sophisticated manipulations of symbols which are or can be written (written out). 
Olson (1977) has described the development of writing techniques as a gradual transition from the context-boundness of spoken language to successively higher levels of autonomy of written messages. An oral message is bound to the time and place where it is uttered. The outer form, the speech behavior, is a very ephemeral phenomenon, and the semantic interpretation is crucially dependent on various factors in the communication situation, factors which may be considered extrinsic to language as such. Thus, oral language is far from being autonomous (II.1).

When man began to write down symbols of various types, this meant that the products of the sender's activities were made permanent, and this, 01son argues, is the first step towards an autonomy of the text.

However, as long as the written symbols were basically pictures of some kind, there were heavy restrictions on the kinds of messages that could be written down. In practice, a great number of abstract meanings and meaning elements could not be symbolized. However, when the writing systems gradually developed from being more or less completely content-based to being expressionbased instead (signs or letters were used to indicate the sounds of speech quantized as syllables, vowels and consonants), these restrictions were removed. In principle, the segmental aspects of any string of spoken behavior could be satisfactorily symbolized in writing, and this established a "formal autonomy" of the written text.

But even after the invention of the Greek and Latin alphabets there were quite strong restrictions on both the form and content of written texts. One reason for this was that much of what was written down in the beginning consisted of important parts of the already existing oral tradition: epics, verse, song, orations used as laws, folk tales, legends as well as proverbs, adages, aphorisms, riddles, etc. These texts were biased both in form and context to fit the requirements of oral communication and auditory memory. Thus, they often had a formally bound 
structure (e.g. verse), they were typically elliptical and left a lot of the interpretation implicit. For their interpretation, they were heavily dependent on the interpreter's background knowledge of the culture and of the referents involved, and the functions of the various texts in the entire cultural context, etc. Thus, they tended not to say exactly what they meant; they were not "semantically autonomous" in Olson's terminology.

Before printing was invented, every text had to be laboriously copied by hand each time it was to be duplicated. Of course, this also put the writer under strong pressure to keep texts short, and hence the texts were not very explicit. The situation was radically altered, when printing made it possible to spread a very large number of copies of the same text. It was by this means possible to increase the length of texts and to write in a much more explicit manner. Many more background assumptions could be expressed if necessary, and this, according to Olson, made the written text "semantically autonomous".

Olson points out that soon after the advent of printing, Martin Luther pronounced his opinion that the meaning of the Scripture depended, not upon the dogmas of the church, but upon a deeper reading of the text. This is, in other words, the theory that the meaning of the text is in the text itself. Olson traces this theory back to Luther and to the British essayists of the seventeenth century, who were among the first to try to pursue the ideal in writing of explicitly formulating all the steps in the writer's argumentations without any reliance on implicit presuppositions or personal interpretations. This ideal later on motivated the rectification of natural language into a language of $\operatorname{logic}$, which was supposed to meet these requirements better than normal prose. Olson seems to accept the thesis of the semantic autonomy of (certain) written texts; meaning is said to be "intrinsic to language" in a written message but "extrinsic" in spoken communication. Such a view is hardly acceptable, however. The interpretation and comprehension also of a written text crucially involves background knowledge which is not contained in the text as such (VII.7). Of course, it remains true that a 
written text is in general more explicit and more autonomous than a spoken message (II).

With the invention of writing it became possible to retain messages over centuries, something which had an enormous impact on the development of human cultures through the accumulation and maintenance of the insights of earlier generations. However, literacy had many other important psychological and social consequences for individuals and social groups some of which will be briefly reviewed here (see Goody 1977).

Many of the very earliest written documents were of an administrative or economic nature; inventory lists, catalogues, and lists of taxes, credits and debts etc. Other types of texts that appeared in early times were laws, decrees and instructions of various kinds. The use of such specializations of writing as lists, tables, diagrams and formulas is particularly interesting in that some of them exploit the spatial dimension of the written surface in a way which has no counterpart in speech behavior. The user thus gets an opportunity to manipulate his subject matter in new and important ways. Logical and mathematical calculations become possible. Such arithmetic operations as multiplication and, in particular, divison are hard, if not impossible, to carry out without written notations. Moreover, could the position system of arithmetics have been invented without writing?

A written text is independent of its producer and interpreter in a way that has no counterpart in the case of spoken messages. This enables the user to keep a certain distance to the contents, as well as to his communication partners. Speech communication, on the other hand, is firmly anchored in the immediate

1) It should be noted that these hypotheses about the cognitive consequences of literacy (cf. below) apply primarily to the historical development of cultures. It is probable that there are some similar differences in cognitive and social attitudes between literate and non-literate individuals in contemporaneous society, but, as Scribner \& Cole (1981) point out, one cannot leap to the conclusion that what was necessary historically is necessary also for children, who are born into a society in which elaborate uses of literacy have existed for a long time and thus presumably have had repercussions on general modes of thinking (i.e. even among nonliterate members). 
environment, the speech situation which is embedded in the everyday culture, all of which exerts a strongly normalizing pressure on both speaker and listener. They have to follow the established conventions of their culture, unless they deliberately accept confrontation with the ensuing negative reactions from the social environment, thus taking the risk of being expelled from the community in the long run. The written medium, however, greatly extends and enhances the user's chances of taking an independent personal stand with respect both to the contents of messages and to his fellow human beings. This will have several important consequences.

In a literate culture attitudes towards reality are changed. Some features of the down-traded picture of everyday reality can be questioned, alternatives can be considered and compared, and rationality, skepticism, logics and science developed - all of which is commonly associated with Western culture. Knowledge tends to be more abstract and general, and less tied to the contexts of everyday reality. The development of logic, for example, has to do with the relative freedom to manipulate the elements of written texts; the written medium allows us to separate words, which means that their internal ordering can be more easily manipulated, and that syllogistic reasonings can be developed. The use of tables makes it easier to construct classificatory systems with hierarchically organized categories and subcategories. Writing allows the user to work out and survey all of the theoretically possible alternatives.

Communication by means of written texts puts the emphasis on the intellectual, cognitive, descriptive, and argumentative functions of language, whereas the emotive and social aspects are kept firmly in the background. After all, most of these latter aspects are conveyed in speech by means of prosodic phenomena, accents and intonations etc, and various non-verbal signals and gestures, and this is all very poorly represented in writing. Mcluhan (1965:86) has argued that the use of alphabetic writing as a medium of communciation has made Western civilized man into an intellectualized individual who represses his feelings and his commitment. 
Writing helps its user to sharpen his thinking; language is thereby given a more individualized, monologic function. This in turn facilitates the development of intellectual independence on the part of individual persons. While deviant opinions and attitudes are difficult to convey and maintain in a society where communication is based on direct face-to-face interaction, the literate culture enhances individualism; heretics, free-thinkers and philosophers are given a certain amount of elbow-room. Single historical individuals can now make their own original contributions to the development of knowledge and culture.

In this context we must reiterate that writing implies that knowledge can be accumulated over a period of time. There is no longer any need for each generation to repeat the same process of trial and error over and over again, since one is no longer forced to rely only on the oral tradition, which is, after all, limited in scope.

Another consequence of the rise of literate culture is the possibility for men in power to control secondary groups. By issuing written decrees and edicts the desires and orders of a central power can be distributed to large groups of people living at different places. This presupposes and carries with it the development of an impersonal bureaucracy. The possibilities of governing and dominating people with the help of written messages are of course particularly great in societies, where literacy is restricted to a small minority, and this is precisely the way in which almost all societies have been governed up to our own time. This also means that those who are competent to use the written language enjoy a high social status, and the written language itself is normally regarded as the correct language (III.3). Thus, language in a literate society, or rather a society with varying degrees of literacy, becomes a potent means of social stratification. The "restricted codes" of spoken language are insufficient as a basis for societal advancement and career. Washabaugh argues:

"Nonautonomous, restricted codes are poor social integrators since they point speakers to no authoritative group or tradition (Mueller 1971:111). Nonautonomous, restricted codes 
increase a people's commitment, to, and dependence on, the social order and lead them to support a repressive social order all because such a language form decreases a people's ability to reflect on their condition and to generate 'privatized' alternatives to the social order (Mueller 1971:106)."

(Washabaugh 1980:211)

It has also been pointed out that literacy makes the writing of a (relatively) objective history possible. Nonliterate cultures are characterized by the existence and vitality of myths, both religious and secular. Every culture feels a need of explanations of why nature and society are the way they are. Myths explicate and legitimize the existing societal conditions, and they normal$1 y$ do so in a way that satisfies the interests of those in power. Though myths generally build upon a kernel of historical truths, their contents constantly change through oral tradition as society develops and as society's demand for new types of explanations and legitimizations are altered. When written records are available and can be preserved over a period of time, one of the preconditions for making objective history possible has been created.

Finally, the invention of writing has naturally had important consequences for the linguistic code 1 tself. In most literate societies we will, sooner or later, be faced with two codes or variants - one is tempted to say two languages - rather than just one. The written language is different from the spoken language not only as regards the medium of representation graphic as opposed to phonetic. Its grammar, particularly its syntax, is much more constrained and prescriptively controlled, and its lexicon is greatly expanded. When written dictionaries and encyclopedia are being developed and used, the size of the total vocabulary of the language involved is very much increased, and as a consequence the delicacy and precision of word meanings are also enhanced. of course, these gains achieved through written language will have important feedback effects on the spoken language too. (It is hardly a coincidence that sign languages (i.e., those languages used by the deaf) have con- 
siderably smaller vocabularies than most spoken languages. There may be several reasons for this state of affairs, but the fact that spoken languages are supported by their written counterparts (whilesign languages have no such counterparts /at least not until some recent attempts to remedy this situation, cf Stokoe 1972, Baron 1981/) seems to be a major factor. The gestural medium as such seems to have an inherent capacity for symbolization (reference and description) which is just as large as that of the vocal medium).

By way of conclusion we may say that the development of written language and literacy is no insignificant matter. On the contrary, it has far-reaching consequences for cultures, which become differentiated and specialized, for individuals, whose attitudes to knowledge and society are radically changed, and for the linguistic codes themselves, which are transformed in certain respects. Therefore, the investigation of the differences between spoken and written language is worth pursuing in linguistics too.

\section{III.2 The traditional tasks of linguistics}

In today's highly industrialized and diversified western societies many states have been able to use some of the economic surplus in order to hire numerous professional specialists who can pursue highly "theoretical studies" with the aim of finding out the true nature of various matters, independently of immediate practical, political or ideological goals. Thus, the field of theoretical linguistics studies general conditions of the structures and uses of natural languages. (At least, this is what we think we are doing.) However, this is something very new. Traditionally, the tasks of linguists have been more or less directly related to practical enterprises and political goals, and this is of course still true of a great many linguists presently working in many countries.

Traditionally, linguists have worked on tasks concerning the development of written standard languages (and this of course 
presupposes a sufficiently developed theory of the structures of the corresponding spoken languages), the promotion of literacy, and the improvement of the study and teaching of foreign languages. However, these activities have more often than not only been the means for attaining higher goals, especially that of serving the right religion and its various institutions and that of serving authorities of a more secular kind by establishing a language for governing and controlling people.

To a very great extent the history of linguistics is connected with the activities of religious institutions. In fact, linguists have most often been priests with the important tasks of taking care of God's holy word, preserving and explaining it, providing the correct interpretation of the scriptures and maintaining its proper form, and translating it to new languages, thereby making their contribution to the spread of the right religion to pagans and barbarians.

Linguists were priests during the era of ancient Indian linguistics, their task being to preserve certain ritual and religious, orally transmitted texts. The history of Western linguistics is very closely connected with Christian theology. This is true of the entire Middle Ages from St Augustine onwards. Hovdhaugen notes $(1980: 129)$ that "Christian theology is to a large extent exegesis of the Holy Scriptures, and exegesis is to a large extent a linguistic analysis".

Linguistics in Classical Greece and Rome was in certain respects different, since grammar was at that time more directed towards the explication of the (written) language of the great authors. The same is therefore also true of the humanists of the Renaissance. One should note that this very much involved the study of foreign languages. Homer's Greek was a foreign language for most scholars in the Hellenistic world, and classical written Latin was a foreign language throughout the Middle Ages, and of course later on too. 
The connection between linguistics, or philology, and the church was also reflected in the school system of most Western countries. Language pedagogics was built upon classical and medieval linguistics, especially grammar, but also rhetorics, and logic or dialectics. The aims of the grammar schools were twofold: to instruct the pupils in the right faith, and to provide them with the correct language, i.e., Latin and (later on) the written standard national language (but not of course their own vernacular which was considered faulty and vulgar). These two tasks were united, and could not and must not be disconnected.

Even our 20th century history provides examples of the connection between religious aims and linguistics. American descriptive linguistics has largely been concerned with inventing alphabets for, and discovering the grammatical structures of, numerous American Indian languages. The ultimate goal has usually been the translation of the Bible and the integration of Indians into the society of White Americans.

In modern times linguistics has served more secular rulers. Among the most important tasks we find language planning and language cultivation (and the ancillary disciplines that might be needed for these activities); the goal was (or is) to create standard languages for new nations, i.e., to develop and establish a language variant suited for administration, government and control, and for the development of a national literature. In practice, this means the standardization of a language, more exactly a written language, most often the language of the politically and economically dominant groups or tribes. Again we see linguists concerned with the establishment and legitimization of a correct language, that which should be the norm for the citizens. To some extent this may have served the imperialist goals of social repression:

"In this context recall the remark made by Elio Antonio de Nebrija, the first grammarian of the Spanish language. The year was 1492 and he had just presented the Spanish queen 
with the first copy of his Gramatica castellana. Her question to him was potentially disarming: What good is your grammar? What do you do with it? Fortunately for grammarians everywhere, Nebrija had just the right answer. You need it, he explained, to assure that all peoples subjected to the Spanish crown will have one voice with which to talk to the Queen. Besides, he continued, 'siempre la lengua fue compañera del imperio' -- language has always been the companion of empire."

(Di Pietro 1976)

As I pointed out at the outset, the economic surplus, which has been produced in some industrialized countries in the last century, has to some extent liberated some scholars from the immediate practical and political demands and goals which are normally set by society. We can, e.g., afford to have a limited number of "theoretical" linguists. In the same period we have observed a clear trend on the part of linguists to pay more attention to spoken language. In fact, it is part of today's standard textbook ideology to assume that spoken language is the primary and most important medium of natural language (V). Surely it can not be denied that linguists have invested a lot of energy and ingenuity in the study of spoken language. Some well-established fields are dialectology, phonetics and anthropological linguistics. In recent years we have witnessed a formidable explosion of studies in discourse analysis, pragmatics of spoken language, and related areas.

Thus, the written language bias in linguistics now seems to be diminishing. The very fact that I hit upon the idea of writing this book is a small but perhaps significant symptom of precisely this trend. However, traditions are not so easy to change. My aim is therefore to invoke some discussion around questions like the following: To what extent is there still a written language bias in linguistics? In what ways must linguistics be modified in order for it to tackle the problems of spoken language in the best possible ways? 


\section{III.3 The status of written language}

In most (all?) societies the written language has had, and still has, a very high status. It is regarded by the common man with respect, admiration and reverence. Originally, it was the language of religious documents, the laws (both religious and secular), and the great authors.

of course, the traditional admiration for the written language is basically an admiration for those, the very few, who could use it properly. One should recall that in most societies there has been, and still is, no wide-spread literacy. Instead we have had oligoliteracy, i.e. a state in which literacy is limited to a small minority of educated people. No wonder writing is regarded as something special. Already among the sumerians and Accadians, "writing was the pursuit of scribes and preserved as a 'mystery', a 'secret treasure'" (Goody \& Watt 1972:323) and this is still true of certain societies, e.g., in the Middle East.

Thus, the supremacy and mystery of the written language were established in societies with a low degree of literacy. Furthermore, the association with the church and its institutions added to its status. For a long time, priests and monks were a very important group among those who were able to read and write, and they also acted as school teachers and private tutors, and this gave them further opportunities to enhance the specific status of the written language. This is not to say that an admiration for the written language as a medium is unmotivated. In fact, literacy enables people to perform many important cognitive and social actions, which would be impossible without writing (cf III.1).

We noted in $\S$ III.2 that nationalism and central state authorities may consolidate their power and strength by means of the written medium and its standardization. However, we must be aware that standardization is a natural and inherent feature 
of any attempt to "convert speech into writing". No writing system can preserve all the variation present in natural speech. The crucial point is therefore not standardization as such but the level of abstractness at which the standarization of the written notation is established. In principle, the more heterogeneous the dialects one tries to cover by one common written stanclard are, the more remote will this written code be relative to the individual spoken vernaculars (and idiolects) (except possibly the dialect of the men in power, which is usually the basis of the norm). And in most parts of Europe there were indeed many often mutually very different linguistic varieties, which - as part of the formation of the new national states - were transformed into "dialects of the same language" precisely through the establishment of a common superordinate written norm. In other words, this written norm came to constitute a separate "language" (e.g. German, Italian, Swedish, Russian), i.e. that language of which the original spoken varieties were to be regarded as "dialects". Thus, by creating national written standard languages centralists strengthened their power and tried to impose a national identity onto a conglomerate of different tribes and ethnic groups, who had so far, in many cases, not had very much in common ethnically and/or linguistically. At the same time, this process served to reinforce further the idea of the written standard language as a superior linguistic medium.

The high status of written language still prevails in today's highly literate and secular Western industrialized societies. The written standard language is the norm; it defines what people generally consider to be linguistically correct. The written word carries with it permanence, firmness, responsibility; only written statements are ultimately valid as legal documents and in judicial procedures. In fact, the written language belongs to the very foundation of the society and the national state (compare the function of national languages).

One of the most common ingredients in laymen's outlook on language is the view that written language is the proper language, whereas the spoken vernaculars are crude, primitive, incorrect, 
and unsuited for higher functions. This view is traded down in school teaching. After all, the main task of the primary school is traditionally that of teaching children how to read and write, and how to use language properly, i.e. not as it is used in normal casual speech. It is obvious that schools in western societies have communicated this view of written and spoken languages in surprisingly similar ways from antiquity, through the Middle Ages and further on right up to the 19 th and 20 th centuries, and it is debatable whether they are actually changing to a more moderate position today. ${ }^{2)}$ I have already alluded to the close connection between the traditional tasks of school education, the promotion of the right faith, and the study of the correct language. For a long time, the objects of study were the Holy Scripture and the great authors, and the analysis usually focused on formal aspects, especially the grammar, of the written varieties used there. Originally, the language studied was Latin, but the same attitudes and procedures were later transferred to the study of the national standard languages. The grammar of Latin was used as a model for the writing of the grammars of the various national languages, and these languages were analyzed and taught in much the same ways. In fact, the variants of the national languages to be used in religious practice, education, administration, and legislation were largely shaped, even invented or created, on the basis of Latin. (In Scandinavian countries, German partly played the role of an intermediary language). The deep-rooted attitude towards the mother tongues of the pupils has survived for centuries and millennia; the pupil comes to school without having a proper language! Later we shall study how such attitudes are reflected in contemporary linguistics (XI.2).

2) I am only familiar with Scandinavian school systems in this regard, but I assume that most Western countries fit the same mould. The history and traditions of the teaching of Danish and Swedish in our countries have been throughly described by Diderichsen (1968) and Thavenius (1981) respectively. 
Thus, we have pointed out the position of the written standard language and the spoken vernacular as high vs low status languages in the layman's conception, in what could be called the "linguistic folklore" or "ethnic linguistics" attitudes are found among professional linguists, and this is hardly surprising. On the one hand. linguists are of course members of the society at large, and the adoption of the unquestioned attitudes of the culture is part of the socialization of those who later become linguists just as much as it is part of other people's upbringing. Secondly, the activities of the linguists themselves have served, confirmed and enhanced the very same attitudes and conceptions. We noted earlier (III.2) that linguists worked in the service of God and the king not only in describing and analyzing but also in cultivating, developing and establishing the structure and use of the proper written standards. Linguistic behavior is conventional, it is subject to rules and norms of various kinds, and since the written language was regarded as the correct language (language as it should be), that which should be aimed at and established, it was only natural that grammarians concentrated on trying to find out the rules underlying that variant. In today's structural linguistics we can sometimes see how issues of gramaticality are solved by (implicit) reference to the inherited norms of written language $(\mathrm{V}, 8)$.

3) It must be admitted, however, that we do not yet have much systematic knowledge of the linguistic folklore and its functions. Apart from some sociolinguistic studies of isolated points, there seem to be few or no studies of this interesting topic. What we know is mainly what can be gleaned from subjective impressions of how laymen tend to talk about language in everyday conversations, how their opinions surface in e.g. the letters-to-the-editor columns of daily newspapers and magazines, how teachers in elementary schools behave with respect to language, and what school books have to say about language. 


\section{III.4 Written language as metalanguage}

The bias towards written language has been forced upon linguistics for two different reasons or at two different levels. on the one hand, our object of study has traditionally been the written language, and the reasons for this have just been outlined. On the other hand, the written language also serves as metalanguage, as the language of linguistic description. When we analyze language and linguistic phenomena (be they written or spoken), the analysis is performed by the use of written languages, and the various concepts, theories and results of analysis are formulated in that medium (plus pictures, diagrams, lists etc, but these are also writing in a broad sense). We may therefore suspect that our concepts and theories may be dependent on the inherent limitations and possibilities of the metalanguage. Even if we are in fact analyzing spoken language, we have to represent that language and the corresponding verbal behavior, and this we do by means of common writing lor some slightly modified variant of it, such as phonetic transcription or a symbolic notation like that of formal logic). This means that our data are transformed into written language, or at least into something that is heavily dependent on written language. I will discuss some of the consequences of this below le.g. as regards phonology and the theory of communication). ${ }^{4}$ )

4) If we narrow down our perspective a little, we may recall the unusually important role ascribed by transformational gramnarians to the notational systems used in linguistic models. The form of the theoretical apparatus is considered to be of utmost importance, and the form is that which is provided by the meta-language (e.g. in Chomsky \& Halle 1968). "There has been a remarkable tendency for even the most eminent transformation grammarians to pay more attention to the typographical combinatorics of their notational systems than to the content that is expressed in them and to condone even drastic innovations as long as they stay within or close to familiar notational systems" (McCawley 1980:919). 
It should be noted in passing that today's linguistics is beginning to use computers quite extensively, and we have already seen how theories, models and metaphors from the computer sciences are coming into linguistics. It would be worth while considering how much of these new conceptions depend on the inherent properties of digital computers (cf. e.g. VII.I0 on the representation of knowledge).

Naturally, the two functions of written language within linguistics, the functions of being both object of study and language of description, interact in complex ways and thus add up to a state of affairs, in which it may be difficult to isolate the contributions of the one or the other. 
IV. THE WRITTEN LANGUAGE BIAS IN LINGUISTICS: ON THE SCOPE OF OUR CLAIMS

I am going to argue that contemporary linguistics is rather heavily biased due to the tradition of studying mainly written language. Since it is quite obvious that my claims can easily be misunderstood and misrepresented, I would like to try in the next few pages to define more precisely the scope of my claims and to express a few provisoes before starting on the real subject matter.

First, I would certainly not claim that spoken language and written language are completely different in nature. In many respects the differences are clearly gradual rather than absolute. The structure and use of written language is of course largely derived from the properties of spoken language. This holds at two levels, both that which consists of the whole culture (and here we must again discern several layers, e.g. the national culture defined by the existence of a certain national standard language, and the whole civilization of the Western world $\left.\mathrm{d}^{4}\right)$, and that which is constituted by the evolution of the single individual who first acquires speech and later on learns to read and write. Hence written language may partly be characterized in terms of the same fundamental properties of natural language and the same conditions on human communication, perception, cognition etc. as spoken language. Most of what occurs in standard written languages may be said to represent a rectification of linguistic structures which show up in similar forms in spoken language.

But, on the other hand, I do mean that the differences between the two media have important consequences. If the structure of written language is a rectification of that of spoken language, then this rectification involves some important changes; some

4) I have nothing to say here about languages and linguistics in other cultures, e.g. in the Far East. 
aspects of language are enhanced and emphasized, whereas others are attenuated or eliminated. Communication by means of written texts, pictures, diagrams etc. are subject to other conditions than speech communication (cf. II). Thus, for example, we can organize and survey much more material in an orderly manner. Writing provides the user, the individual as well as the collective, with new means of communicating, thinking, performing cognitive operations, and executing social control, and it changes the attitudes towards social groups, towards knowledge of different kinds, and towaras language itself. The importance of all this must not be belittled (III.1).

Second, I will certainly not claim that all those concepts which have been developed by linguists in their analyses of written language are inadequate when applied to the structure of spoken language. Again we are often faced with matters of degree. Many concepts are more adequate and suitable for the analysis of written language, because they emphasize aspects which are most evident in (certain variants of) written language. A modest claim would be to say simply that many of the views and concepts to be discussed in this book are naturally at hand for someone who has been accustomed to analyzing written texts rather than speech. But even that is not uninteresting.

on the other hand, some of the concepts derived from written language are indeed thoroughly misconceived as applied to spoken language. (Some of these are in fact rather inadequate for written language too, but they are at least not as counterintuitive there). The moral is therefore that we have to discuss the consequences of the theoretical proposals, whether to abandon them or not, in the specific cases one at a time.

Furthermore, I am not going to claim that the tradition of anaIyzing written texts is the only reason why linguistic theory is the way it is. In some cases the ultimate or original causes and reasons should be sought elsewhere. Some of the features which are in general associated with written texts, may occasion- 
ally occur in specialized variants of spoken language. For example, this applied to the high degree of conventionality which applies to form and content in certain ritualized forms of speech (e.g. verse, orations, proverbs etc.). Such phenomena occur also in illiterate cultures; in fact they were among the first things to be written down, when writing was invented (c.f. III.1). Thus, we must of course admit that the specific features of written language did not arise out of nothing. They are developed out of specific ways of speaking. On the other hand, it must be reemphasized that writing, once it exists, will necessarily be further developed, and it will then enhance certain aspects of language while others will be tuned down.

A more general point related to the one just raised should be made in this context. In this book I frequently stress the fact that the written medium makes a focus on the products of behavior natural and indeed necessary, while speech should require an analysis that accounts for its character of a dynamic, interactive and time-distributed stream of activities. However, there is some natural basis for a focus on products also with regard to spoken communication as such. It is generally agreed that no one can become aware of the biological or mental machinery involved in complex motoric or cognitive processes, thinking as well as production and perception of utterances (e.g. Nisbett \& Decamp Wilson 1977). Awareness, whether based on introspection or on observation of overt behavior, seems to be restricted to the outcome or results of the mental processes. Therefore, we find here a basis for our tendency to analyze and explain thinking with regard to its intentional objects rather than the thought processes themselves (cf. VII:9). Furthermore, it may be that the visual mode is generally stronger in imagery than any other sense modality, including the auditive one. All this seems to make provisions for a deep-seated focus on visually encoded products of mental processes. Nevertheless, I would reiterate what I stated above; writing promotes a strong cultural reinforcement and consolidation of those tendencies, despite the possibility that the same tendencies may have a certain 
natural basis in the human constitution.

Finally, a secondary remark; some commentators seem to assume a negative attitude on my part towards written language and its linguistic analysis. Nothing could be further from the truth! No one can deny that writing is a wonderful resource which enables cultural man to perform innumerable new and extraordinari1y important things. Therefore, the study of written language, and the production and development of written language as well as the reading, comprehension and interpretation of written texts, is very much motivated, necessary and interesting. At the same time, however, it must be said that linguists (and other scholars) have worked much too little with spoken language (much less than we think we have), and have not sufficiently probed the question of what it really means to analyze speech in its own terms, i.e. as a temporally distributed, dynamic behavior. It seems to me that it may be useful - as part of an attempt to remedy this - to explore the extent to which our current conceptions of language can be explained and understood within the perspective that I will sketch, i.e. the written language bias. 
V. LINGUISTICS AND THE OVERALL THEORY OF LANGUAGE

"La tâche de la linguistique sera:

a) $\ldots$...

b) $\ldots .$.

c) de se délimiter et de se définir elle-même."

(Saussure 1964:20)

\section{V.1. Linguistics and other language sciences}

It seems appropriate to start our survey of trends in traditional and present-day linguistics by asking how linguists tend to define (explicitly or implicitly) the scope of their discipline. 'Linguistics' is a term which covers certain types of scientific approaches to language; it does not denote all sciences or scientific activities that are concerned with language and the use of language. On the contrary, both insiders and outsiders would identify linguistics as a humanistic discipline which analyzes language only in some specific ways and respects. " Two historical factors are responsible for this state of affairs. First, there has been a time-honored scholar1y tradition directed towards the study of certain aspects of language. This tradition used to be called an art, but today the designation science is more fashionable. Secondly, this tradition has been institutionalized in the traditional academic systems of the Western countries, which means that there are special departments of linguistics and the linguistic study

1) The term linguistics borrowed from English (and/or from French linguistique) is nowadays used in most other languages too (e.g. German Linguistik, Russian lingvistika, Swedish lingvistik), but many of these languages also use native terms like Sprachwissenschaft, jazykoznanije, sprăkvetenskap, Dutch Taalwetenschap, Italian glottologia, etc. to denote the same traditional humanistic study of language. Sometimes a distinction is made between linguistics proper and philology. Both, however, belong to the linguistic tradition with which I will be concerned here, although I will mainly deal with linguistics proper. 
of specific languages. ${ }^{2}$

What then is the traditional content of linguistics? originally, i.e. through most of our long history almost up to the 19 th and 20 th centuries, it contained phonology, grammar (especially morphology and syntax (which, however, did not consider word order very much) and some of their semantic aspects), logic, and rhetorics, and also philology (text interpretation etc.). Later, philology was (temporarily?) relegated from linguistics (because it did not study language for its own sake), and something similar happened to logic and rhetorics. Hence, according to our 20th century conception, linguistics is basically phonology, grammar (morphology, syntax) and semantics (especially word semantics; sentence semantics is a rather recent rediscovery). In the 1970's logic and rhetorics have also been reincorporated under the headings of (formal) semantics and pragmatics, respectively.

In a more global perspective, the linguists' approach(es) to language must be considered narrow, and this is also true of today's linguistics. For example, one very important aspect of language which is seldom or never discussed in linguistics (and this also applies to psycho-linguistics) is the question why people use language, for what purposes utterances and written messages are compiled and used (cf. §VII.1 on the why of communication). Instead the typical linguist's attitude is this: Given that people use messages formulated in natural languages, i.e. in terms of linguistic expressions, how are these expressions structurally organized, and what meanings can be attached to the expressions qua items in an abstract, supraindividual

2) Here the term linguistics (or its equivalents, cf. fn. 1) would cover the activities both in general linguistics and in the linguistics of specific languages, e.g. English linguistics, Romance linguistics, Uto-Aztec linguistics, regardless of how these studies are organized in academic institutions in various countries. 
Iinguistic code ( $=1$ a langue, of $\S \mathrm{V} .2)$. Accordingly, linguistics in the usual traditional (though still widely accepted) sense is narrow in scope, (Lyons, 1981:36, has proposed the term 'microlinguistics' for it). Many scientific studies of the structure, function and use of language simply do not belong to linguistics in this traditional sense. Such fields as the psychology, sociology and philosophy of language are concerned with linguistic phenomena, but they are said to do so from the vantage points of other disciplines (outside linguistics). Such scholars as Wundt, Mead and Wittgenstein do not belong to the history of linguistics, although they made contributions to the study of language which compete quite well with those of, say, Humboldt, Sapir, Saussure and Chomsky. Even such borderline disciplines ("Bindestreichlinguistiken") as psycholinguistics, sociolinguistics and neurolinguistics do not really count as linguistics proper, according to many scholars in the field. (They would, however, be included in a more comprehensive concept of linguistics, Lyons' (op. cit.) 'macrolinguistics', which is now gaining acceptance, cf. below).

Linguistics has traditionally been concerned with written language, i.e. the language of the Holy Scriptures, the great authors, and later on simply with what is considered to be "good" written standrads, educated or "received" English, German (German Hochsprache), Swedish, etc. Furthermore, the focus has been on formal rather than semantic aspects. In fact, grammar has for centuries been the kernel of "real linguistics".

The concentration on grammar was quite natural as long as linguistics served the practical goals set up by the church and its allies, the educational system and the national state (of. III.2). At school, foreign languages were taught - at first Latin and later on other modern languages (in Sweden German, French and English) - and in this context grammar played an important role, especially so because the means for acquiring knowledge of the foreign languages was always the study of written texts. The study of the mother tongue was also complete- 
Iy focussed on the written language, and it does not seem entire1y wrong to say that the written standard taught at school was also something of a foreign language for the pupil coming to school equipped only with his spoken vernacular. At any rate, learning to write correctly involves in no small measure learning to follow a number of grammatical rules which do not necessarily hold for the spoken language (cf. VI:3).

But in today's theoretical linguistics, too, grammar theory indisputably constitutes the kernel of linguistics proper. Some linguists would even argue that 1 inguistic theory equals grammar theory. This is, for exampple, not far from the position actually held by most generativists. It is true that the term 'grammar' is nowadays used in a rather comprehensive sense. Traditionally, 'grammar' meant morphology and syntax (the latter comprised mainIy the study of the grammatical functions of words and phrases), whereas today the term is often used to cover the entire "language structure" including phonology and semantics (though the focus is still more on the form that the content).

Thus, there are historical reasons why linguistics developed into a rather narrow, abstract discipline treating certain aspects of language structure as if they were autonomous (and I will have still more to say about this below). We still live by this conception of linguistics, aptly characterized by Derwing (1973) and others as 'autonomous linguistics'. Yet, it should be outdated by now, incapable as it is of providing a real understanding of the nature and functions of language. ${ }^{3)}$ Language is em-

3) I have ignored here other attempts to justify an autonomous linguistics. For example, many linguistics since Saussure, not in the least Hjelmslev and Chomsky, have wanted to consolidate Iinguistics as an 'immanent' discipline independent of psychology, physiology etc. It is true that chomsky le.g. 1968:84) argues that Iinguistics should be regarded as a subdiscipline of cognitive psychology, but his actual theories and methodological recommendations go very much against his. In fact, the intense and rather devastating critique that has appeared in the 1970's, demonstrates the failure of Chomskyan autonomous linguistics as regards its claim for psychological validity (e.g. Derwing 1973, Linell 1979a, Botha 1979). 
bedded in acts of communication and cannot be properly described and understood out of communicative contexts. This holds a fortiori if we want to understand the nature of spoken language. This means that relevant parts of psychology, sociology, anthropology, ethology, neurophysiology, etc., must be taken into consideration; therefore, many scholars in the field (including myself) would now hold that the Bindestreichlinguistiken should be recognized as linguistics tout court. Fortunately, there are right now strong currents leading in this direction; linguistics would then become a cover term for those scientific approaches which deal with human natural language(s). ${ }^{4}$ Such a linguistics would be characterized by methodological pluralism; it should study all significant aspects of language and its use, including its relations to other types of symbol systems and other types of behavior.

\section{V.2. Structure and use: Language vs parole, competence vs performance}

"Writing cannot be flatly dismissed as an imperfect, conservative quasi-transcription, as has often been done up to the present day" (Vachek 1949:93)

We noted in Section IV that speech and writing are largely based on the same underlying language system and that many categories of analysis should or must be the same for both types of manifestation. But I also pointed out that there are likewise a number of important differences, and it must be an empirical task to find out how far-reaching these are. Obviously, there are considerable culture-specific differences; the spoken and written variants of chinese are clearly much more different from each other than speech and writing in, e.g. English. However, I cannot here and now go into the problem

4) Cf. Lyons (1981:36) 'macrolinguistics' (see above). 
of what the actual structural relations between speech and writing are. Instead I wish to call attention to the ways in which modern linguistics tends to treat the relationships between the structure and use of language in speech and writing.

If we have to make a very succint statement, we should say that linguists tend to ignore the differences between the language systems underlying speech vs writing. It is assumed that there is one single system, what Saussure termed la langue (e.g. the English language = the langue of the English-speaking culture), which underlies both kinds of manifestations. In Chomsky's terms we would assume one monolithic linguistic competence, not an aggregate of partially distinct capabilities, e.g. one for language used in speaking and listening, and one for language used in writing and reading.

However, in addition, the manifestations of language in speech and writing are assumed to exhibit great similarities. Furthermore, 'it is one of the cardinal principles of modern linguistics that spoken language is more basic than written language' (Lyons 1981:11). Writing is usually treated as if it were merely a secondary manifestation, a relatively unproblematic and theoretically uncontroversial reflection of spoken language. Such a view has been held for long; it was emphatically pronounced by e.g. J.J. Rousseau:

\footnotetext{
'Les langues sont faites pour être parlées, l'écriture ne sert que de supplément à la parole ... I'écriture n'est que la représentation de la parole, il est bizarre qu'on donne plus du soin à déterminer l'image que l'objet.'
}

(Rousseau quoted by Derrida 1962:42,54)

Most modern linguists espouse similar views. Thus, Hockett, in his influential text-book (1958), makes only one distinction, that between "language" and "writing" (p.4), and it is quite clear that "language" here means spoken language; "language excludes writing" (op cit:11). Turning to the founders of structuralist linguistics, we find statements like the following: 
'Language et éctriture sont deux systèmes de signes distinct l'unique raison d'etre du second est de représenter le premier'

(Saussure 1964:45)

'Writing is not language, but merely a way of recording language by means of visible marks'

(Bloomfield 1933:21)

'For the linguist, writing is, except for certain matters of detail, merely an external device, like the use of the phonograph, which happens to preserve for our observation some features of the speech of past times'

(Bloomfield 1933:282)

Apart from this, the prevailing attitude implies that the actual use of language is linguistically uninteresting, and that linguistics must be concerned with the theory of the underlying system la langue) or the ideal speaker-listener's competence, not with the preformance of actual language users. Note, however, that when performance is referred to, it almost always is the performance of speech communication that is presupppsed. Saussure simply used the term parole (literally: 'speech'). Such an attitude is largely unjustified. Although in general speech takes precedence over writing structurally, historically and ontogenetically, there is no doubt that writing is a medium with partly quite different inherent possibilities and functions (see III.1). Therefore, it is simply not true that writing is "merely an external device" used to "preserve for our observation some features of the speech of past times". I will come back to this issue presently.

We now come to what may be seen as a remarkable paradox, viz. the obvious contradiction between what modern linguists say about the relations between speech and writing, and what they actually do in practice. On the one hand, linguists maintain that speech is primary and, therefore, the most (or only) interesting object of study. On the other hand, their actual practice reveals quite different traditions and values. To a very great extent, linguists still study written sentences 
(often out of contexts) and written texts, and to the extent that spoken language is actually studied it is done with a conceptual apparatus which is more or less totally derived from the experience of written language analysis.

Returning to Rousseau, we note that he protested rather vigorously against the linguists' one-sided interest in written language. He complained that:

'Pour les Grammariens, l'art de la parole n'est presque que l'art de l'écriture'

(quoted in Derrida 1962:56)

Saussure used quite strong words about what he called the "tyranny of letters" (1962:53) which he considered to be a moral perversion ("un fait pathologique") and a sin of idolatry ("un péché d'idolatrie"). Yet, the traditional practice and attitudes of linguists have stayed on. It is true that the primacy of speech is nowadays verbally acknowledged, but the ways in which linguists construe their theories and seek their applications have not been sufficiently revised. Accordingly, the distinctive characters of spoken vs written communication are poorly understood. Therefore, what is needed today is not only a more adequate analysis of spoken language but also a general recognition of the independent importance of writing. (The latter is something which Derrida $(1967 a, b)$ wants to achieve in his so-called grammatology.)

There are obvious differences between speech as opposed to writing, spoken language as opposed to written language, communication via spoken utterances as opposed to written texts, all of which have been insufficiently attended to and inadequately accounted for in contemporary linguistics. It is most noteworthy that there seem to be some differences not only in the performance but also in the language systems underlying speech vs writing. That is, spoken and written language have partly different standards of their own ${ }^{5)}$. For one thing, the substan-

5) In modern linguistics, this has been seriously discussed only by very few scholars, e.g. Vachek (1939, 1949). 
tial content of the linguistic rules is not quite the same for the two media. Secondly, the impact of norms seems to be different. In the dynamic interaction of spoken discourse, where rapid responses are legion, grammatical norms seem to play a somewhat subordinate role, whereas in written discourse the conventional and explicit rules exert a considerable influence. All this has of course to do with the fact that communication in the two media is subject to quite different conditions (II).

We have already pointed out that speech precedes writing fylogenetically and ontogenetically, and that the written code is largely secondary to the structure of the spoken language from a structural-linguistic point of view. But speech is not primary in all respects, and writing is not always merely a secondary reflection. Some features of the written language are clearly structurally prior to features of the spoken language. To use but one well-known example, written forms sometimes give rise to new speech forms, rather than vice versa; e.g. [ วu kê] for O.K., [ t trn $\left.^{h} \mathrm{ij}\right]$ for 10 p. ("10 pence"), [ phij eits dij] for Ph.D. ("doctor of philosophy"). In general, there seems to be a bidirectional interaction between the competence to speak and understand speech, and the competence to write and read. It is highly likely that the competence for spoken language of a literate person differs from that of the illiterate. Unfortunately, there are too few empirical studies of what happens to people's total linguistic competence when they gradually become more literate. However, there are some hints that our phonological intuition may change as a consequence of learning the alphabet (for many persons it is, for example, very difficult to imagine the pronunciation of a word without bringing its conventional spelling into conscious attention), and the actual pronunciation also changes at some points (cf. so-called spelling pronunciations). At the grammatical level, the acquisition of written standard language sometimes provides a person with a much more "elaborated code" to be used also in speech (cf. XI.2). Yet, such phenomena are seldom discussed in terms 
of competence for spoken language vs competence for written language. Instead, we have been accustomed to talking about one monolithic competence, that of the ideal speaker-listener (note: not the ideal writer-reader). ${ }^{6)}$

By way of conclusion, the study of linguistic performance is certainly not linguistically uninteresting and must be pursued if we want to understand the true nature of language. It is necessary to consider speech and writing as two different media, each with its own characteristics which are worth while investi-

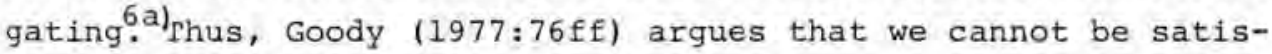
fied with only one distinction langue vs parole, or competence vs performance; he argues for at least three basic concepts, language (langue), speech (parole) and writing (écriture). One may add to this that it might also be necessary to split up the underlying language system into two distinct but largely overlapping systems, "la langue de la parole" and "la langue de l'êcriture".

Saussure and Chomsky are generally regarded as the most influential theoreticians in 20 th century linguistics. Saussure founded modern linguistics, and Chomsky revolutionized it.

No one can have avoided noting that Saussure's concepts of langue and parole has played a major role in our discussion so far. Similarly, some of Chomsky's concepts recur in our discussion. There will be several opportunities to return to their concepts later on, and we will see that the changes brought about by their "revolutions" in linguistics were not as sweeping as we often like to think.

6) Some modern psycholinguists work with several notions of competence. For example, Fodor, Bever \& Garrett (1974) make use of at least three constructs, i.e., the grammar (Chomskyan competence), the idealized sentence recognizer and producer, and the real (sic!) sentence recognizer and producer. However, the differences between speech and writing have nothing to do with this trichotomy.

6a) In very recent years, a few books have appeared which bear witness to the fact that this is a view which is currently gaining ground among students of language. See e.g. Baron (1981), Tannen $(1982 a, b)$. 


\section{V.3. Language as a set of products}

"When words and sentences are written down, they can be readily looked upon as objects"

(Lakoff \& Johnson 1980:204)

Speech consists of transient, dynamic behavior distributed and limited in time. The transience of the products of the activities of utterance production and comprehension make a processoriented approach seem natural. In other words, we should focus on the behavioral activities themselves. The interpretation of linguistic behavior is heavily dependent on an on-line interaction with background knowledge, non-verbal signals and various other features of the situational context. Written texts, on the other hand, consist of permanent object-like products which tend to be relatively autonomous, explicit, and subject to less variation than speech (cf. III). It is quite obvious that the linguists' conception of language structure and linguistic items squares rather well with the latter phenomena, whereas its adequacy for the analysis of spoken language and dynamic speech behavior is hardly beyond dispute.

The view that a language consists of a set of thing-like products is a recurrent theme in the linguistic literature. One of the most well-known definitions is the following:

'From now on I will consider a language to be a set
(finite or infinite) of sentences, each finite in
length and constructed out of a finite set of elements.'

(Chomsky 1957:13)

One of the most remarkable things in this statement ${ }^{7}$ is that a language is regarded as the set of all the linguistic products that can be constructed according to certain rules. An alternative, and intuitively more satisfactory, view would be that a

7) I will discuss other aspects of Chomsky's conception of a language later on, cf. esp. VI.1, VI.3, VII.8. 
language consists of all the units and rules which make up the system underlying the products. From the vantage point of the language user one could propose a more psychologically based definition; the language of a speaker/listener is his knowledge of the underlying language system (la langue), or better, his ability to produce and comprehend an infinite set of utterances, discourses and texts, which fit the underlying system of rules. This, by the way, would tie up with Humboldt's proposal that language be regarded as an activity (and an ability to act linguistically) (energeia) rather than as a set of products (ergon).

We will soon come back to some popular conceptions of the underlying language system and the speaker's linguistic competence. In the meantime I would like to return to the product type of definition. What is the source of the view underlying this? In Chomsky's case the inspiration came, at least partly, from mathematics, where a language is precisely a set of strings of symbols which are subject to a number of specific rules. (Note, by the way, that mathematical symbol languages are a highly specialized kind of written languages). But there are deeper and more traditional roots. Derwing $(1979: 165)$ wants to derive some of the characteristics of American linguistics, the emphasis on products (rather than processes and underlying capacities) and the view of language as something autonomous (cf. V.6), from Bloomfield's methodological recommendations. Bloomfield argued that linguists must concentrate on the structure of overt behavior, since we cannot speculate on the underlying processes out of lack of the necessary knowledge of physiology and psychology. However, while this may well have had an impact on American linguistics (including Chomskyan generative linguistics) ${ }^{8}$ ), it is surely not the whole truth. The fact is that linguistics has always been biased towards analyzing products, viz. written sentences and texts.

8) There are more similarities between Chomsky's and Katz' psycho-linguistics and variants of multi-stage behaviorism than is usually thought. See Linell (1979b) for some points. 
This deep-rooted tradition of course influenced Bloomfield and his contemporaries, although they were able to legitimize the product view on other grounds too (as Derwing points out).

\title{
V.4. Linguistic structures as closed hierarchical systems of things
}

\begin{abstract}
'Formalism and systematicity are the typical distinguishing marks of any kind of thinking focused on a readymade and, so to speak, arrested object'
\end{abstract}

(Volosinov $1973: 78$ )

\begin{abstract}
My next point is part of the very core of my argumentation. It concerns something which was outlined already in the Introduction ( $(1)$; linguistic structures are typically viewed as hierarchically organized systems of objects.
\end{abstract}

Lakoff and Johnson (1980:204) summarize "the premise of objectivist linguistics from its origins in antiquity to the present" as follows:

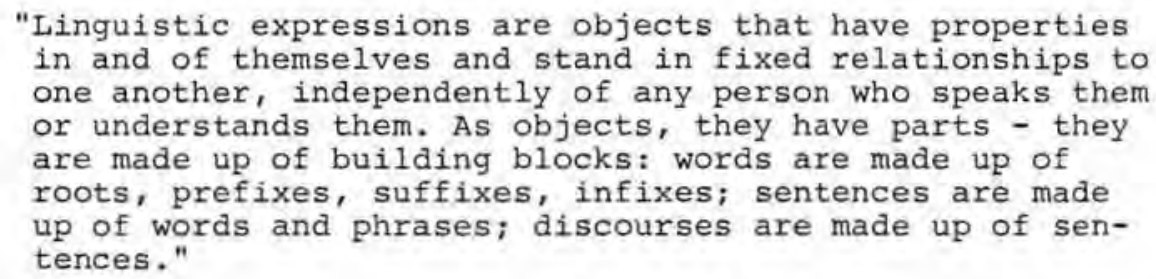

The "objectivist" view does not apply only to the linguistic products as such, i.e. what may be seen as the "linguistic data" (words, sentences, texts etc.); it is usually also expanded to cover "the underlying structures" underneath and beyond the "surface data", as well as to the linguistic code itself, i.e. the more or less permanent language system(s) consisting of items and rules and normally conceived of as "the grammar of the language". These last-mentioned constructs, i.e. la langue 
itself, will be discussed later on (see also V.5). Presently I will advert to the analysis of linguistic data (or the linguistic products) and their underlying structures.

The model which is most often applied to linguistic products, at least by (American) structuralists, is that of hierarchical constituent structure. Thus, the typical approach amounts to dividing linguistic products into successively smaller segments; the whole discourse (or text) is broken down into sentences, these in their turn into constituent sentences (main and subordinate sentences), clauses, and phrases, and phrases are thought to consist of words, words of morphs, morphs of syllables and/or phonological segments (vowels and consonants), and the latter are finally dissected into the "ultimate constituents", i.e. phonological features. A coherent text is thus pictured as a timeless web of part-wholes (proper constituencies) and other structural relations (dependencies of various types). The method of segmentation and classification has of course been applied mainly to intrasentential relations, but it has occasionally been applied to texts and discourses as well. In the analysis of face-to-face interaction, these methods have been termed "structural studies" (Duncan 1979) and considered to be typicalIy "linguistic". Duncan describes this approach to face-to-face interaction in the following way:

"A second approach to studying interaction structure is closer to traditional linguistic method. Data are generated through detailed transcriptions of face-to-face interactions. A wide range of actions is observed, and the beginnings and endings of these actions are carefully located with respect to each other. Strong regularities in action sequences are sought through systematic inspection (and sometimes statistical analysis) of the transcribed material. If the search is successful, the regularities in question are described in terms of hypothesized signals, rules, units, and the like.

It has been a characteristic of these studies to date that, while sequences of actions are the essence of the analysis, considerations of time per se are not included in the hypothesized structural elements. (Time may, however, be used in the task of transcribing the interaction, in order to locate interaction events.) The omission of 
time for the hypothesized interaction structure is not a principled aspect of these studies, but rather it reflects a general practice of current investigators. The practice may, and perhaps should, change as research continues."

(Duncan 1979:383-4)

It is worth noting that an outside reviewer like Duncan stresses the timeless nature of linguistic structures as particularly salient, and I shall revert to this point below (V.6).

If linguists analyze the observable patterns of linguistic data in terms of hierarchical constituent structures, this holds just as much for the underlying structures. Deep structures, semantic representations and morphophonemic forms are portrayed as hierarchical structures of thing-like (static, discrete, segments of various sizes. Furthermore, in Chomskyan mentalism these things are assumed to be "psychologicaliy real", and some of Chomsky's adherents, most clearly perhaps Katz (1964), have accordingly postulated a machinery of mental things that causalIy impinge on each other in the course of the "speech communication chain" ( $(I X: 1)$, Katz argues that the linguist "invents a theory about the structure of this mechanism, /i.e., the mechanism underlying linguistic communication/PL) ${ }^{\text {9) }}$ and the causal chain connecting the mechanism to observable events, to explain how these internal causes produce linguistic communication as their effect" (op.cit.:129). "The events to which the mentalist's constructions refer can stand as links in the causal chain that contains vocalizations and sound waves as other links" (ibid: 129-130). Thus, Katz clearly views speakers as more or less mechanical input-output systems; his "mentalism" is a paramechanism with a certain affinity to multi-stage $S-R$ behaviorism (cf. Linell 1979b).

9) Katz (1964) hypothesized that this "mechanism" included a generative transformational gramar, apparently in a rather straightforward fashion. 
However, it must be admitted that Katz' paramechanism represents a very radical view which has not been espoused by many linguists, not even among generativists. It is therefore a rather special conceptualization of linguistic objects. Returning instead to more common ground in linguistics, we may say that the view that linguistic structure are hierarchically organized object-like entities exists in its purest forms in structuralism. Saussure regarded language (la langue) as a closed integrated system of units (i,e, abstract objects or "things") with stable internal relations ("un système ou tout se tient"). The portrayal of language as an entirely rigid system, a structure within which items are arranged in fixed ways, was brought to an extreme in post-Bloomfieldian American structuralism. Later, generative linguists developed this radical structuralism along partly rather different lines. On the one hand, they preferred descriptions in terms of processes instead of merely items and arrangements, thereby doing justice to the recursiveness of the linguistic rule system. On the other hand, they expanded the universe of putatively existing linguistic objects; in addition to "surface units", i.e. actual word forms, sentences etc., they introduced "deep-structural" units into both syntax and phonology. Thus, abstract relationships such as morpheme identities between actual forms were thought to presuppose the existence of abstract morpheme-invariant phonological forms; i.e. in addition to word forms like English sane /sein/ and sanity/saeniti/ they (in this case: Chomsky and Halle 1968) posited a morpheme-invariant abstract form /s的/ $/^{10)}$. In syntax, surface structures and deep structures were thought of in analogous fashions. Thus, despite several obvious and important differences, it would be justified to conceive of Chomskyan linguistics as firmly anchored within the American structuralist tradition. (It is instructive to consult Hockett's (1968) characterizations of the "state of the art" in generative linguistics, e.g. pp. 31, 37, et passim).

10) Barring the general problems in treating linguistic elements as objects, this seems to amount to a category mistake (cf. Linell 1979a:ch 13). 
The theory that a language should be seen as nothing but a closed integrated system is a most unfortunate one, since it denies the significance of some of the most fundamental properties of language. Thus, adaptivity to new situations lies at the heart of language. Iinguistic variation is typical of linguistic communities, and this in turn is connected with the fact that all natural languages change over time. It is wellknown that extreme structuralism has great difficulties in accomodating such facts. According to these theories, "each historical change would have to be conceived of as a willful distortion of the inherited pattern, which is absurd" (Andersen 1969:828). Instead we must admit that languages, as well as speakers' competences in them, are open systems into which new elements can be introduced without becoming fully integrated. Therefore, a certain instability and certain conflicts may characterize them.

How is it that linguists look upon linguistic structures as hierarchically ordered, as it were spatially (two-dimensionally) arranged, static objects? It seems to me that the only really revealing explanation is to be found in the time-honored written language bias. Thus, I very much doubt that linguistic structuralism would have evolved in the way it has, if linguists had studied the varying, quasi-continuous speech behavior instead of the spatially arranged objects of written texts, and if they had not had access to the possibilities of organization and systematization inherent in writing (including listings, diagrams etc.) as a meta-language (cf. III:4). At any rate, the analysis of dynamic behavior might necessitate other conceptions of linguistic structure. Instead of thinking exclusively in terms of hierarchies of objects, other models and metaphors might be nevessary. Thus, if we look upon speech as a stream of vocalizations, as a stream of continuously progressing dynamic behavior, we might conceive of phonological and grammatical structure as sets of conditions superimposed on this carrier wave of vocalizations. Similarly, the function of semantic structure in thinking, perception, etc., may be understood in 
terms of conditions on streams of various kinds of cognitive activities.

One may also raise the question of whether the listener's anaIysis of behavior performed in actual language use is carried out entirely in digital terms. After all, that is the view that follows from most models in linguistics, cognitive psychology and artificial intelligence. The assumption of analogous processing seems fairly probable in some cases. Prosody, in particular the paralinguistic expressions of emotions (Fonagy 1977), is a case in point, but also many linguistically determined, phonetic elements may build upon dynamic wholes rather than sequentially ordered aggregates of static features. One may also generalize the argument and raise the question of whether perception in general involves global gestalts which cannot be reduced independent elements and structures (cf. Dreyfus 1972:151 ff.).

The common objection to the arguments that I just hinted at is of course the well-established contention that we always categorize and digitalize the objective reality in perception and cognition. Processing information via categorization has been said to be one of the most important organizing principles of human cognitive systems, and as such it would provide a natural basis also for structuralism in linguistics. While the force of this counter-argument must be admitted, it is still possible to argue on good grounds that structuralism is exaggerated in viewing language as a closed, integrated and surveyable structure in which each thing-like item has its carefully defined place. In order to substantiate this point a little more, I shall now return to the phenomena of linguistic variation and linguistic change for a moment.

\section{V.5 The invariance of linguistic structure}

Objects must necessarily have a considerable degree of permanence. Along with the view of linguistic items as thing-like phenomena goes therefore the view that their properties are 
fixed, stable, and invariant. It is assumed that under the bewildering variation in actual use there are some context-free and stable properties which are invariant over time and space. Hence it must be an important task for linguists to find out what these properties actually are (or should be).

This drive towards (searching for) invariance may appear in rather different disguises. If we look upon language as a set of norms for linguistic behavior, we may ask: What are the rules of proper behavior? What does the word $x$ actually mean? What is the correct pronunciation of the word $x$ (note: pronunciation in the singular)? In earlier times, the linguistic clergy (cf. III.2) often thought of their task as that of preserving the original, i.e. God-given, correct form and meaning of words. Today, the correct pronunciation and meaning is something that linguistic specialists determine by means of adequate analysis and properly constrained use of their own intuition. The normative perspective is therefore somewhat less salient; rather, linguistic items are treated as independently existing objects with objective (sic!) properties. However, the basic philosophy is still there; it is usually taken for granted that there are unique and stable phonological, gramatical and syntactic properties inherent in words and sentences, and, hence, that the search for these properties is a meaningful enterprise. It is assumed that under the rich dialectal, phonostylistic and idiolectal phonetic variation in the pronunciation of a given word, there is one unique phonological form. It is taken for granted that a given sentence has one well-defined syntactic structure, e.g. a specific constituency or dependency tree lor, in the case of syntactic ambiguity, a finite number of such structures). We like to think that the meaning of lexical items can be adequately described in terms of a finite set of semantic features or a hierarchical organization of such features, rather than, say, a more indeterminate and vague meaning potential (VII.2). Similarly, and perhaps even more questionably, sentences are assumed to have specific semantic representations defining "literal interpretations" of those sentences (VII.1, 3, 5). In 
mainstream contemporary linguistics, each sentence is assumed to be mappable onto a finite number of distinct meanings. That is, a sentence either does or does not have a particular meaning, a sentence either is or is not ambiguous, two sentences are or are not paraphrases of each other, etc. Yet, the study of the actual use of these sentences calls such an analysis into question; it appears that semantic interpretations are often more-or-less plausible. Rather than being unequivocally correct or absolutely excluded, many interpretations are more-or-less justified (cf. Sadock 1979).

The assumption of underlying invariance is clearly made also in modern psycholinguistics and cognitive psychology: language is regarded as a reified cognitive structure with fixed components. The kernels of linguistic items are constituted by the distinctive invariant properties, which are supposed to be clearly different from the redundant features. Thus, underlying phonological forms comprise a small and fixed set of phonological features, and word meanings are assumed to be simply set of essential features (necessary and sufficient conditions for some exemplar to fall under a certain concept, cf. VII.2). It is obvious that this approach obfuscates the facts that a given language is based on social and cultural norms and conventions, and that these norms are applicable in different degrees to the varying social contexts. Instead, linguistic rules are construed as mental processes (cf. VIII.3).

In spite of this, the currently popular conceptions of linguistic structure involve normativity, although in a much more subtle and less obvious way than the old view that linguistic rules were instigated by God. What happens today is that the linguists, rather than God, dub certain stable features the true, distinctive features and rules of semantics, grammar and phonology, thereby normatively disqualifying a great amount of variation as "linguistically insignificant" ("exclusively pertaining to performance", "linguistically irrelevant" etc.). 
Normativity, stability and invariance are features which are much more characteristic of the use of the written language than spoken communication. The linguists' attitudes to their objects of study can thus be at least partially explained by reference to the written language bias. In addition, it may be argued that this bias has been endorsed in recent years by the computer revolution in linguistics, especially as regards psycholinguistics and artificial intelligence. When we try to model linguistic structure and the use of language in reasoning on a computer, we have to use specific implementations and representations; computer programs must not be vague, and therefore, we choose a particular representation (or a finite number of such specific representations). It may then be tempting to assume that human beings are similarly determinate on all specific points. Such an assumption must be considered highly implausible (cf. VII.9). Moreover, disregarding this point for the moment, it is a significant fact that computers basically represent an expansion of the written, rather than the spoken mode of communication.

\section{V.6. The atemporality of linguistic structure}

As we have seen, linguistic items and structures are usually regarded as being static and invariant. Along with these properties goes another property, viz. that of atemporality. Iinguistic structures, such as semantic, syntactic and phonological representations, lack a time dimension; they are atemporal in nature (cf. V.4).

Now, linguistic structures are indeed abstract, and like other abstract phenomena, specifically various kinds of norms and rules, they are atemporal in an important sense. It would therefore be a category mistake to think of them as, e.g. behavioral processes. Instead, they may be construed as abstract conditions that are superimposed on the stream of behavior lor the stream of consciousness and/or other mental processes), However, in con- 
temporary linguistics the abstractness and atemporality of linguistic structure are conceived of in quite another manner, viz. as mental objects which exist simultaneously in their entirety; exactly as real physical objects, including written texts, they are (in principle) invariant over time. Thus, despite the fact that speech consists in a dynamic behavior distributed over time, linguists (and psychologists) look upon phonological, syntactic and semantic "representations" of individual utterances just as simultaneously existing mental objects.

Most psycholinguists are careful to distinguish between the linguistic structures as such (which are atemporal and simultaneously existing) and the processing involved in the linguistic activities of utterance production and perception. The processing is of course distributed over time. What is important, however, is that (psycho-)linguists tend to conceive of this processing as consisting in the construction, analysis, and manipulation of (simultaneously existing) mental objects, i.e. linguistic forms and structures. This is particularly true of the kind of mentalism that inheres in orthodox generative psycholinguistics. The time-distributed processes involved in utterance production and perception are assumed to involve the accumulation and integration of structures, e.g. syntactic surface structures, in a short-term memory, where they are supposed to be simultaneously accessible for inspection and analysis in their entirety. Many of the common testings in psycholinguistics are characterized by these assumptions: ${ }^{11}$

"A direct consequence of assuming a transformational model of linguistic representations has been a general lack of interest in sharpening up our picture of the temporal properties of sentence processing. ... For if the point

11) Cf. also Goldman-Eisler $(1968: 2)$ who argues that a tendency to spatialize and detemporalize events appeared when psychology was developed in the direction of experimentation and quantification. In accordance with the main lines of argument of this book I would say that the roots of this "detemporalization" goes much further back in the history of psychology. 
of contact between the input and the internal representation of linguistic knowledge is only properly dealt with by the linguistic theory when entire clauses and sentences have become available, then it is only natural to focus one's research attention on what happens at these points, and to use experimental techniques appropriate to this interest. Namely, post-sentence measures, such as sentence grammaticality judgements, sentence verification tasks, sentence anagram tasks, memory-probes, and the like, which reflect the subjects' internal representation of the input sentence as a whole, but which can only be weakly sensitive to whatever happens while the subject is listening to the sentence in the first place. The results of experiments using post-sentence measures do not give us the kind of information about on-line processing that an adequate processing theory requires."

(Marslen-Wilson 1976:206)

These remarks of Marslen-Wilson's seem quite to the point. Notice that this does not amount to a denial that assembling of information in short-time memory does occur in speech processing. The point is simply that the prevailing theory (which, I think, is ultimately dependent on the written language bias) may prevent the psycholinguist from seriously trying out alternative models like those involving interactive on-line processing. See also $\S I X . l$.

\section{V.7. The autonomy of language and linguistic structure}

I have already commented upon the common view that language is a stable, autonomous system which can and should be seen as immanent, sufficient in itself, and independent of other factors, e.g. the contexts where language is used. We also noted the corresponding methodological stand according to which linguistics should be pursued as an abstract discipline analyzing the relations between different linguistic items and products without regard to contextual modifications and the interplay with other conditions of use (so-called autonomous linguistics). I have also pointed out that these conceptions are more naturally at hand for a linguistics based on written discourse than for a study of communication by means of speech. I would now only add 
a few remarks concerning the consequences of this type of linguistics.

Autonomous linguistics implies, first of all, that language structure can be studied without regard to the use of language. According to Saussure and Chomsky, the study of la langue and the ideal speaker's competence are logically prior to any study of parole and performance. This has led to the Chomskyan division of labor between linguists and psychologists: first linguists determine what is assumed to be the speaker's linguistic competence (and this is done by autonomous linguistic-structural methods), and only then are psychologists (and psycholinguists) encouraged to investigate how this 'competence' is put to use in performance. It is nowadays widely recognized that this procedure is impracticable, and that structure and use, competence and performance must be studied in parallel and not in isolation from each other.

A related consequence of the structuralistic ideal of immanence is the hope that language use and linguistic competence can be isolated from other mental activities and capacities. But if we approach the complexity of speech communication with open eyes, prepared to observe the intricate interplay between verbal utterances and the surrounding context of background knowledge, non-verbal signals, and reliance on physical and social-psychological features of the situation, we realize that this is a vain hope. On the other hand, it may seem less absurd as long as we as linguists concentrate on written texts, which are more independent of specific senders, receivers and contexts.

For the study of linguistic communication, the linguists' isolationism has had many important consequences, some of which have - in my view - been rather unfortunate. For example it has enhanced a view according to which the structures of language and speech are radically different from all forms of non-verbal, e.g. paralinguistic, communication, and this has precluded the possibilities of searching for common sources and explanations 
valid for both verbal and non-verbal communication. Compare, e.g. Chomsky's (1968:70ff.) denial that there are any symbolic systems comparable to language within the domain of non-verbal communication. In particular one should deplore the consequences that this view has had for the theories of the child's language acquisition. Jakobson (1968) argued that there were no interesting relations between babbling and phonological development; we know from developmental psychology that there are successive developments from non-verbal gestures to verbal ones (Werner and Kaplan 1963). Chomsky's belief in the absolute uniqueness of natural language led him to postulate a highly specific, innate faculty of language, when in fact many features of language can be at least partially explained by relating them to various forms of non-verbal communication.

\section{V.8. Norms and normativity in linguistics}

When linguists were involved more or less directly and exclusiveIy with teaching people a correct language or with developing new written standards of so far insufficiently standardized national languages etc., their discipline was of course rather explicitly normative; it simply prescribed rules to be followed in the use of language. We can also put the point in quite general terms; a normative focus is natural for a linguistics dealing with written language. Learning to write makes a great deal of education necessary, and this consists in no small measure in the learning of conscious norms. On the other hand, a person's spoken vernacular is acquired without any explicit instruction, and it may be argued that it is in fact constrained by fewer grammatical rules than written language. Yet, as we will see, the norms of written language enter the analysis of spoken discourse too.

In today's linguistics analyses are carried out more for purely theoretical reasons. It is generally held that the aims of linguists are nowadays plainly descriptive (and, possibly, explanatory); rather than prescribe how people should act 
linguists (and other social scientists) are assumed to describe how they do in fact act. Nevertheless, our linguistic practice is still replete with normative aspects, although this is rather seldom admitted ${ }^{121}$. One should therefore talk about the hidden normativity of descriptive linguistics.

The hidden normativity shows up at different levels. For example, anyone who really pays attention to the full variability that actually characterizes spoken language, may well be amazed by the fact that many of the actually occurring structures have not been described in the usual grammars, not even in modern grammars compiled by "descriptive" linguists. The most important reason for this is probably the fact that the structures in question are not accepted in written language. Hence the most natural reaction is to regard such structures as ungrammatical or deficient. (The history of language cultivation is full of examples.) But once some such structure is attended to and described by some linguist as part of spoken language, it is thereby assigned another status; it becomes recognized as part of the language in question and thereby, as it were, legitimized. This phenomenon - quite frequent in modern linguistics - clearly shows the normative function of the descriptivist practice.

But normativity cuts both ways in current descriptive linguistics. Perhaps the normative aspects are most perspicuous in other ways, e.g. in our assessments of various constructions of spoken language, as, for example, when so-called anacolutha are discarded as ungrammatical, i.e., as not belonging to the

12) This is particularly true of conventional introductions to modern linguistics. These very often contain a short introductory chapter on the history of linguistics, in which it is simply asserted that linguistics used to be prescriptive whereas modern linguistics is descriptive, Yet, such textbooks are heavily prescriptive in that they tell beginners how they should understand language and how linguistics should be done (according to the particular author's theoretical preferences). 
language involved (language defined according to Chomsky, cf. $\mathrm{v} .3)$ although they are perfectly natural and function well in their own medium. The norms which are explicitly or implicitly applied by linguists in such enterprises are quite often such norms as have been consciously adopted by normative grammarians of the past in their regimentation of written standards (VI.3). There is always a temptation to regard as ungrammatical such stretches of spoken languages which cannot be subsumed under the generalizations which follow from these rules (or norms).

Furthermore, linguistic analysis is of course subject to norms that belong to the metalangauge used. Another way to put it would be to say that analyses are dependent on the very special language games which are performed by the linguist as he applies his methods to the data. The metatheory to which a given linguist adheres allows only of certain kinds of "linguistic representations", e.g. certain types of syntactic constituency trees or dependency structures, or certain kinds of underlying phonological representations (e.g. linear configuration of segments, cf. VIII.1). Thus, such meta-theoretical norms will part$1 y$ determine the linguist's identification of invariant properties behind the variation in linguistic data.

By way of summary, linguistics is still normative in nature. There is nothing surprising or indecent in that; all sciences are to some extent dependent on norms, and in the social sciences the situation is particularly complex, with norms permeating both data and (meta) theory. 
VI. GRAMMAR

VI.1 The concept of sentence

I have already pointed out that grammar is a sine qua non for classical as well as modern linguistics. I will now discuss some salient features of modern grammar theory. A convenient point would then be the Chomskyan definition of a language as a definite (infinite but enumerable) set of grammatical sentences (cf v.3). There are at least three debatable features of this definition. The first is the view that a language is considered to be a set of products (written or spoken sentences) rather than underlying activities, conditions on such activities, resources utilized in such activities, or the like. This has been discussed in $\S \mathrm{V} .3$. The second point, the issue of grammaticality, will be treated in $\S$ VI.3. Presently I will turn to the third aspect, the status of sentences. ${ }^{1)}$

Historically, there are two categories of linguistic units that have been considered much more important than others, i.e. words and sentences. Why is it that Chomsky and many others, indeed the majority of modern linguists, take the sentence to be the self-evident basic unit? This question can hardly be answered unless we attempt an analysis of the concept itself: How should 'sentence' be defined?

It is quite obvious that rather different conceptions of what a sentence is have dominated different epoques in the history of linguistics. Nowadays, the sentence is usually taken to be

1) Note also the characterization of a natural language as if it were a formal language (VII.8). 
a form-based concept, but traditional characterizations are often more vaque and content-based. I will first consider some of these conceptions.

A time-honored definition amounts to regarding the sentence as 'the expression of a complete thought' or the like. Is this a workable definition? For example, does it make sense to say that a discourse comprises a sequence of (expressions of) complete thoughts? Is a language really an infinite but enumerable set of such expressions? The answers must be no, and the reasons are quite well-known from the linguistic literature. I will therefore be content with noting two difficulties. One has to do with the fact that it is hard or even impossible to tell what is meant by saying that a thought is "complete". Instead a more reasonable thing would be to say that any given 'thought', whether overtly expressed or not, can always be further specified, made deeper or more precise. No linguistic expression is ever semantically autonomous or complete in itself; in order to be interpreted and understood it always needs a certain amount of pragmatic specification (more about this in § VII.1). Accordingly, we can hardly rely on notions like '(expressions of) complete thoughts'.

Another objection to the classical definition of the sentence is that it seems counter-intuitive to argue that linguistic expressions, or utterances, always express 'thoughts' (unless one is willing to subsume under this term everything indicative of some mental act on the part of the speaker):

'As applied to many samples of speech, the description is... grotesque. Consider a mother anxiously asking for news of her son, or a tradesman driving a hard bargain. Or again, imagine an angry traveller hurling words of abuse at an uncomprehending porter, or a judge pronouncing sentence of death upon a murderer? Shall we say that these persons are expressing thoughts?'

(Gardiner 1951:17) 
It seems inaccurate to argue that utterances such as Fire!, How do you do, Mr. Swanson and I christen Thee Andrea Doria are expressions of thoughts, whether complete or incomplete. Moreover, the theory of sentences as expressions of thoughts is embedded in a general theory of utterance, which says, in essence, that the speaker first forms a thought, and then verbalizes it as an overt utterance. This theory is unrealistic for several reasons (§ IX.1).

Accordingly, Gardiner and many others opt for a more general and still very vague definition of sentence. In fact Gardiner uses the term more or less synonymously with 'utterance'. He proposes (op.cit.:98, 182) that a sentence is an utterance which is "completed" "to the satisfaction of the listener", i.e., the listener feels that he has been told something when he has heard and understood a 'sentence'. Again, we are faced with an unduly vague notion of sentence. Therefore, one is tempted to opt for a more formed-based concept of sentence. This is all the more motivated, because we will need several concepts, including a distinction between 'utterance' and (something like) 'sentence' 2) (more about this need below).

The concept of 'utterance' is not easy to define, and I cannot give but a very loose characterization here. Thus, I shall mean by 'utterance' a stretch of speech (by one speaker) which is formally relatively independent; it is grammatically and prosodically marked off from the context and does not contain any major internal prosodic or grammatical breaks. From a grammatical point-of-view an utterance may have various structures (phrase, clause, sentence etc). Some scholars prefer other terms instead of 'utterance', e.g. 'Iocution' (Kendon 1980),

2) In fact, Gardiner himself confuses these two concepts, as he concedes himself (1951:336-7) 
'macro-syntagm' (Loman \& Jörgensen 1971), 'macro-segment' (Hockett 1958) etc. ${ }^{2 a)}$

However, we are still not finished with the content-based definitions of 'sentence'. There are still more variants, e.g. those which are based on notions like 'proposition' or 'subject-predicate structure'. It seems reasonable to argue that every message, however simple, must logically contain at least a logical (or psychological) subject and a logical (psychological) predicate, i.e. a 'theme' and a 'rheme', a function with at least one argument, a proposition ${ }^{3)}$, or else it would simply not be a message.

The primacy of propositions is endorsed by many linguists, logicians, and psychologists. It is part of Wund's theory of "the psychology of the sentence", and one of the great pioneers of aphasiology, Hughlings Jackson, declared that "the unit of speech is the proposition - to speak is to propositionize" (1878). Perhaps, this may be interpreted as evidence for the sentence as a unit of discourse? Again, the answer has to be in the negative, and this briefly for the following two reasons. First, if the proposition is the semantic unit, then the corresponding expression would be the clause for simplex (main) sentence), i.e. a simple association of a logical subject and a logical predicate, rather than the sentence (as the term is normally understood, cf. below). Furthermore, it must be emphasized that the proposition is a semantic or communicative unit rather than a unit of expression. Its parts need not be overtly expressed. In the following fragment of a dialogue, B's utterance may be said to embody or presuppose a proposition (roughly 'I live in London'):

2a) The term 'utterance' is often used in another sense, viz. ' the entire stretch of speech produced by a speaker in one single turn of speaking'. This sense of the term will not be discussed here.

3) I am, of course, aware that these concepts are not equivalent, but the differences seem immaterial to my present argument. 
(1) A: Where do you live?

$B$ : In London

In other words, the expression In London must be further specified (using the overall context) in order for the listener to "reconstruct" a proposition. However, such pragmatic specification is always necessary; it applies to (1B) and (2B) alike, although we would say that only the latter is a sentence (and then we presuppose, of course, a form-based notion of sentence):

(2) A: Do you live in London?

B: $($ Yes, I do.

It is implicit in the preceding discussion that 'sentence' should be defined by reference to formal-structural properties, and that such a concept, distinct from that of 'utterance', is in fact needed. Briefly, sentences are a category of linguistic expressions with the following characteristics:

a) a sentence must be limited by an onset and an end that are structurally clearly manifested, 4)

b) a sentence must, at least in some languages, e.g. English, contain both a subject and a predicate 5 )

c) a sentence must exhibit certain other properties which enable it to occur as an independent unit. Compare the difference between a matrix sentence (main sentence, Hauptsatz) which is a true sentence, and a subordinate clause (or subordinate sentence, Nebensatz) which is not a true sentence (but a clause).

d) a single sentence may contain several clauses, e.g. subordinate clauses.

4) One might say that there has to be some type of counterparts of the initial majuscle and full stop (point) of the written language.

5) I abstain from discussing whether or not imperatives are complete sentences. 
Since utterances are basically stretches of speech, whereas sentences (in the normal modern sense adopted here) are abstract grammatical units (configurations of grammatical conditions), the two units are actually incommensurable:

'As a grammatical unit, the sentence is an abstract entity in terms of which the linguist accounts for the distributional relations holding within utterances. In this sense of the term, utterances never consist of sentences, but of one or more segments of speech (or written text) which can be put into correspondence with the sentence generated by the grammar.'

(Lyons 1968:176)

Therefore, with this type of definition, sentences are strict1y speaking not performance units. However, we could try to analyze language use at the grammatical level and then find out whether a given text or discourse can be characterized in terms of sequences of spoken or written words (text sentences, "sentence tokens") fulfilling the conditions of sentencehood at the abstract grammatical level. Actually, one should keep 'sentences' qua abstract gramatical entities and 'text sentences' terminologically apart, but for convenience we often refer to text sentences (sentence tokens) simply as 'sentences'. Thus, a text or discourse can be analyzed in (text) sentences. if there are clearly identifiable boundaries between the units in question, and if these units display a clear internal structure, including clear differences between main clauses and subordinate clauses. It turns out that these conditions are often simply not fulfilled in normal spoken discourse. In spoken communication we often find a sequence of clauses and phrases which are rather loosely strung together without there being any clear boundaries or differences between clauses of different kinds. In other words, the clause is undoubtedly one of the units of grammatical analysis applicable to casual speech, but the sentence is only characteristic of certain discourses, especially those which are heavily influenced by the speaker's knowledge of elaborated written language.

This means that the sentence is a unit of analysis which is best suited for written discourse. An essential part of the 
laborious task of learning to write properly consists precisely in the requirement to become capable of organizing one's inguistic output in complete and well organized sentences. In casual speech such structures are not natural. Yet, the linguists analyses of spoken discourse are typically constrained by the search for such structures even where they cannot be found. This approach has several unfortunate consequences. For one thing, many structures are condemned as being ungrammatical, faulty, illogical etc, simply (it seems) because they do not meet the requirements for sentencehood. Secondly, many nonsentences are treated as sentence fragments, and the gramuar is typically made to derive them from underlying sentences. After all, the only initial symbol of an orthodox generative grammar is the $S$. Thus, for example, In London of (1B) is obligatorily derived from a deep structure corresponding to 'I live in London'. This type of analysis is in many ways unfruitful:

'Conversation is a text type replete with surface structures which are not in fact sentences (McCalla:14). Consequently, the study of concersation in the framework of sentence grammars both (a) misses the real issues (actions and goals) and (b) creates difficult if not unresolvable non-issues (how to convert everything into well-formed sentences)' .

(Beaugrande 1979:1)

Therefcre, if we attempt something like a generative grammar of spoken discourse ${ }^{6)}$, it should (base-) generate many different kinds of phrases and clauses that are not sentences. Sentences in the technical sense are only seldom encountered in many natural forms of talk. It seems that the ideal of using only or mainly sentences is something which has gradually evolved with the development of written standard lanquages. It may be interesting to observe that sentences (in our sense) were not as

6) The idea of such a generative text gramar is actualiy, if taken seriously, a deac end (cf.e.g. VI: z) 
obligatory in the written language of the past. For example, Plato's dialogues contain a lot of anacolutha, which would now be rejected as ungramatical, and the same applies to Shakespeare's plays. Early Middle High German texts were replete with structures, which were later relegated from written Standard German. However, the same types of constructions have existed all the time in spoken German (Sandig 1973).

There have been strong elements of regularization in the regimentation of language which has been performed by language cultivators (i.e. prescriptive linguists) when they have tried to develop written standards on the basis of spoken vernaculars (and various classical languages). In general, this has involved a predilection for complete, symmetrical, non-elliptic sentences (or clauses) with a clear grammatical structure (one subject, one predicate verb, a full verb complementation, a certain basic word order etc) and clear differences between matrix sentences and subordinate sentences (clauses). For example, in the grammar tradition for German and Swedish one can discern an endeavor to define various clear, gramatical differences between matrix and subordinate clauses, although these differences have never been very clear-cut in natural spoken discourse. ${ }^{7)}$ Today, similar trends towards regularization of data appear in the linguists theoretical analyses, e.g. when non-sentences are derived from underlying sentences (cf above).

The preoccupation with sentences in contemporary 1inguistics has made it practitioners unable to cistinguish properly between sentences, which are, or should be, defined in terms of certain formal traits, and utterances, which may take on various

7) On matrix vs subordinate sentences in German and Swedish, see e.g. Sandig 1973 and Andersson 1975, respectively. 
kinas of overt forms. It is commonplace in contemporary psycholinguistics, particularly in the U.S., that the use of the language, speech performance, is talked about in terms of the production and comprehension of sentences, not of utterances (e.g. Fodor et al. 1974, Rosenberg 1977, Eoss \& Hakes 1978). One may argue that this is a rather innocent example of sloppy terminology, but it seems to me that it invites the idea that sentences (in the strict, form-based sense) are indeed produced and understood in spoken discourse. At least it is implied that the underlying structures are sentences, since the occurring non-sentences are treated as sentence fragments and grammatically analyzed as derived from underlying sentences.

If some linguists still believe that spoken aiscourse consists of sentences, this is of course even more true of laymen. After all, one learns at school that only complete sentences count as correct language.

Even if it is a wide-spread view that spoken vernaculars are wrong (of. XI.2), it is still generally believed that it would be relatively easy to identify the spoken counterparts of such things as the sentences and punctuation marks of the written code. Hence, when this is not borne out, the tendency is to assume that the language being analyzed is deviant from normal language. For example, such a view seems to be inherent in Maher's (1972) analysis of the language of schizophrenia. 


\section{VI.2 The neglect of prosody}

There is hardly any doubt that prosodic phenomena like stress, tone, intonation and durational phenomena are very important cues in speech, for example as regards the foregrounding vs backgrounding of various elements of meaning. In addition, emotions and attitudes are expressed by the use of related, socalled paralinguistic phenomena, i.e., variations in melody, tempo, pausing, loudness, register, articulatory accuracy etc. However, most of this has no counterpart at all in written texts. Note, incidentally, that the communicative functions of prosody (in the wide sense) are emotive, evocative and social rather than cognitive and referential, and only the latter, more "intellectual" functions have traditionally been regarded as typical of human language. Again, this is in full agreement with the written language bias (III:1).

Accordingly, one is tempted to say, linguists have been very little interested in prosody, and our current theories of prosody are clearly insufficient. It is true that prosody seems to be extraordinarily hard to come by, and the analysis could hardly be performed without modern instrumental equipment like tape recorders (sound and video taping) and computers, but there is hardly any doubt that the written language perspective is also partly responsible for the neglect of prosody.

The effects of the neglect of prosody can be seen in several subfields of linguistics. It is evident in gramar theory, where our data are almost always only written sentences (considered out of context), although this restriction is selcom pointed out. As an example, consider the numerous cases of ambiguous sentences, the analysis of which has played such a crucial role in the argumentation for deep structures and transformations in syntax. In many of these cases, the different readings (note the word 'reading'!) have, or at least can have, different prosodic patterns, and therefore we presumably have 
distinct surface structures in speech. Here are some well-known examples:

(3) A: Flying planes can be dangerous

B: He has plans to leave

C: What disturbed John was being disregarded by everyone.

D: The police were ordered to stop drinking after midnight.

The orthodox generative literature maintains that these sentences have several readings, and these readings with their distinct meanings are disambiguated in speech. Note the written language perspective underlying this reasoning; the linguistic objects, i.e. the sentences are treated as being ambigous as such, but distinct readings may be signalled in speech. But if speech is primary ( $f(\mathrm{~V}: 2)$, it would seem natural to talk about ambiguation in writing, rather than disambiguation in speech.

What is perhaps even more remarkable is the fact that the neglect of prosody characterizes psycholinguistics just as much. In research on the perception and comprehension of linguistic messages, one normally uses written sentences (in isolation), as if the perception and comprehension processes involved in reading were only trivially different from those of listening to natural speech. The same is true of research on memory effects, in artificial intelligence etc; prosody and its possible effects are almost never taken into consideration.

In his discussion of the merits and drawbacks of some current models of perceptual strategies, Welin (1979:45) notes:

'A fourth problem is the implicit orientation of these models towards the perception of written text, although they

8) Welin here alludes to the work of Frazier \& Fodor 1978, but his points are generally valid for most of the standard psycholinguistic research on the perception and comprehension of linguistic texts. 
are claimed to be valid as models of the listener as well. The use of intonational patterns and pauses in natural speech is not discussed, and it is generally assumed that the input is already segmented into 'words'.

Yet it is of course far from true that the isolation of words can be performed automatically and in the same way as graphic words are separated in writing (cf VI:4). Again we see that the only problems which are seriously considered by linguists tend to be those which are evident from the study of written communication. 


\section{VI,3 The concept of grammaticality}

The functions of linguistics in past times provide a natural explanation for the fact that grammar has always been the focus of linguistic theory (V.I) The practical goals of traditional linguistics also made a concentration on the norms of grammaticality seem motivated and indeed necessary; it was important to propose more or less explicit norms for how a standard language should be written.

In present-day theoretical linguistics, ospecially in the Chomskyan tradition, the rules for grammaticality have a somewhat different but in no way less important function. If we assume a language to be a definite set of linguistic products, i.e, a set that can be exactly characterized (generated) by a finite set of formal rules (V.3), then these rules (or norms) of grammaticality will be of the utmost theoretical importance. In fact, generative linguistics is in a way more normative than most of traditional linguistics. Whereas most traditionalists probably regarded the living language as a rather diversified array of linguistic products, in which the boundaries between grammaticality and ungrammaticality were at least partly quite fuzzy and not amenable to any exact characterization - after all, this was one of the reasons for establishing explicit norms for the written standard language -, generative linguistics assumes that it is indeed always and everywhere possibie to determine the exact limits of grammaticality on empirical grounds, at least if one is prepared to allow for a certain amount of idealization.

There is today a growing awareness also among theoretical inguists that the traditional assumption of fuzzy boundaries was after all well motivated, and that the idealization involved in establishing the Chomskyan construct of competence with its exact rules is intolerable because it distorts the empirical facts beyond recognizability. Of course, no one would deny that there are a considerable number of rules of grammaticality un- 
derlying spoken language. These rules clearly distinguish between grammatical and ungramatical strings in countless simple cases:

(4) A: He saw her

B: He did not see her

(5) A: Her saw he

B: Her see he not did

The sentences of (4) are undoubtedly correct English, whereas the strings of (5) can just as easily be dismissed as incorrect. This of course holds for spoken language too, and most native speakers of English, even young chilcren, would no doubt react negatively and correct the use of them (by e.g. foreigners). Thus, we should be able to characterize a number of conditions on correct English which would distinguish clearly gramatical strings (such as (4)) from clearly ungramatical strings (like (5)). However, the Chomskyan idealization is much more farreaching; it demands that it be possible to define rules of grammaticality for any types of strings, no matter how long and structurally complex they are. This follows from the Chomskyan definition of a language (V.3). Furthermore, since the clear cases are never decisive in the testings of various proposed systems of sophisticated generative rules, it is precisely the more complex strings which have come to play the crucial role in the grammarians' efforts to develop a complete generative grammar for a given language. There are innumerable examples in the generative 1 terature of very complex strings, the grammaticality of which is very difficult to decide. Here are some items: 9)

9) Stars and question marks are Ross's own. They seem to be used about both ungrammaticality and unacceptability. Although Ross subscribes in theory (1967:59, fn.8) to Chomsky's (1965) definition of grammaticality vs acceptability, he works - in practice - primarily with unacceptability. (As we will point out below, the distinction is indeed hard to maintain).

- Ross (op.cit.) characterizes (6) as relatively acceptable (of "reduced unacceptability"), (8) as "ungramatical", and (9) as "grammatical but of low acceptability". (7) is said to be a "tortured" and "less than felicitous". 
(6) ? I watched the Indians who the man who had been my advisor in my ireshman year had advised me to study when I got to Utah talk, because I was fascinated by the way their view of the world seemed to be constrained by the structure of their language.

(Ross 1967:39)

(7) 26 The contract which I want to peruse after copying by treating in milk while pressing between two pieces of marble in flattening out is a beautiful piece of art. (ibid:106)

(8) * Reports of which the government prescribes the height of the lettering on the covers are invariably boring. (ibid:111)

(9) ?? Which packages is it possible that Sam didn't pick up until it had stopped raining which are to be mailed tomorrow? (ibid:159)

(10) Which packages is it possible that Sam didn't pick up until it had stopped raining which he arranged with his agents in Calcutta to send to him here in Poplar Bluff because of his fear that someone in Saint Louis might recognize him? (ibid:159)

(11) Var det Södersjukhuset; som Hälsovărdsmyndigheterna lät undersöka vilka färskvaror; det var oklart vilken grossist somk $_{k}$ hade levererat-jill -?

'Was it the South Hospitali that the Health Department investigated which fresh producej it was unclear which firm $_{k}$ that $_{k}$ had delivered-jo $-i$ ?'

(Engdahi 1979)

It may seem very difficult to comprehend and accept complex sentences like these. Their gramatical stazus is hard, if not impossible, to determine on anything like "objective" grounds. Even the judgements made by professional linguists seem to depend on rather accidental events. Sometimes, the linguist succeeds in finding a context in which the sentence under consideration makes sense (and, hence, could possibly be used), and then it seems tempting to dub it grammatical. In other cases, the linguist has not (yet) succeeded in finding a suitable context, in which case the sentence will normally be 
starred as being ungrammatical. 10 )

For such reasons (cf. also below), controversies over the gramaticality or ungramaticality of very complex sentences have often turned out to be fruitless. Indeed, they seem to be unsolvable without recourse to rather arbitrary principles. One such way out is the assumption of different idiolectal grammars; when linguists cannot agree on the grammaticality of some given sentences, they may simply assume that they have "internalized" different grammars (different norms), which accounts for their differing judgements (cf. what Botha, 1973:219-9, calls the ' "my dialect"-"your dialect" gambit'). Another rather dubious principle is part of Chomsky's early proposal of "letting the grammar decide" ('Clear Cases Principle', cf. Chomsky 1957:13-13, Fillmore 1972). These attempts have met with a lot of criticism. A reasonable conclusion is that the whole issue of grammaticality, when applied to complex cases and in general to spoken language, is a pseudo-problem. It is futile to search for the precise set of grammatical rules underlying spoken discourse, simply because there is in many cases no such set of rules.

10) Ross (1967) declarec e.g. $(7,8)$ ungrammatical, while Engdahl (1979) found (11) to be grammatical in Swedish. Engdahl did so after providing a context where (11) could possibly occur, while Ross dic not discuss contexts explicitly.

However, in all fairness it must be pointed out that, in general, Swedish does seem to be more liberal in allowing so-called extractions out of relative clauses, i.e. in the type of examples considered here (Engdahl \& Ejerhed 1982). Such differences between languages are obviously not uncommon. But this does not change the main points of our arguments in this paragraph. 
Grammaticality is a rather peripheral property of structurally complex utterances in natural spoken discourse. There are of course clear rules for how simple words, phrases and sentences may be constructed, but when speakers are forced - due to complex contents or other situational factors - to use long and complicated linguistic expressions, these constructions are combined in various ways, and the results become more or less clumsy, more or less comprehensible, they may be more or less good vehicles for getting the messages across. Many such expressions are hard to assess for grammaticality; instead, one must concentrate one's efforts on finding plausible interpretations. When linguists try to use their intuitions for determining grammaticality, they very often fail. As a result, many utterances are discarded as impossible or ungramatical, unless - by some coincidence - someone comes up with a situational context where the utterances may in fact occur, in which case the juagements are often changed. In other cases, the linguists are forced to rely on more or less arbitrary rules of thumb lcf. above).

One of the reasons why the assessment of grammaticality becomes such an insecure endeavor is the fact that many spoken utterances look very clumsy, unnatural and difficult to interpret when they are given in written form. The context, which made their production possible, is gone, and the same holds for the prosody. Both may be quite difficult to hit upon in the absence of direct clues. Furthermore, there are fewer and less clear restrictions on word order in speech than in standard written language. Once a natural prosody is found many seemingly odd word orders turn into something quite natural.

It might be added here that the data which are discussed by linguists are most often made-up sentences and not utterances taken from authentic spoken discourse. This means that the examples are quite often edited in some respects; for example, the complex structures considered by e.g. Ross (1967) are typi- 
cally coherent and complete sentences, not such often incoherent structures (like anacolutha) as occur in actual spoken language. Here we see how the linguist's norms may even distort the very data, i.e. that which is to be used as empirical evidence. We witness the practice of regularizing data (VI.1)

The boundaries of grammaticality are fuzzy at many points. As I noted earlier, the main explanation for this is probably the fact that grammaticality is often rather unimportant at least in the life of spoken language. After all, normal dialogues do not consist in an exchange of arbitrarily chosen "grammatical sentences". On the contrary, utterances must, first and foremost, be situationally adequate and relevant at the particular stage of discourse where they occur. This raises the question of whether sentences can be considered well-formed in themselves, i.e., as independent formal objects (as is usually assumed if language is defined as a set of sentences), or if they should be considered "well-formed", "acceptable", "correct", adequate" or "plausible" (there are many such predicates that could be used) only relative to (classes of) meanings, interpretations and contexts.

Let us therefore briefly discuss the relative importance of the three conditions of situational relevance (which is basically a matter of semantics in the wide sense), coherence (and/or cohesion) 11) (which has to do with both semantics and syntax) and grammaticality (gramatical accuracy) (which is primarily a question of outer form). The first-mentioned condition spe-

11) Many scholars make a distinction between, on the one hand, coherence (coherency) meaning conceptual (semantic) connectedness and, on the other hand, cohesion having to do with formal markers of connectedness (anaphora, ellipsis etc). 
cifies that utterances mus $\tau$ be adequate and relevant in the particular aiscourse contexts in which they are embedied. The second concition means that utterances and discourses must be sufficiently coherent as regaras both content and form. Thirdly, utterances must in general exhibit certain features typical of what is conventionally regarded as correct in the language in question. In adation, speech must often also exhibit a considerable amount of fluency on the part of the speaker ${ }^{12}$ ). Without this he would run the risk of the listener's taking over the floor ${ }^{13}$ ).

Since communicative purposes and goals vary a great deal, the inherent importance of these conditions also varies across situations. Thus, for example, narratives, which tend to be relatively selfcontextualizing, have to rely very much on coherence, whereas informal interviews and small talk are often seemingly quite incoherent. Conventional constraints on the outer form are socially important in some cases, e.g., in solemn speeches and other rituals. In spite of this, however, it seems that situational relevance, coherence and gramaticality should be rated in this order with regard to their overall communicative import. Thus, in most situations situational relevance is much more important than grammatical correctness. Without that the speaker will not be understood, whereas an utterance that is poorly formulated from a grammatical point of view, may very well be comprehended as long as it fits the communicative context reasonably well. Indeed, in most situations language users do not pay any attention to the guestion whether their utteran-

12) For an analysis of the concept of fluency, see Sajavaara \& Lehtonen (1978)

13) Obviously these four conditions are not mutually independent, but we need not indulge in a comprehensive conceptual analysis here. 
ces are grammatical or not. Conscious attempts to juage the gramatical acceptability of utterances may sometimes occur, but they are in no way obligatory aspects of utterance production and/or comprehension. Evidently such metalinguistic judgements require an extra effort (cf. Hakes $1980: \mathrm{ch} .2$ ).

Despite all this, the linguist's normal attitude has been to reverse the order of priority for the three properties under discussion. For him the issue of grammaticality is the fundamental question, whereas situational relevance is not considered at all, at least not as long as he is following the traditions of contemplating linguistic constructions, most often sentences, without any situational context at all. This in turn brings us back to the facts that linguists still use written sentences and texts as their subject matter, and that written texts are more autonomous and less context-dependent than most spoken language. For reasons which are largely conventional, and hence not entirely functional in nature, most cultures attach a great deal of importance to the correctness, including the grammaticality, and more generally, to the formal features of written texts. Thus, we see that the linguists preoccupation with grammaticality is closely related to the sequels of the written language bias. 
VI. 4 woras

In traditional linguistics as well as in the layman's understanding of language the word is beyond comparison the basic unit of language. However, worcs are conceived of in two different ways, on the one hand as the buflding-blocks used by the speaker in constructing linguistic products, i.e. as lexical items, and on the other hand as the most important components of actual utterances and sentences, 1.e. as syntactic constituents. It is quite obvious that we are dealing here with two different concepts. I will first comment on the latter.

There are several ways of making the notion of 'word' in the sense of 'syntactic constituent' more precise and workable. The main alternatives are those of 'morphological word' and 'phonological word' (e.g. Ullman 1951:43 ff, Lyons 1968:194 ff). It is rather typical that the morphological word form is the type of unit which is preferred in most linguistic analyses. A morphological word form consists of one or several roots plus their various bound morphemes (affixes etc). (This covers both simplex words and compounds). Now, this notion can of course be at least provisionally defined in structural terms (cf. the notion of 'minimal free form' etc), but it is hardly a mere coincidence that the notion of the morphological word corresponds rather well with the unit of conventional writing, the graphic word, i.e., a sequence of letters surrounded by empty spaces but containing no internal spaces. Thus, at yet another level we see the intimate coupling between the linguist's theory and the layman's views, and between these two and the conventions for correct writing: 


\begin{tabular}{|c|c|c|}
\hline & $\begin{array}{l}\text { unit of linguistic } \\
\text { analysis: }\end{array}$ & $\begin{array}{l}\text { unit of standard } \\
\text { written language: }\end{array}$ \\
\hline phonology & $\begin{array}{l}\text { segment (vowel or } \\
\text { consonant) }\end{array}$ & alphabetic letter \\
\hline morphology & $\begin{array}{l}\text { morphological word } \\
\text { form }\end{array}$ & $\begin{array}{l}\text { graphic word (unit } \\
\text { delimited by empty } \\
\text { spaces) }\end{array}$ \\
\hline syntax & sentence & $\begin{array}{l}\text { graphic sentence } \\
\text { (unit delimited by } \\
\text { major punctuations, } \\
\text { i.e., full stops) }\end{array}$ \\
\hline
\end{tabular}

I am of course aware of the fact that the graphic word is not an arbitrary unit from the point of view of the structure of language (including spoken language). Obviously, it represents a rectification of a unit which by and large is the morphological word. But it may still be true that once the orthographic convention was firmly established, this circumstance served to reinforce the status of the morphological word quite strongly. After all, for spoken language there is another quite plausible candidate available, i.e. the phonological word (phonemic phrase, tone group 14), consisting roughly of a stretch of continuous speech unified by one main stress and an intonational contour. Such a unit normally involves several morphological words; typically function words such as prepositions, articles, weak pronouns and adverbs are enclitically adjoined to a nucleus consisting of one content word (in some cases several such words). If we seriously consider the dynamics of speech behavior, i.e. the processes involved in the production and perception of utterances, it is quite probable that such units are of primary importance. The fact that they have been - intil recently - largely ignored by linguisss is no doubt related to the neglect of prosody (VI.2).

14) I Ignore here any differences between tnese concepts. 
Let me now turn for a while to linguist's conception of lexical items (lexemes). If we consider a broad spectrum of empirical evidence, it will turn out to be necessary to postulate several different types of lexical items, e.g. stems, word forms and phrases (see e.c. sumary in Linell 1979a:ch4). Yet, in modern theoretical linguistics morphemes have been assigned the status of lexical primes; some scholars seem to be prepared to exclude the possibilities of all other types of lexical items. Such a view implies an overly abstract view of language, especially in certain applications of orthodox generative phonology ${ }^{15)}$. By straining our arguments just a little, we might connect this preference for morphemes to the written language bias.

The metalinguistic contemplation of abstract or ephemeral meaning relations underlying many abstract morpheme identities postulated in generative phonology seems typical of someone who is working with words as isolated from the ever changing and perhaps bewildering array of different situational contexts of spoken discourse especially; from a communicative point of view such lexical and morphophonological regularities are hardly primary. Furthermore, although it is sometimes not frankly admitted, such a linguist normally draws quite a lot upon his knowledge of the etymology of the words involved and of the entire history and genetic relationships of the languages under consideration, and this knowledge is ultimately derived from written records, Orthodox generative-phonological analyses (af, fin. 15) tend to recapitulate history in synchronic cescription, as has been pointed out by e.g. Maner (1969).

15) Some extreme examples of orthodox generative-phonological analysis are Chomsky \& Haile 1968, Schane 1968, Foley 1965, Vennemann 1968. For a general critique of the treatment of morphemes as abstract phonological forms, see tinell 1979a: ch 13. 
There is another aspect of the structuralist view of morphology which is worth mentioning in this context, viz. the tendency to regard root morphemes and grammatical morphemes as segment-based expression units of the same kind. This seems natural enough given the orthographic conventions according to which they are represented in basically the same way. However, evidence from studies of utterance production and perception strongly suggests that content words and grammatical morphemes are functionally quite different. For example, data on speech errors and aphasia indicate that most grammatical morphemes should be treated together with other aspects of syntactic construction, e.g. word order (cf.Garrett 1980, Saffran et al 1980, Line11 1982 b). 


\section{VI.5 Tne structure of texts and ciscourse}

A.s part of the immensely increased activities within text inguistics, discourse analysis and conversational analysis in the 1970 's, actempts were of course made to apply linguistic methods of analysis also to larger written texts as well as to spoken dialogues. Linguists started to search for the structure of texts and conversations. Some of these efforts ended in disappointment; one did not find any good examples of wellorganized structure, particularly not in certain forms of spoken discourse. However, the main reason for this may well have been that most scholars started with a preconceived notion of what kind of structure they expected to find. They zried to use a normative concept of structure, one which was based on rules for how written texts should be composed and organized. Both outsiders and insiders in text linguistics now seem inclined to agree that the attempts to set up generative text grammars (van Dijk 1972, Thorndyke 1975 and others) were failures. It was useless to try to define possible vs impossible texts in analogy with grammatical vs ungrammatical sentences. (In fact, even the latter task is dubious when applied to natural spoken language, as we have seen (VI.3)). Halliday \& Hasan (1976) adaress the issue in this way:

'A text is a unit of language in use. It is not a grammatical unit, like a clause or a sentence; and it is not defined by its size. A text is sometimes envisaged to be some kind of super-sentence, a grammatical unit that is larger than a sentence but is related to a sentence in the same way that a sentence is related to a clause, a ciause to a group and so on: Dy constituency, the composition of larger units out of smaller ones. But this is misleading. A text is not something that is like a sentence, only bigger; it is something that differs from a sentence in kind.... A text does not consist of sentences; it is zealized by, or encoded 12 , sentences. If we understand $i t$ in this way, we shali not expect to find the same kind of structural integration among the parts of a text as we finc among the parts of a sentence or clause. The unity of a text is a unity of a different kind.' (Ha111day and Hasan 1976:1-2) 
The upshot of the argument is that texts and discourses do not have grammatical structures (Iike sentences and smaller units), and texts cannot be assessed with regard to grammaticality. Instead, texts convey meaning in contexts, and what might be called discourse structure should be explained with reference to the dynamics of the whole communication situation. to the processes of production and comprehension which can hardly be treated adequately without recourse to the intentions, expectations and partially shared worlds of the communicating parties (Levy 1979). But the linguistic approach, which was criticized earlier, simply followed the tradition of studying language as products, as static objects, which are searched for a structure of well-defined internal relations. 
VII, SEMANTICS

\section{VII.1 Introduction: The autonomy of linguistic meaning}

The typical attitude prevaling in most of modern theoretical linguistics is that language can be studied as an autonomous structure without any direct consideration of conditions of use. This applies most straightforwardly to the outer form, the expression side, of language (V.4), but many scholars treat meaning, the content side, in the same way. In short, meanings are held to be

- objective, i.e. they are not dependent on the ways any given persons happen to understand them,

- autonomous and disembodied, i.e. they can and should be treated as independent of what human beings do in speaking, understanding, and acting,

- compositional, i.e. they are abstract objects with well-defined inherent properties, and can be analyzed in terms of components, i.e. "smaller" objects (more "primitive" concepts and the like). (Lakoff \& Johnson 1980).

Furthermore, it is assumed that words, sentences, texts, and discourses have meanings "in themselves", as linguistic objects as it is often put. They are said to "carry meanings" with them (IX.1). The meaning of a given linguistic object can be unearthed by means of a sophisticated linguistic analysis which aims at finding the correct interpretation or the semantic representation inherent in it (note the singular forms which are used here) (cE V.5).

The view just sketched is utterly misconceived, at least if we are incerested in the meanings or interpretations relevant in actual communication, or, for that matter, in chinking. The interpretation of an utterance, a discourse or a text is never (I will argue) completely inferrable from the linguistic objects alone. It is not inherent in the Iinguistic signs (the "soundmeaning correspondences") taken as situation-independent enti- 
ties. In practice, there is always a need for different kinds of background knowledge, i,e, knowledge which is extrinsic to language but usually more or less avałlable to senders and receivers in communication. Consider (1) as a simple example:

(1) He is dangerous.

Such a sentence can never be understood without several types of background knowledge. The listener (or reader) must be able to tackle a number of questions whose answers are in no way inherent in the semantic representation of the sentence as such (however this is conceived): Who is he? (referential specification; note that this is still necessary, even if we use a common noun (that man) or a proper namne (Ronald Reagan) instead of a pronoun). In which way, to what extent, is he dangerous? To whom and what, when and where is he a threat? ldetermination of standards of comparison and precision, referential specification, intensional precisation, elimination of vagueness and ambiguity). Who uttered (1), when did he (or she) do it, and why? (assignment of intentions and reasons to the sender). These latter questions are concerned with the so-called why of communication (Ducrot 1972) which is always relevant for the addressee. A resonable situational interpretation ${ }^{1)}$ can only be the result of a complex interplay between various situation-specific factors and the inherent meaning potentials (Rommetveit \& Blakar, 1978) of the inguistic units and constructions. The sontribution of the linguistic expressions with their meanings is often very important but it is never enough by itself. Instead, words and utterances should be seen as clues to interpretation, as instructions to the listener to search for an interpretation along certain lines lc E Gardiner 1951, tinell forthc.). Utterances allow for, or point to, certain interpretations, but they do not exoress or transter them (IX.1). Accoraingly, Merleau-ponty (1962) speaks of "the essential allusiveness and incompleteness of speech" as

1) More about this notion in \$ VII. 5 . 
a ubiquitous and fundamental feature of all linguistic communication; it is not restricted to "special forms of insinuations, half-sayings, allusions to little tales of an intentionally nonserious character" (Vološinov 1973:97) and similar uses of language.

In discussing formal and semantic autonomy (III.1) I referred to Olson's (1977) pertinent analysis of the written language bias in linguistics and related disciplines. However, even Olson argues that written texts are, or may be, semantically autonomous. We recall that for him this was the most fundamental difference between writing and speech. But such a view can hardly be true in a strict sense. It is a fact that written texts are not semantically autonomous either (cf VII.6). However, they are relatively more autonomous than spoken dialogues, and this is a circumstance which has not failed to exert a great influence on linguists ${ }^{-}$views of semantics. 
VII.2, word meanings

The view that there are immanent meanings, or interpretations inherent in the linguistic signs as such, i,e. associated with the Iinguistic expressions (sign vehicles), has been much cherished by linguists. Sometimes these meanings are talked about as 'literal meanings', and one should carefully note the word 'literal' here! The assumption of literal meanings pertains to several linguistic levels. Here we will consider word meaning first and return to sentence meaning later on (VII .5).

Within linguistic semantics there have been several theories of word meaning, and not all of them are equally amenable to characterizations of the kind that I am going to suggest. However, cne element that seems to be implicit in many theories is the idea that many ('literal') uses or interpretations of a given word can be directly inferred from an underlying invariant word meaning. (Some would put it even stronger saying that the literal interpretation is identical to the word meaning). Such a conception is compatible with theories based on Grundbedeutung as well as Gesamtbedeutung.

The standard view is that word meanings are definite, fixed and stable, e.g., a set of invariant semantic features. These meanings are ready-made, they exist "out there" in the language (la langue) as a system, and as such they are available for use by speaker-listeners (writer-readers). An extreme view is held by structuralists who maintain that word meanings should be defined entirely within the system itself without any resourse to extralinguistic experience. According to this view, the meaning of a given word oonsists in being different from other words; dans la langue il $\mathrm{n}^{*} \mathrm{y}$ a que des diffërences (Saussure 1964:166).

Instead of construing word meaning in terms of sets of invariant semantic feature complexes (as, for example, in the semantics of a generative grammar) one could look upon the meaning of a given word as something which is "dynamic, only partialiy determined" and "open and susceptible to contextual modifications". 
"In communication when what is initially intersubjectively shared becomes expanded and/or modified" (Rommetveit \& Blakar 1978:354). In Rommetveit's view the word has the potential of eliciting different semantic operations which are partly different in different situations and therefore result in aistinct situationspecific interpretations. Another way to put it would be to say that word meanings place certain conditions on possible situational interpretations, or that they point to, allude to, certain interpretations.

Vološinov (1973) arques that the conventional view on word meaning derives from the traditional work by linguists and philologists on written texts in foreign languages ("the grandiose organizing role of the alien word"), which necessitated the development of dictionaries with their standarc definitions of word meanings. Obviously, it makes a great difference if someone is acquiring his mother tongue in naturalistic situations, which may be assumed to lead to an implicit recognition of the negotiability and context-sensitivity of meanings, or if he is learning a foreign language by consulting dictionaries as a means of decoding written texts. In general, the system-internal definition of meanings in terms of semantic features or the like seems to fit the latter predicament, i.e, the learning of foreign and learned words and concepts, rather than the acquisition of everyday concepts. Learned vocabulary is typically acquired from written texts and/or by verbal definitions, i,e., the whole process is essentially language-internal.

The conception of word meaning as something zixed and stable recurs in several contexts. It often seems to be presupposed that understanding what a word means simply consists in knowing its linguistic meaning or being able to provide an explicit verbal definition. We are therefore often faced with an all-or-nothing conception; either someone understands what a word means, or else he does not do so. Something like this seems to be implicit in the theory of a linguist who performs investigations of people's knowledge of words by simply testing the uncerstanding of 
isolated sentences in which the words in question occur. Another case would the parent who observes his little child reacting adequately (or not adequately) to the use of a certain word in a given situation, and then declares that the child understands (or does not understand) the word tout court. This is obviously absurd since the same word can often be understood in many ways and at different levels; we could talk about listeners"varying depth of understanding in analogy with the speakers" depth of intention" (Naess 1953). Therefore, one may perfectly well understand a given word in one situation, where, for example it is perhaps supported by other factors in a fortunate fashion, and at the same time completely fail to understand it in another situation.

In this discussion I have alluded to the layman's theory that "each word has its own proper meaning". It might be pointed out that this "theory" exists in a reversed variant too; it is assumed that "each thing has its right name". The view that there is one unique way of expressing something precisely to the point ("le mot juste") is the other side of the coin (cf Gardiner 1951:174). 


\section{VII.3, The boundary between semantics and pragmatics}

One of the most hot $1 y$ debated issues in the current theoretical discussion within linguistics concerns the relationship between semantics and pragmatics. Is it possible and/or desirable to split up the study of meaning into two disciplines? This is a complicated problem which is currently being highlighted in a great number of books and articles. For obvious reasons I am unable to discuss here all the pros and cons. Rather what is at stake here is simply the fact that a distinction has been made by many scholars. The dividing line can be crawn in different ways and at different places, and I will mention three different alternatives in the sequel (for further discussion, see Aliwood 1981).

I will not argue that the distinction between semantics and pragmatics in any of the three versions or the theory to be discussed here corresponds to, say, what is inherent in the semantic representation of a written text (cf. Olson's hypothesis that texts are semantically autonomous, VII.1) vs what additional odd factors are involved in the understanding of spoken utterances. There is no such simple relationship between writing and speech on the one hand and semantics and pragmatics on the other. Furthermore, the distinction has never (to the best of my knowledge) been rationalized by linguists on any grounds of that kind. On the other hand, this also applies to almost all the other theoretical issues discussed in this book; Iinguists generally argue that the theoreticai options they take have a clear basis in the linquistic phencmena themselves rather than simply in the tradition of analyzing only writzen language.

As far as the semantics-pragmatics distinction is concerned, some scholars tend to rationalize it on the grounds that it helps the linguist to delimit language properly. Many linguists have been quite anxious to establish and malntain linguistics as an independent science distinct from, say, psychology, socio- 
logy and philosophy. Since one suspects that the actual understanding of utterances and texts on the part of listeners and readers is more or less dependent on their encyclopedic knowledge of the world, and since most linguists would prefer to have a neat dividing line between their (hopefully) well structured science and the whole mess of encyclopedia, the attempt to delineate a more restricted study of meaning, that of semantics, may be a sensible strategy. After all, in comparison with pragmatics, semantics is (in most people's opinions) more close1y integrated with the language system itself (la langue, competence).

However, no matter what the explicitly given or consciously admitted reasons for setting up the semantics - pragmatics distinction are, the written language bias is undoubtedly there as a general background, and it may very well be treated as a contributing cause. This can be argued in all the three cases which I will now advert to ${ }^{2)}$.

The first, and perhaps most popular, theory (e.g. Lyons 1977: 591) would define semantics as the study of those context-independent meaning elements which are tied to the linguistic expressions, i.e., part of the linguistic signs as such (signs taken roughly in saussure s sense), and are (considered to be) invariant across all situations of use. Pragmatics, on the other hand, would then deal with the specifying effects of various situational factors, and try to account for the meaning of specific utterance tokens. In other words, semantics would fo-

2) These three attempts to legitimize the distinction between semantics and pragmatics are all important, but I do not claim that they exhaust the set of possibilities of drawing the boundary line. See for some discussion A.llwood (1981). 
cus on those contributions to the situational interpretations ${ }^{3}$ of utterances and texts which are provided by the language itself (the rules for using words and expressions), while pragmatics would encompass the whole process of determining situational interpretations, the entire interplay of all the various linguistic and extralinguistic factors (cf.II). Accordingly, semantics and pragmatics could be seen as concerned with the meanings of linquistic expressions considered as types and tokens, respectively ${ }^{4}$.

It might be noted in passing that my own theoretical preferences (cf. the distinction between linguistic meaning and situational interpretation, VII:5) would, by and large, coincide with these views, although I am aware that it may be difficult to defend a strong version of such a theory. Clearly, there are some meaning potentials associated in the user's mind with the linguistic expressions as types. Thus, even a sentence like He is dangerous, which - as an abstract sentence type - is "unspecified" in obvious ways (VII:1), can be presented in abstracto, i.e. without any observable supporting context, and most of us would still be able to (re)construct at least some ingredients of a possible interpretation. We would understand what could or must be the case in a situation in order for the sentence to be meaningfully used there ${ }^{5}$ ).

3) For this term, see VII:5.

4) A somewhat different variant of this theory would involve the claim that semantics deals with conventional meaning, and pragmatics with nonconventional (natural) principles for assigning interpretations (of. e.g. Grice 1975, Sadock 1979).

5) Many semanticists would prefer to talk of truth conditions (VII:4). Accordingly, the linguistic meaning of a sentence type would define the conditions that situations must fulfil in order for the sentence to be true of them. 
Whatever the merits of this first-mentioned attempt at distinguishing semantics from pragmatics are, it is not too farfetched to suggest a possible link between this and the written language bias in linguistics and philosophy. The idea of context-independent, invariant meaning features belonging to the language itself is something which gets easily promoted through the traditional linguistic study of written sentences and texts; I have already pointed to the relative autonomy and explicitness of texts as compared with most spoken dialogues (II, VII.I). Here is Vološinov again:

"The isolated, finished, monologic utterance, divorced from its verbal and actual context and standing open not to any possible sort of active response but to passive understanding on the part of a philologist - that is the ultimate "donnee" and the starting point of linguistic thought".

(Vološinov 1973:73)

The second theory has also been hinted at, e.g. by Lyons (1977: 591). It is based on the different communicative functions of utterances and texts, and on the linguist's assessment of these functions in terms of importance and priority (cf. IX.2, and also VII.4). Thus, it may be suggested that semantics be exclusively concerned with those meaning elements that have referential or cognitive functions, whereas the study of all the various social, emotive, practical and associative aspects be relegated to pragmatics ("the pragmatic waste-basket"). Such a conception is also in keeping with the written language bias, since writing clearly enhances the referential and cognitive aspects, the so-called intellectual functions, at the cost of the other functions. This definition of semantics would also fit the nlew that there is a close connection between semantics and logic (cf. VII.4, VII.9). Steluer comments on the actempts to create a universal logic with a Eixed semantics fn the following way:

"The slippery, ambiguous, altering, subconscious or traditiohal contextual reflexes of spoken language, the centres of meaning which Ogden and Richards termed "emotive" and which Empson treats under the rubric of 'value' and 'feel', fall outside the tight but exiguous mesh of logic. They belong to the pragmatic". 
The thirc theory ties up with the distinction between normative and cescriptive studies, which was applied to semantics by Carnap. According to this view, semantics is normative; it studies meanings and interpretations as they should be, or, alternatively, as they would be if certain well-defined rules were consistently followed. This is Carnap"s "pure semantics", a discipline which may be seen as a subpart of logic. Pragmatics, on the other hand, would be the descriptive study of how language is actually used; how concepts are in fact applied to different situations, and what interpretations are arrived at by real language users. This is close to what Naess (1953) termed "empirical semantics". Even this third theory may be associated with the written language bias.

The written language is intimately connected with norms of language and training in an allegedly correct and logical use of language. The pursuit of normative semantics is therefore a rather natural endeavor for someone dealing with the theory of correct (written) language. 
VII.4, Semantics and truth

"If we knew what it would be for a given sentence to be found true then we would know what its meaning is".

(Carnap 1953)

Linguists and philosophers like to theorize about the functions and goals of semantics in a way that links it up with questions of the theory of truth and truth conditions.

"Despite the uniquely fragmented state of contemporary linguistic theory at the present time, there is a remarkable consensus with respect to one fundamental issue: the nature of semantic representation. Scholars as disparate as Montaque, Chomsky, McCawley, Katz, Sadook and Partee would, I think, now agree that the semantic representation of a sentence is a representation which expresses the logical form of the sentence, or, put differentiy, a representation which expresses the conditions under which that sentence would be true".

(Gazdar 1980:5)

It is significant, especially in the light of the discussion in VII.3, that Gazdar adds to these statements that "other nontruth conditional aspects of the meaning of the sentence belong to pragmatics".

In logic, which may be seen as a special development of sentence semantics, truth and truth conditions have always been of primary importance. How should one characterize truth vs falsity? What are the conditions to be fulfilled if a given statement is to be true or false? In modern linguistics, truth conditions have also played a major role, as Gazdar notes. As an illustrative example indicating the primacy of truth conditions in the linguists' conception of meaning we may fecall ?artee's (1971) ciscussion of the respects in which transformations in a standard generative grammar) are, or could be said to be, "meaning preserving"; the proposal given there was that transformations must not change truth values.

A truth-conditional semantics is thus concerned with (mainly or only) the referential ('intellectual') function of language, 
i.e. aspects of meaning which are emphasized in written cexts. The referential-cognitive aspects of language are clearly more independent of the particular contexts of use, more independent of specific senders and receivers, than are the other aspects (emotive, evocative, expressive, social etc, cf IX.2). The referential association can be depicted as an abstract connection between language and the world:

linguistic expression (e.g. a statement) with its semantic representation (a set of truth conditions)

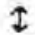
things referred to in the "real worla"

The relation here designated by an arrow $(\longleftrightarrow)$ can be characterized in terms of truth vs falsity; either the statement corresponds with reality, or it does not. There is, in other words, a close connection between a correspondence theory of truth, and the conception of linguistic meaning as truth-conditional in nature.

The goal of describing and analyzing objective reality in a true manner is of course rather strongly stressed in certain verieties of mainly written discourse, in particular scientific texts. But there are many other uses of language, especially in spoken dialogues, where the purely referential elements are not of primary importance, and where the truth of the statement is not the main thing. If we take a simple utterance like It is raining, there are surely many situations of use, where the determination of the truth of this statement is not central to the speaker's intention or to the listener's actual interpretation. Most often the Ifstener would take it for granced that the speaker provides him with a veridical description. Rather than checking whether the speaker's assertion is actually true, the listener asks himself more important questions: What is the point of this assertion? For what purpose does the speaker utter it? In what way should I utilize this piece of information? That is, the why of communication is of utmost importance, and this can only clumsily (if at ali) be accounted for in terms of truth conditions. 
Another case in point which shows that the question of truth is often downgraded in actual discourse concerns the normal use of referring expressions (noun phrases). If I say that man with a camera is dangerous, the point of using the referring noun phrase (that man with the camera) is not that of issuing a statement, the truth of which the listener should determine, but simply that of using a linguistic expression such that the listener succeeds in identifying the referent that $I$ as speaker have in mind. In fact, the act of reference may be successful even if, on a closer look, either the speaker or the listener, or both, discover that the man was after all not carrying a camera but a pair of binoculars (cf. Donnellan 1971). Thus, the order of priority is this: the superordinate goal is the evocation of a certain act of understanding in the listener (he must understand what the speaker means by his utterance), a means to attain this goal is through reference to a certain person, and this in turn involves the subordinated tactic of describing this person in a certain, veridical way.

Current linguistic semantics thus overrates the importance of truth. The concentration on truth conditions turns into absurdity if it is argued that this is the only significant aspect of meaning. Steiner (1975:211 ff) has attacked this concentration on truth in linguistic semantics and stressed the importance of our ability to imagine and describe things as they are not. The fundamental properties of language, or rather linguistic communication, are incompleteness, vaqueness and allusiveness (cf.Merleau-Ponty); the whole truth is never conveyed, it is only indicated or alluded to. Thanks to this, we can use language creativeiy in an ever changing social and physical reality, and we can, if we must, lie or tell half-truths, and this may aven be a precondition for survival in some situations (Steiner, op cit). 


\section{VII,5, Speech acts, sentence meaning and utterance meaning}

Some semanticists (e.g. Grice 1957) have tried to distinguish between sentence meaning and utterance meaning. The former would be some type of invariant meaning which pertains to the linguistic expression as such i.e. Without any consideration of particular situations where it may be used (cf. VII.3). After a11, most linguists would surely agree with Lyons (1977:35) that "a sentence like "It's raining" has a certain constancy of meaning irrespective of the purpose of the communicative act in which it is used". I would here prefer the term linguistic or structural meaning, since the linguistic expression involved need not formally be a sentence (cf. VI.1). This must be carefully distinguished from the situational interpretations of particular occurrences of the expression (e.g. It's raining), i.e. interpretations made when the expression is used in different specific utterances in particular situations. The term 'situational interpretation', which I use instead of utterance meaning, is applicable to non-verbal acts as well, and is, moreover, meant to imply that the interpretation pertains to a comprehensive communicative act, in which the verbal utterance is just one (albeit important) part. As will be pointed out presently (VII.6), there are always several possible situational interpretations of the same utterance token. For example, we must distinguish between how a speaker wants his utterance to be understood, and what the listener actually understands.

What then is the proper relation between linguistic meaning and situational interpretation? The complications of this issue can haraly be unravelled here, but it is still possible to contrast two basic theories.

The first type of theory, which has gained a considerable popularity anong linguists at least since Searle (1969), implies that there are two kinds of communicative acts, one in which the situational interpretation, what is or should be understood, is simply and precisely equal to the linguistic meaning (this 
would then be the "literal" meaning or interpretation), and one more complex type in which the understanding involves something more; the speaker does not mean "exactly what he says", and hence we must proceed to a "non-literal" or "figurative" interpretation (indirect requests, metaphors, jokes, irony, sarcasm, and many other implicatures). Thus, this dichotomy corresponds to Searle's distinction between direct and indirect speech acts.

Searle"s theory of direct speech acts is based on the thesis of semantic autonomy; it is assumed that there are cases where the task of the listener is "just" that of understanding, or "reconstructing", the structural meaning, usually called the "semantic representation", of the linguistic expression used. Searle also formulates this as the "principle of expressibility": "whatever can be meant can also be said and understood" (1969: 19-20). Thus, it is argued that fixed and finite meanings can (in fortunate cases) be converted into exact expressions, and vice versa. In other words, direct speech acts would be such communicative acts, where the speaker "says exactly what he means". I would deny that such acts exist. In fact, considering what has been said in $\S$ VII.1, it is remarkable that such a theory has been proposed at a11. On the other hand, it is not entirely surprising given the written language bias in linguistics and logic (cf VII.7). After all, contemporary linguists are accustomed to analyzing sentences in abstracto, thereby figuring out what "their semantic representations" are.

The other type of theory, which seems to me much more plausible, says that the understanding of an utterance always involves something more and something beyond constructing the linguistic meaning. Thus, for example, the why of communication is always more or less important, and this is not something which can be directly or exclusively associated with the linguistic expression or the overt behavior as such. Other situational factors also intervene (see VII.1). Even if we understand a sentence seemingly in vacuo, e.g, if we reflect on the meaning of sentence like tie is cangerous or It's raining without any given 
extralinguistic context - which linguists are used to doing (cf above) -, we take part in a language game with special presuppositions and expectations anc cannot avoid constructing at least some rudimentary aspects of an imaginary context. The same applies to written texts (cf VII, G). Thus, Rumelhart is justified in saying:

"In sumary then, the supposition that conveyed meanings are ever identical to literal meanings (where literal meanings are assumed to be those given by a compositional semantic theory) is surely suspect. The problems of determining conveyed meanings of literal sentences are no less difficult ( $I$ believe) than finding those of figurative ones".

(Rumelhart 1979:86)

In concluding this section I should point out that even those who believe in the existence of direct speech acts usually concede that the literal meaning of a sentence comprises only "a set of truth conditions", and that these have to be used "against a background of assumptions that are not explicitly realized in the semantic structure of the sentence" (Searle 1979:95). What searle apparently alludes to here is assumptions concerning the identity of referents, universes of reference and comparison (e.g. the standard of comparison and precision applied when sentences like the box is big and France is hexagonal are used) etc. It is indeed remarkable that somebody can admit this and at the same time claim that there are cases (of so-called direct speech acts) when "Iiteral sentence meaning and speaker's utterance meaning are the same" (Searle, op.cit. :96). Moreover, apart from this whole complex of referential specification, we must - as I pointed out earlier - add the immensely important aspect of the "why of communication" to the list of items that are ustally ignored in linguistic semantics. 
VII,6 The interpretation of utterances: The roles of the speaker, the listener, and the outside observer.

It is commonplace that linguists talk about the (semantic) interpretation of a given utterance, discourse or text. However, this unique, "correct" interpretation is a fiction. There are always many - in principle infinitely many - interpretations of a given utterance available. (It is an entirely different matter that most of these are never computed or attended to in the concrete communicative situations). For one thing, we may distinguish between the speaker's, the listener's and an outside observer's interpretations. Furthermore, in neither of these cases is there any single "correct" situational interpretation.

First of all, it is usually not the case that the speaker has a single intention in mind; there are often many motives, reasons, desires and intentions behind a speaker's utterances. Some of these may be partly contradictory, they vary a lot as regards importance, consciousness etc, and some of them are recognized by the speaker only after the utterance has been produced. The proper interpretation of an utterance is often negotiable, and the listener may often convince the speaker afterwards that a certain interpretation of his utterance is reasonable although it was never (consciously) intended by him.

Similarly, the listener's interpretations are relative to many factors, such as his background knowledge, his intelifgence and imaginative power, his degree of attention and interest, his social relations to the speaker and hts actual social interaction with the latter, his willingness to think over what has been said and what follows from that. We all know that one single utterance may occasionally give rise to a long - in principle infinite - series of conclusions, associations and other reactions on the part of the listener. There is no non-arbitrary point where this process of understanding of a given utterance stops and some other activities take over. Also if we disregard the off-Iine reflection occurring after and outside the inter- 
action 1tself (afterthoughts etc), and only consider the direct on-line understanding of what is part of the social interaction in the communication situation, we must admit that there is a wide range of possible situational interpretations on the part of the listener.

The more or less "objective" observer, who analyzes a dialogue from outside, naturally does so from a rather different point of view. He is not directly engaged in the social interaction, and is therefore detached from a number of ties, often emotive in character, which hamper certain interpretations on the part of the parties involved in communication. Speakers and listeners have seldom "time or mental capacity to contemplate past behavior, monitor present behavior, and plan future behavior" (Storms 1973:166), and hence they are likely to remain ignorant of many meanings and interpretations that could be attributed to their behavior. It also appears that, in many situations, a given speaker $A$ is more prone to explaining his own behavior by reference to situational factors than is his listener-adaressee $B$, who, in trying to explain the same phenomenon, i.e. A's behavior, may assign more importance to $A^{\prime} s$ dispositional properties as causal factors. This may be due to differences in information about the event, behavior, and context that is available to the speaker as opposed to the listener, and to differences in how this information is processed (Jones \& Nisbett 1971). After all, an actor, e.g. a speaker cannot freely observe himself while acting, and there may also be motivational reasons for avoiding too much self-observation (Storms 1973)

In all these respects, an outside observer is of course much less constrained than both communcating parties; he can explore the whole situation (especially if he has access to videotapes) and exploit his background knowledge (perhaps a vast scholarly expertise). On the other hand, he lacks first-hand knowledce of the actors actual motives, thoughts and feelings, and may therefore at times misrepresent their intentions and 
expectations. In addition, it is important to realise that observers are also dependent on differences in background knowledge, interest, willingness to consider different alternatives etc, and therefore we will get a broad spectrum of possible interpretations also on their part.

Linguists seldom find it worth while discussing the multiplicity of situational interpretations of texis and discourses. It is often left unclear whose point-of-view, the speaker's, the listener's, or the observer's, is applied, when meanings and interpretations are assigned and analyzed by linguists (psychologists, anthropologists etc). (This is not to say that it is always an easy task to disentangle the various possible and/or actually occurring interpretations of particular discourses, complexly intertwined as they are). In practice, however, the interpretations are of course mainly those of the expert-observer, i.e., the analyst himself. This specialist (linguist, psychologist, or whoever) must of course have a very profound knowledge of the language and the culture he is analyzing. He must be a native speaker, or, at least, work in close cooperation with native informants (cf. also the participant-observer role in social anthropology). As we noted above, the expertobserver's role is very different from that of a speaker or a listener actively engaged in a social interaction. Yet his assessments are hardly divine, they are not objective in an absolute sense, since they are constrained by particular theories and attitudes, some of which are dependent on specific academic traditions and schools, while others are perhaps more generally typical of the socio-cultural class to which academics belong. In a way the expert-observer may be regarded as a specific type of receiver-interpreter acting under very special conditions. Yet we often regard our own assessments of gramaticality, meaning and meaningfulness etc as the only correct, as-it-were objective evaluations possible (as if they uncover the true essence of language). We tend to regard the primary interpretations made by real speakers and listeners as uninteresting, affected as they are by various distractions, memory limitations, 
accidental situation-specific needs and impulses. This is, in my view, unfortunate, since we need both empirical studies of how meanings are created and assigned in actual discourse and analyses performed by outside expert-observers. What some of us do, instead, is to regard the expert-observer's purportedly correct interpretations as ideal, as something which should be attributed to "ideal speaker-listeners", i.e., those imaginary actors who live "in a completely homogeneous speech-community" and who"know" their "language perfectly" and are "unaffected by such grammatically irrelevant conditions as memory limitations, distractions, shifts of attention and interest, and errors (random or characteristic) in applying (their) knowledge of the language in actual performance" (Chomsky 1965:3) 6).

It would be absurd to argue that the three perspectives of the speaker, the listener, and the observer could be equated, when it comes to the analysis of yerbal communication in face-toface interaction. Note, however, that it is a little less absurd to do so, if we concentrate on the interpretation of written texts. There the reader is much less constrained by time limitations, and the social pressure of other people, including the sender, is much less obvious. The reader can rely on longtime reflection and make extensive use of his encyclopedic knowledge and the various types of expertise that he happens to possess. Therefore, the distinction between the receiveraddressee and the outside observer becomes much less clear. The image of the expert-analyst as a generalized ideal reader turns out to be, if not always fruitful and applicable, so at least sometimes a reasonable first approximation. It is, therefore, understandable that linguists dealing with written language and

6) I quote Chomsky (1965) here in Eull cognizance of the fact that he is not concerned with hypotheses and claims about situational interpretations of utterances. 
written texts have not felt any strong need to make a clear distinction between what the expert-observer does and what ordinary readers do (or should do). This automatically leads us over to the practice of literary analysis and text interpretation, which I will discuss in the following paragraph. 


\title{
VII.7. The interpretation of written texts
}

\author{
'Es liegt alles am Wort' (Martin Luther)
}

In the preceding section I argued against the theory that there is a subclass of speech acts, for which it is possible to assign more or less directly or automaticaliy a unique 'literal' interpretation ('direct speech acts'). I will now advert for a while to written texts and make essentially the same point there. Although written texts are relatively more autonomous as far as the semantic content is concerned, it can never be true that the whole interpretation may inhere in the text itself. One must always draw upon extratextual factors, something which is now becoming more widely recognized among text linguists (cf. e.g. de Beaugrande \& Dressier 1981).

The thesis of semantic autonomy shows up not only within linguistics proper, but sometimes also in the theory of literature. Some scholars apparently favor a view that there is, at least in some cases, one unique proper interpretation of a given piece of literature, say a poem. The standard view is then that the correct interpretation is one which experts agree upon after having analyzed the text meticulously and with due attention to all the features in it. The text is thus treated as an autonomous linguistic structure, the understanding of which demands no more and no less than a proper linguistic for perhaps philological) exegesis.

It should be noted here that there is an alternative conception of the unique and correct interpretation. According to this latter theory, the right interpretation is simply the one that the author intenced. However, we have already seen that speakers often post hoc admit that their utterances can be ascribed reasonable interpretations that they never intended them to have, and the same thing applies to writers and texts. The theory under discussion is therefore fraught with difficulties. Accordingly, it is often dismissed with the following argument. 
If the poet did not succed in expressing what he had intended, then his intentions (whatever they were) are uninteresting so far as the reader's analysis isconcerned; the only thing that counts is what the author has actually written.

However, the unique correct interpretation in the sense of something intersubjectively shared among expert observers is also a fiction. There is no such thing as the only (correct) situational interpretation. There are lots of interpretations which are more or less justifiable, depending on what kinds of background knowledge the interpreters have but also on such things as their reading strategies, intelligence, imagination, willingness to pursue their interpretive activities for a sufficiently long time etc. Note, however, that the theory of the correct interpretation often serves to turn literary analysis into a normative rather than a descriptive discipline; this theory does not encourage the scholar to investigate what kinds of interpretations people actually make of a given piece of literature.

The quotation used as a vignette for this section is intended as a reminder that the thesis of a unique and correct interprezation in the text was vigorously propounded by Marin Luther (see III.1). Luther argued that the proper interpretation of the Holy Scripture was to be found in the text itself, in 'God's pure words'. In his opinion the bible was far from being obscure or ambiguous; on the contrary it was a clear and unambiguous message which could be decoded by anyone possessing the necessary knowledge of the language. Here was an enormously strong impetus for doing linguistic analysis and philological exegesis. Form and content (in the sense of (situational) interpretation) were directly coupled. Therefore, the study of language and the understanding of the gospels were inseparable activities. 


\section{VII.8, Loglc anã formal languages}

The idea of the semantically autonomous text is most clearly and radically reflected in the traditional conception of logic and of what is required from a logical argumentation. The language of formal logic is a regimentation of certain forms of written language. In the light of what was reviewed in III.I it seems unbelievable that logic could have been developed at all in the absence of written language.

The symbols and symbol combinations of formal logic are supposed to have exact semantic interpretations, which must be mechanically computable without recourse to any "extratextual" information. Accordingly, in order to perform successfully on tasks of logical argumentation one must be able to draw the right conclusions using only the explicitly given lingulstic formulation and its supposedly unambiguous, exact semantic interpretation. One must not ask oneself whether the task squares with one's experience of everyday reality, whether the conclusions make sense in real life situations. However, this is exactly what ordinary people tend to do when faced with logical tasks. This of course holds most clearly for children, who are very dependent on their experience from real life contexts?' It is wellknown that Piagetian logical tasks may well be solved by quite young children, if the problems are applied to subject matters which the children are familiar with, whereas they cannot be solved by much older children if they are couched in abstract symbols (Wason 3 Johnson-Laird 1972, Donaldson 1978, Rommetveit 3 Blakar 1978). The same applies to normal aduits, as olson (1972) points out in his review of some studies:

7) The same seems to apply to nonschooled individuals in iliterate cultures. Schribner (1977) summarizes several cross-cultural studies on verbal reasoning, and points out the consistent differences between schooled and nonschooled subjects (i.e. subjects acquainted vs non-acquainted with written language and the premises of written communication) (from several mutually unrelated cultures in Africa, America and Central Asia) with respect to their performance on logical tasks (solving syllogisms). Nonschooled subjects base their judgments on their own everyday experience of factual matters (i.e. matters having to do with the content of the syllogisms), whereas schooled subjects are much better at basing their conclusions exclusively on what is explicitly stated in the premises of the syllogisms. 


\begin{abstract}
"Formal reasoning has led to a reliance, where possible, on the use of symbols related by a logical calculus. To illustrate the difficulties, I will use three studies from our laboratory. Bracewell (Note 2) has shown that the simple propositional statement employed by Wason and Johnson-Laird (1970), "If p is on one side, then q is on the other," is ambiguous in at least two ways: "one side" may be interpreted as referring to "the showing side" or to "either the showing side or the hidden side"; "if...then" may be interpreted as a conditional relation or as a biconditional relation. Differences in subjects ${ }^{-}$performance can be traced to different interpretations of the proposition. In a similar vein. Hidi (Note 3 ) has shown that if a simple proposition such as "if you go to Ottawa, you must travel by car" is understood as describing a temporal event, subjects draw quite different inferences than if it is treated purely as a logical statement. In a developmental study, Ford (1976) has shown that, given a disjunctive statement, children (and adults in natural language contexts) treat "or" as posing a simple choice between mutually exclusive, disjoint alternatives (for example, "Do you want an apple or an orange?" "An apple.") When children of five or six years of age are presented with "or" commands involving disjoint events as well as overlapping and inclusive events - the latter being involved in Piaget's famous task "Are there more rabbits or animals?" - Ford found that children's logical competencebreaks down only when the known structure of events runs counter to the presuppositions of the language. Rather than revise their conception of events - rabbits and andmals are not disjoint classes - children misinterpret or reject the sentence. They say, for example, "There are more rabbits because there are only two ducks!" "
\end{abstract}

(01son 1977:274)

Stenlund (1980) has subjected the ideas behind formal logic and analytic philosophy to a penetrating and very interesting analysis, which leads up to a rather negative assessment of their "world view". His subject matter is thus the development of logic and mathematics into pure "non-empirical" sciences, disconnected from their practical applications and from their origin in real life problems. In particular, Stenlund's objective is to show that analytic philosophy and logic depicts the world as made up of objects (cé. I). Here are a couple of quotations:

"They (i.e. logicians/PL) structure language in the same way as they imagine the logical form of the world: in quantitative partwholes. They think as they speak: in outer forms, in writing. 
They think in terms of writing - begriffsschriftilch' lop.cic.: 99).

'Logics as a phenomenon, as that which rests upon the separation between content and form, between living and thinking, is perhaps that subject which better than anything else brings out the "true nature" of object thinking' (op.cit.:116).

Stenlund argues that Frege's logic in terms of arguments and functions blurs a number of distinctions that are important in natural logic; for example, many of Aristotle's categories are collapsed (cf Stenlund, op.cit. : 146)

8) As a simple case in point we could here draw an analogy with the linguists'now popular conceptions of semantic structure, according to which such a fundamental distinction as that between attribution and predication is mistreated (cf VII.4).

"Frege was a formalist - of that there is no doubt. He be-
longed to the "higher" formalist school where rashness and
arbitrariness are abhorred, where not just anyone may de-
termine the rules.
Thus it is here that our mathematical logic comes into the
picture, and never before has logic been so pure, so free from
"foreign elements". Questions regarding the origin and deve-
lopment of concepts become irrelevant from the viewpint of
logic; modal, grammatical and linguistic aspects become irre-
levant from the viewpolnt of logic; (one recalls Frege's
bitter polemic in Begriffschrift and Grundlagen against
those who include such considerations in their Iogical ana-
lyses : epistemological considerations become irrelevant from
the viewpoint of logic and the same applies to ethical and
aesthetic considerations and questions concerning use, in-
terest, aim and intention. In brief, everything that gives
human concept formations their content and vaiue becomes
irrelevant from the viewpoint of logic! It has been possible
to free logical forms from all content, taking the new
mathematics as the model. It has been possible to formulate
the laws of "pure thought" by undermining the concept of con-

B) cf. also op.cit.:152-7. 
tent or meaning itself; by inventing purely logical content (Frege"s begriffliche Inhalt), a "formal content" which 10gical semantics (now also beginning to be set over against inguistic semantics) has later to take care of.

Modern logic, primarily through the discovery of formalization (in the arithmetical-algebraic sense of the word), constitutes the culmination of the separation of form and content, of the "purification" of theoretical thinking that began with Aristotle. One could perhaps say that Frege cuts the last tie - ordinary language. Ordinary language became ein Hindernis, as Frege puts it in his Begriffsschrift, in the search for the true language, the logical language (in the same way as ordinary reality has often also constituted an obstacle to philosophers in their search for true reality). The important thing here is not just the fact of the discovery of formalization; what is essentially new is the spirit that makes possible the acceptance of this discovery - the spirit according to which formalization becomes something respectable, legitimate and praiseworthy (consider, for example, the formalistic ideal for evidence for which Frege constantly agitates in his longer writings and contrast this with Kant's clearer conception of mathematical proofs - even If this too was a "pure" way of thinking).

(Stenlund 1980:156-7)

The thesis that logicians tend to think of the world as made up of static objects ties in with the observation that logic is poor at conceptually analyzing change. "A logic which is concerned with the conceptual scaffolding of a dynamic world is still largely a desideratum" (von wricht 1965). Ejerhed (1979) notes that this also holds for attempts at constructing tense logics. In $\operatorname{logic}$, change is consistently represented as the difference between states, rather than taken to be a primitive and basic notion in its own right. Yet, as Ejerhed notes, changes may well be more important than states in animal and human perception and cognition (cf, also Miller a JohnsonLaifd 1976). It may be added that change is clearly much more salient as a basic dimension in the production and understanding of face-to-face interaction than in (most aspects of) the communication by written texts.

9) Translation by Brian Beattie. 
In conciuding this section on logic and formal languages, let me call attention to a completely different point having to do with the written language bias in modern linguistics. Logicians have traditionally argued that natural language - being so vague, ambiguous, elliptical, "illogical" etc - impedes logical thinking (cf. the quotation above). This was, and presumably still is, the main reason for developing the formal languages of logic and mathematics as more exact and reliable vehicles for "logically correct" reasoning. These languages were developed on the basis of natural language in written form, and they are in fact used as very special, strictly regimented variants of written language (various forms of symbolic notation). There are entirely explicit rules for the outer form and the interpretation of these symbol languages. Now, it is important to notice that very influential scholars in modern linguistics have borrowed largely from logic and mathematics their conceptions of what a language is (or should be). We have witnessed, in the last two decaces, how linguists and philosophers have argued that natural languages are, after all, not that different from formal languages. In fact, the former should, according to some currently popular doctrines, be amenable to just as exact charaterizations as the latter. In our recent history, this argumentation has been carried through in two steps. First, Chomsky (e.g, 1957) argued that the syntax of any natural language should be given in terms of an axiomatized finite system of rules capable of enumerating all and only the grammatical sentences of the language (cf, V.4). Later, Montague (1974) proposed that the semantics of a natural language be given in terms of a formalized, intensional logic. Thus, he declared programatically: "I reject the contention that an important theoretical difference exists between formal and natural languages" (1974:188). As we all know, Chomsky's and Montague's views have had a tremendous impact on modern inguistics. In view of the fact that formal languages are so dependent on writing and written language, these currents in today's mainstream linguistics have increased the written language bias. 


\section{VII,9, Semantic representations}

A written sentence or text is static and atemporal. It is in principle simultaneously accessible in its entirety, although of course, its production and the single scannings in its perception are of course distributed in time and subject to time limitations on e.g. short term memory. The left-to-right organization of a written sentence is spatial rather than temporal. It is typical, however, that linguists, and similarly (but perhaps less consistently) experts in philosophy, psycholinguistics, artificial intelligence etc, regard the various "linguistic representations" (semantic, syntactic, phonological) of sentences and discourses as equally static and atemporal, no matter whether they are concerned with writing or speech. It seems to be tacitly assumed that what is true of a written sentence is also true of a spoken utterance, as if the distinction between sentences and non-sentence-like utterances were uncontroversial, and the transposition from one medium to the other unproblematic.

It is commonplace to argue that the surface-syntactic structure of a sentence or utterance exhibits both linear structure (leftto right organization) and hierarchical phrase structure. The latter point is, however, rather debatable; it has not been proved that surface structures must have connex tree structures with single roots, 10$)$ and this of course holds a fortiori for spoken utterances. As regards the left-to-right organization, it reflects the temporal structure of the corresponding stream of speech behavior, but this fact is not incompatible with the contention that the linguistic structure is atemporal, i.e. (at some point in time) simultaneously existing, and static. In fact, most theories of speech performance assume that in production the linguistic structure (of the whole clause or sentence)

10) cf. Hetzron 1973, Hudson 1980, Dahl 1980. 
is first constructed in its entirety and hence exists before it is executed in overt speech, and similarly this very same structure is assumed to be assembled at some point in time during the perception process.

It seems, however, that at least some linguists and psycholinguists are becoming increasingly inclined to admit that spatial left-to-right dimension of the written sentence has a temporal rather than a spatial counterpart in the spoken utterance, also as regards its linguistic organization. But this is usually applied only to (surface-) syntactic and phonological structure, not to semantic representations. The atemporality and staticness of the latter seems indisputable in the eyes of most people; after all, many linguists have maintained that the semantic representation of a sentence or utterance displays only hierarchical structure and no linear (left-to-right) organization. The question that now arises is: Is this really selfevident? Let me suggest a few reasons why it need not be that way.

First of all, if we are willing to assign to the outer form of an utterance a temporally distributed linguistic structure, then we should do the same as regards semantic structure. After all, semantic structure and outer form are strongly interrelated and are best considered two aspects of the same activity. Semantic structure may be thought of as consisting of various conditions or structure assignments on continuous mental activities, i.e. the stream of consciousness, the flow of interpreting activities accompanying either overt verbal behavior (utterances) or covert behavior, i.e. the kind of thought that is heavily dependent on verbal means ("thinking in words"). Thoughts and verbalizations arise, grow, get completed and disappear with time, and semantic structure and outer form are aspects of this temporal process (cf. VII.10). Apart from these admittedly abstract characterizations, we can, furthermore, note that there are many semantic aspects, traditionally discussed in semantic theory, which seem naturally amenable to a 
characterization in terms of dynamic and temporally distributed dimensions; some examples are functional sentence perspective (FSP), theme-rheme, background-focus etc.

Now, linguistic structure is of course something very abstract, and must not be equated with the behavioral processes themselves (VII.10). Therefore, it is a moot point whether this structure is temporal as such; perhaps it would be a category mistake to argue in that vein. What remains true, however, is that the processes which are subject to linguistic structure (and without which there would be no language), are temporally distributed, and this fact must be given due attention in our analysis. 11) In the production and comprehension of speech, the entire configurations of phonological, grammatical and semantic conditions corresponding to the "linguistic structure" of sentences and larger units (i.e, units of the kinds that linguists generally contemplate) are presumably not simultaneously attended to and/ or otherwise mentally available. Rather, they are subject to piecemeal processing.

The reader should note that so far I have only discussed the semantic representaitons of utterances, i.e. the verbal means used in communication processes. I will presently advert to the nature of the representations of knowledge, the memory models of discourse universes, that are built up, exploited, and altered in the communication process. Could knowledge structures be considered atemporal and static, even if we admit that utterances and thought processes are time-distributed and dymanic?

11) I am not convinced that the adoption of a procedural semantics instead of, say, semantic networks and dependency trees makes any difference in this regard. On procedural semantics, se e.g. Johnson-Laird (1977). 


\section{VII.10, Knowledge structures}

In the fields of semantics, artificial intelligence and cognitive science, an important theoretical issue concerns the ways in which speakers store and use the knowledge that they need in order to communicate, and to perceive and understand language and the world. Scholars have assumed the existence of different types of knowledge structures, some termed "models", some depicted as networks of propositional knowledge. Another distinction is that between procedural knowledge and declarative knowledge (Winograd 1975). However, it is far from clear what is actually implied with these distinctions. Johnson-Laird (1980) wrestles with several of the basic issues and concludes, contrary to some other commentators (e.g. Pylyshyn 1973, Anderson 1978), that there are indeed a few differences in the empirical implications of postulating mental models as opposed to (merely) propositional knowledge. While propositional knowledge is supposed to be language-like in character (discrete and digttal rather than continuous and analogical).

"a model represents a state of affairs and accordingly its structure is not arbitrary like that of a propositional representation, but plays a direct representational or analogical role. Its structure mirrors the relevant aspects of the corresponding state of affairs in the world".

(Johnson-Laird 1980:98)

Mental models are not pictures (such a view leads to the wellknown absurdities of assuming an infinite number of homunculi), but nevertheless they seem to be some kind of (abstract) images. They allow the construction of subjectively (phenomenologically) accessible images, which are, among other things, amenable to mental transformations such as rotations and expansions (cooper 1975). Kosslyn (1980) suggests that mental imagery draws on at least two codes, an analogical "surface image" code that arises from some kind of "deep structure" code (the nature of which is unknown) and a propositional code which contains information that can also be used in the process of generating mental images. 
Cognitive science is a vital field, which shows a lively discussion of theoretical and philosophical issues, and it is of course impossible to do fustice here even to the most important arguments. However, a few reflections may be called for in the light of our own major topics. One cannot help observing that most of the knowledge structures of the types postulated in artificial intelligence and cognitive science seem to display some fundamental properties of written or pictorial representation, cf. networks or graphs with nodes containing propositions, and analogical "mental models" respectively. This in turn suggests an interpretation according to which a knowledge structure is conceived of as a more or less fixed, static and context-free object, which is exploited in thinking and communication, where various parts of it would be retrieved, displayed, combined or otherwise manipulated, and finally perhaps transferred in communication (as if communication were a kind of transportation of ready-made pieces of information, cf. x.1).

The assumption of large-scale integrated coherent knowledge structures is thoroughly unrealistic. It seems unlikely that we have ready-made models of all (or most) of the various parts of the world that we are acquainted with. Our knowledge of any universe of discourse has to be fragmentary and flexible, so that it can be used in many ways and for different purposes. It seems impossible that any normal language user could have a stable model of, say, the government of the United States. The reason is that his knowledge is by necessity multi-faceted and can therefore be organized under many different aspects. One could, e.g., focus on the present U.S. government in relation to the previous ones or possible alternative administrations, or one could think of U.S. governments in general and their functions. This in turn could be looked upon from different angles; what status is allotted to the govermment by the constitution, and what are the actual roles played by the U.S. government in practice? The U.S. government may be seen in relation to foreign governments or to the various sectors and layers of the American society. One could compare the functions of the govermment as they are with what they should be, one could consider the staff as govermental officiers or as particular human beings etc. 
There is an infinite set of different aspects, under which we can analyze reality and organize our knowledge of it. Human beings encounter an infinity of situations in life, which are all different with respect to which facts are possibly relevant, which are actually relevant, and, among these, which are essential and which inessential (cf. Dreyfus 1972:168ff). In these situations people behave in ways which are usually orderly but not governed by well-defined rules. Nor can such intelligent behavior be explained by recourse to well-defined "models of the world", because any particular well-defined model, 1,e. a specific organization of knowledge of a given section of the world, would be too unadaptable and too dependent on the purposes for which it was originally set up.

The assunption of integrated stable knowledge structures does not square with the openness of the human mind. It seems to underrate strongly the agent's reorganizing activities in recall, association, verbalization and argumentative reasoning; the subject matter is exploited in a creative fashion and in constant interaction with the environment and the specific tasks to which the activities are oriented. No static model of the world could satisfy the needs of such an active subject. In fact, we could say with Dreyfus (1972:212) that human beings utilize not a model of the world but the world itself.

It remains true, of course, that humans make use of memorystored knowledge. It is also evident that there are various specific associations in this memory-stored knowledge, although the whole structure cannot be very stable and integrated. When we use our knowledge, only very limited parts can be attended to at one time. The stream of consciousness moves from fragment to fragment. Perhaps these fragments are what chafe (1979) called foci, which "are, in a sense, the basic units of memory" and "represent the amount of information to which a person can devote his central attention at any one time" (180):

"My guess at the moment is that people have in memory a large number of foci involving knowledge of particular objects, events, and so on plus a relatively small number of principles of coherence by these foci can be organized into the larger units that appear in language as sentences. These principles of coherence have to do with unity in terms of images, schemas, goals, and the like".

(Chafe 1979:176) 
It may be objected that it might be possible, after all, to build up a well-organized model in at least certain cases, where the tasks demand so, e.g. If one has to read a certain text, memorize it and then later relate it. But again we are probably not faced with a very stable structure, like a chart, a picture or a written text. Rather, our knowledge remains based on fragments ("foci"), but in addition we may have memorized certain tricks that enable us to go from one fragment to the other in a certain more or less fixed order. Thus, though our knowledge may consist only in local pointers (at a given place we know how to proceed to the adjacent fragment), we may still be able to follow consistently a specific path through the whole story. This path in memorizing and recall would then reflect the temporal organization of the discourse.

Obviously, we cannot here make any attempt at solving the problems of what kinds of knowledge structures must be posited in cognitive psychology and artificial intelligence. Such an endeavor is entirely beside our main point, which is merely to. suggest that some tendencies in current thinking on knowledge structures are biased by the traditional written language focus. We often think of knowledge in terms of (more or less) atomic facts organized in charts, diagrams, pictures, lists, tables, networks etc. Such structures can only be produced if we are allowed to use paper and pencil, or, on a much more sophisticated level, a computer. Computers represent a natural extension of the applications of written language. They use written language rather than spoken language, and to the extent that linguistic behavior has been simulated in artificial intelligence, it is not the dynamic social interaction in spoken dialogues that has been aimed at. ${ }^{11 a}$ )

11a)It is true that there are a few studies which attempt at dealing with "artificial intelligence and conversation" or with simulations of dialogue exchanges (e.g. Allen \& Perrault 1980, Perrault \& Allen 1980). However, apart from those studies of natural spoken discourse which have not led to program formulation or actual simulation land hence have nothing particular in common specifically with computer sciences) (e.g. Reichman 1978), it seems that this work is entirely based on the medium of written language. The only property which it shares with spoken discourse, is the interactive character, the relatively bidirectional flow of questions and answers between system and user. 
Computers work with language and knowledge on a digital basis. Now, it cannot be denied, of course, that all (human or nonhuman) kinds of cognitive processing of sensory inputs seem to involve such things as selection, quantization (perhaps digitalization), categorization, assimilation to schemas etc. Nor should we ignore the fact that our inability to observe directIy mental processes as such leaves us with the sole possibility of characterizing the processes in terms of their outcome (IV). But I would maintain that writing strongly reinforces these things and specializes them in certain directions. This has to do with important inherent limitations in the media. For example, the lack of a context must be compensated for in written texts and in computers. Humans constantly interact with their environment during spoken communication, but computers have to have an internal model of the environment with a very large, almost infinite amount of data ("knowledge") stored. Computers do not get anything comparable with normal sensory input. It would therefore be preposterous to assume that computers could simulate the human condition in a veridical way (cf. Dreyfus 1972).

With computers we can produce complex structures, pictures and lists, in which great amounts of richly structured elements can be integrated and systematized. The products are preserved over time and can be surveyed, manipulated, adjusted and improved, reorganized in part-wholes etc, in principle without limits and without the interaction of oblivion and the limitations on normal human attention. Are there any interesting analogies between this and the knowledge structures set up and used in oral communication, where the linguistic products are fugitive, the spans of attention and short term memory limited, and the flow of incoming signals and impressions of the social interaction virtually endless? The "knowledge" involved in the social interaction of spoken dialogues is to a large extent practical knowhow, skills of various kinds, and this crucially involves emotive and social (phatic) aspects, i.e. not only cognitive aspects such as intellectual knowledge of atomic facts. Is this at all amenable to simulation on digital computers? 
In concluding this section, it would be fair to point out that the attitudes to knowledge that we have attributed to artificial intelligence and to the computer sciences in general, have a much wider societal basis than we have so far assumed. It seems to me that there is in our Western culture a ubiquitous tendency to conceive of "knowledge" as something which has only objective referential aspects; we tend to look upon knowledge as facts, as fixed and stable pieces of information such as can be found in text-books, waiting there to be acquired and used, knowledge as something which is already complete and stored in archives and repositories. Such a conception of knowledge is very narrow. It leads to difficulty in explaining our abilities to combine basic elements in new ways and to apply them to new problems, and it does not take into account all the emotive, practical and social ${ }^{12)}$ aspects of our social interaction and, in general, in our ways of dealing with our environment in a flexible, intelligent manner. Whatever the merits and limitations of this conception of knowledge are, it reflects the profound impact that the written language bias has had on our entire culture.

12) On these "functions", see $\S I X .2$. 


\title{
VII.11 Semantic representations and thought processes
}

\begin{abstract}
"All the major figures responsible for the classical phrasing of linguistic relativity, Herder, Humboldt, Weisgerber, Trier, Cassirer, Sapir, and Whorf, erred in precisely the same way. Each, under the sway of the Western inclination to objectify processes - an inclination characteristic of peoples who embrace alphabetic writing - has distinguished grammar (langue) from speaking. Each has hypostatized a socially shared grammar and has assumed that grammar to be an object which each individual reproduces in his mind. Each in different words and in different ways has claimed that mental life is identical to that grammatical object. The error of linguistic relativity does not lie so much in the claim that mental life has no independent existence outside of language, but that mental life is identical with language as an object rather than with language as a process".
\end{abstract}

(Washäbaugh 1980:210)

For various reasons, one of them being the long-time insistence that linguistics must be an independent discipline with its own object of study (V.I), linguists and psycholinguists have been prone to postulate "mental objects" and "mental processes" that are claimed to be specifically "linguistic" in nature. Such an assumption appears to distort reality at several levels. For example, what a speaker produces when he constructs and executes an utterance is not a mental "linguistic" object (e.g. a plan) but a stream of phonetic behavior ("vocalizations") such that it meets certain conditions defined by its expression plan ("outer form", cf. Iinell 1980). When a listener perceives, say, a word, he does not perceive a linguistic (phonological or whatever) form, e.g. a string of phonemes, but he perceives a section of someone's phonetic behavior as a word, i.e. as a stretch of behavior that is conventionally associated with a set of semantic, grammatical and phonological conditions. Similarly, when someone understands an utterance, he does not construct a semantic representation for the linguistic expression involved, but he uses semantics as clues in the search for an interpretation in the environment, in his inner world or in both (cf. VII.3-5). 
Furthermore, it seems to me that many versions of information processing psychology, artificlal intelligence etc easily leed to the hypothesis that mental operations are carried out in "the language of semantic representations" (or deep structures, transformations), that thinking consists in the application of semantic procedures etc. For example, this view is articulated in Fodor's "The language of thought" (1976). 13) such a view may easily force us to accept a strong version of the Sapir/ Whorf-hypothesis, something which must be regarded as a rather unfortunate consequence. It is of course impossible to discuss the enormous problem of the relations between language and thought here, but it may be remarked that our traditional theory of linguistic rules as conditions on behavior seems to make a more natural and plausible alternative available. Rules are not processes but conditions on communicative behavior and other kinds of behavior such as perceptual exploration, imagery, problem-solving etc (cf, VIII,4). Thinking is undoubtedly a form of very language-dependent (internal)behavior, and as such it may follow, or be subject to, semantic rules of various kinds, but it does not consist in the manipulation of purely "linguistic" semantic representations. The latter view would turn language into a completely self-contained system without any basic relation to the physical and social world.

13) For critical discussion, see 0lsen 1979, Fortescue 1979, as well as the now classical critique of cognitive simulation and artificial intelligence in Dreyfus 1972 . See also Winograd's (1980) reorientation.

14) Thus, e.g... a sentence is an abstract entity consisting of a configuration of grammatical conditions (that may or may not be met by specific stretches of speech or written language (VI. 1). Phonological structure is seen as phonetic conditions on the stream of vocalizations (VIII.4). SimilarIy, in $\S$ VII.9 semantics is described as conditions on mental activities. 
VIII, PHONOLOGY

'There is, incidentally, nothing particularly surprising about the fact that conventional orthography is, as these examples suggest, a near optimal system for the lexical representation of English words. The fundamental principle of orthography is that phonetic variation is not indicated where it is predictable by general rule'

(Chomsky \& Halle 1968:49)

\section{VIII.1. Phonological structure}

Phonetic behavior and the resulting acoustic signals are continuous dynamic phenomena. The various phonetic gestures involved in speech production overlap and have no abrupt onsets and offsets. Yet it is generally assumed that phonological structures (phonological forms, phonological representations) underlying speech consist of linear sequences of discrete, static segments. This implies that phonological structures would be structurally similar to strings of alphabetic letters. Such letters are in fact used as phonological notation.

The use of alphabetic writing as the metalanguage of phonology is something which may be assumed to have a significant impact on our theories of phonological structure. Before going on to a discussion of this matter, we should notice, however, that not only the abstract, underlying phonological structure of words is notated by means of discrete graphic symbols. We also use more "concrete" representations of the pronunciations of words and utterances, so-called (narrow) phonetic representations, which are likewise couched in a (modified) letter notation. This means, in all probability, that our view regaraing phonetic structure is also influenced by the outer form of this written metalanguage. In any case, it would be utterly naive to believe that phonetic transcriptions, no matter how "narrow" they are, are some kind of mechanically computable, "objective" representations (or reflections) of the phonetic signals. On the contrary, they are the result of a conventional 
transformation of speech into writing, and we need have access to implicit (conventional) rules in order to be able to convert them "back" into speech. It seems probable, says Ladefoged (1967:52), "that our lack of knowledge of what we are doing when we make phonetic transcriptions is actually hampering our own work as descriptive linguists".

The phonemic principle and the idea of the double articulation of spoken language are probably historically dependent on the existence of alphabetic writing. Thus, if the continuous and varying stream of behavior has to be notated in writing, there arises a need for an economic set of discrete signs, e.g. letters or other symbols (such as pictures). Therefore, a practical notational system presupposes an analysis in terms of segments of some sort. The next step in the argumentation implies that these segmental units are not only workable units of analysis, they are in fact inherent properties of the subject matter; hence phonologists discovered that there were in fact segments underlying overt behavior.

It would be stupid to deny that the idea of underlying segments has some kind of basis in speech production and perception. First of all, the drive towards categorization applies to the perception of speech as well. For this and other reasons patterns and routines are developed also in speech production; the same motor elements tend to be used in the articulation of all words in the language. There is substantial evidence for units like syllables, syllabic constituents (onsets, nuclei, offsets), vowels and consonants as units of production; common slips of the tongue (such as) spictly streaking for strictly speaking, strunction and fucture for structure and function, lawn drawn for line drawn are but one type of evidence. Nevertheless, it is no doubt true that writing and the ability to read and write enhance our experience of speech as being composed of segments. What is at stake here is not the general idea that vowels and consonants are components of speech, but rather the much stronger hypothesis inherent in most phonological theories, i.e. that the phonological structure of a word is just a linear sequence of nonoverlapping segments. Fowler formulates the basic point of this 
"strong segment theory" like this:

"Segments in a planned sequence are discreze in the sense that (abstractly stated), their boundaries are straight lines perpendicular to the time axis, so that the terminus of one segment is the beginning of the next segment".

(Fowler 1980:116)

According to mainstream phonological theory, each segment is a bundle of simultaneous features. Such a segment sequence is a basically spatial (rather than temporal) organization of thinglike phonological units arranged in a before-after sequence analogous to the left-right sequences of conventional orthography and conventional phonetic notation. In an extreme version, this theory excludes the possibility that suprasegmental features and syllable structure are phonologically significant.

If the phonologist's view of phonetic structure is influenced by the perspective formed by alphabetic writing, this is true of the layman's thinking about speech to an even greater extent. Aside from the fact that sounds (phonemes) and letters are hopelessly mixed up in the linguistic thinking of most laymen, it is clear that writing distorts our phonetic intuition and make us deaf to certain phonetic realities, notably those which have

1) Note that some analysts want to break down contour tones and intonational contours into sequences of static level tones (cf. Woo 1969, Hill 1958, Pike 1945). In general, there is a tendency among phonologists to prefer segment-based solutions. In segmental phonolgy one may perhaps also discern a slight bias towards analyses which assign distinctivity to consonants rather than vowels; thus, "secondary" characteristics of vowels such as nasalization, palatalization, and pharyngealization, are often derived from underlying consonantal contrasts rather than treated as prosodic features of sequences of syllables or vowels. (An extreme example is provided by Kuipers (1962) who ascribes no vowel contrasts at all to the Kabardian language). One should then note that alphabetic symbol systems are usually more powerful in representing consonants than vowels. Semitic writing systems are an obvious example. 
no counterpart in common orthography. For example, phonetically untrained listeners do not hear the difference in aspiration between the p:s of peak vs speak, nor do they hear the vowel murmur after the stop in a stop-liquid initial cluster in e.g. please and prayed (which makes them almost homophonous in certain speaking styles with police and parade). Furthermore, the relative inability to perceive prosodic phenomena is another case in point. One may also mention the wide-spread belief that words are regularly separated by silent intervals (pauses) in speech, in analogy with the empty spaces of written texts; actually such pauses are relatively infrequent, which can be seen at any registration of the acoustic signal.

Literacy may conceivably have effects on speakers implicit understanding of other aspects of phonology too, e.g. morphophonology, 2) There is some evidence from various psycholinguistic experiments (recall of nonsense forms, cf. Myerson 1975) that subjects who can read and write English well and thus are sufficiently acquainted with English orthography tend to associate pairwise such heterogeneous vowels as English [ail]-[I], $[\hat{f} I-[\mathrm{e}],[\mathrm{e}]-[\mathrm{a}]$ etc. Young children, on the other hand, seem to base similarity judgments on phonetic properties (as demonstrated in spontaneously invented spellings, Read 1971). To the extent that these results hold true in general, the reason for adult speakers' associations is of course the fact that single vowel letters are used in English orthography to designate pairwise quite different vowels, i.e. $\underline{i}$ for [âl] and [I], a for [a]] and [ae] etc. 31

2) Morphophonology is best treated as part of morphology rather than phonology (Linell 1979a), but this is something which we can ignore in this context.

3) The same orthographic principles are applied even in the quasiphonetic transcription of many common English dictionaries, where e.g. the pronunciations [ai, $\left.\varepsilon_{1}\right]$ etc are given as $\langle I, \bar{a}\rangle$ etc. 
Let us now return to our main theme, the contention that the idea of double articulation derives some of its motivation from alphabetic writing. It seems to me that this hypothesis receives a certain amount of confirmation from the recent history of the theory of the sign languages of the deaf. Earlier, many people in the field seem to have argued that signed languages are not doubly articulated (Healy 1973). However, later on some scholars (e.g. Stokoe 1960, 1972) "discovered" that they did in fact display double articulation. This discovery seems to have been connected with the invention of discrete written symbols to represent the movement components of gestures (cheremes). ${ }^{4}$ ) When the medium of representation required an analysis in terms of such distinctive features, the theory that these features do in fact exist as inherent parts of the subject matter was immediately naturally at hand.

Some of the insights of phonological theory, e.g. as regards the capacity for meaning differentiation inherent in different sound gestalts, has been implicit in linguistic thinking for centuries, even milennia. In fact, the development of alphabetic writing presupposed some kind of phonological analysis of speech. Also, the practical goals, for which phonology has proved necessary, are largely connected with the need for devising alphabets for new languages. This shows up clearly in the practice of American descriptivists following Boas, Sapir and Bloomfield; Pike gave his book 'Phonemics' (1947) the subtitle 'A technique for reducing languages to writing'. It may well be that even contemporary phonological theory is more suitable for investigating optimal orthographies than for discovering the structure of spoken language.

4) Cf. also Birdwhistell's (1970) analysis of gestures and bodiIy movements in general in normal hearing subjects. Birdwhistell tries to isolate elements of behavior, called kinemes, in analogy with phonemes. 


\section{VIII.2 L-image acoustique}

So far I have identified only one of the ways in which the written language bias has entered phonology, i.e. through alphabetic notations used for phonetic transcription. However, there is at least one more avenue by which the written medium has influenced phonological thinking, viz. through the theories of perception.

Given that phonological structure is ascribed some kind of psychological vallaity, it is reasonable to argue that the phonological structure of words is postulated or constructed by language users on the basis of the sensory information available about acoustic signals. The phonological form of, say, a word is seen as a representation of the phonetic signal, as a structure which is perceptually determined. Instead of representation, other terms have been used, e.g. sound image, mental imaginary counterpart of a sound (Lautvorstellung), or in Saussure's words image acoustique.

Behind these notions lurks an old theory of speech perception. This theory was in its turn modelled on traditional theories of visual perception, according to which we form mental images, inner pictures, of what we see around us. In short, the theory implies that we do not see the outer reality "directly" but we see a picture of it. One of the arguments for such a theory has been that we can see the same stimulus configuration in different ways; we form, as it were, different pictures of it. (After a11, the eye does form retinal images). Such theories are fraught with difficulties; among other things, they lead to infinite regressions (who is it that looks at the inner image?). In today's thinking about perception they are being abandoned in favor of theories of "direct" perception, according to which the perceiving agent actively searches for and reinterprets patterns into stimulus configurations (Neisser 1976). 
What is important, however, is that the theories of visual perception involving mental images were clearly inspired by the existence of real images and pictures, i.e. static thing-like representations on stone, wood, leather or paper. Such pictures may be more or less close to what they represent; at any rate, they may depict the same fragment of reality in different ways. It therefore seemed quite natural to explain our ability to perceive reality in different ways, under different aspects, by assuming that we form different mental images of it. This type of theory was later transferred to the area of auditive perception as well. The same phonetic behavior may be perceived in different ways, e.g. by speakers of different languages, and this could be explained by assuming the existence of different ways of forming mental images, i.e. phonological representations, of the phonetic behavior involved. Again, we see how our picture (sic!) of reality is dependent on our experience of written texts and pictorial representations.

It might be added here that a careful analysis of the concept of phonological form shows that an interpretation in terms of sound images is unacceptable on several grounds. This in no way amounts to denying that our knowledge of the phonological structure of speech is perceptual in origin. However, we need not end up with a theory that implies that we hear percepts, or phonological forms, rather than the actual phonetic signals themselves: we do hear phonetic events, but we hear them as something particular, under certain aspects. The most important aspect implies that the phonetic signals are heard as instances of meaningful communicative behavior in a certain natural language (Linell 1982a). 


\section{VIII,3 Phonological rules}

It took linguists a very long time to admit unequivocally that letters and sounds (phonemes) were two quite distinct things. However, one may still ask the question whether the ties between the two have been definitely cut off or not. Modern phonology thinks of phonological representations as referring to mental things, i.e. mental counterparts of sounds, auditive images or the like, which are assumed to exist "prior to" the phonetic behavior (VIII.2). Phonological rules, which, by the way, are often assumed to be isomorphic to putative performance processes (VIII,4), are depicted as substitution processes applied to these mental objects. In the allophonic rule of (1), one thing ([g]) $\left\{\begin{array}{l}\mathrm{m} \\ \mathrm{n}\end{array}\right\} \longrightarrow \mathrm{g} / \longrightarrow \mathrm{f}$ is substituted for another $(/ \mathrm{m} /$ or $/ \mathrm{n} /)$, just as one symbol on paper $(\langle m\rangle)$ is replaced by another $(\langle\eta\rangle\rangle)$. (cf. Bailey 1979). (Such a formulation in terms of discrete replacements is particularly inadequate as regards "concrete" rules of hypo- and hyperarticulation, where the variations in pronunciation are gradual rather than absolute (Iinell 1979a:188ff)). In general, one may say that phonologists abstract totally from the time-distributed nature of the phonetic signal and perform their analysis of phonological structure as if they were indeed concerned with nothing but strings of graphic symbols. Once the phonetic data have been transcribed, they have taken on all the characteristics of the metalinguistic notation; they have been transformed in the minds of the linguists.

One cannot help noting that the concept of phonological rule must be given a rather odd interpretation in (orthodox) generative phonology (e.g. Chomsky \& Halle 1968, Postal 1968). Accordingto traditional phonology, the most reasonable interpretation of the notion "phonological (or phonetic) rule" would be something like "rule for the language-specific exploitation of certain phonetic mechanisms"; thus, a phonological rule covers a regularity in the phonetic behavior pertaining to a specific language or dialect. Such rules are related to habits of behavior, which are partly subject to implicit norms of behavior. These rules are 
socially given and socially shared, which means that they are also socially acquired; the individual speaker learns the rules by inspecting and analyzing other people's behavior. However, in modern generative phonology we are faced with something entirely different. There, phonological rules are part of large systems of opaque ${ }^{5}$ ) rules operating on vary abstract underlying forms according to extrinsic ordering restrictions (see Chomsky \& Halle 1968, and for critical discussion Linell 1979a). Although the outputs of the whole derivations of such a system may be seen as conditions or rules for phonetic behavior, neither the underlying forms nor the specific generative rules as such may be construed as norms of phonetic behavior. While linguistic rules in the traditional sense must be learnable (cf. above), it seems inconceivable that a generative phonological rule system could be acquired by social learning. This point has been raised as a fundamental critique of the whole theory (e.g. by Derwing 1973), but Chomsky draws an entirely different conclusion; he assumes the whole system to be partly derived from very specific innate structures of the human mind (or brain). Furthermore, rules are interpreted as mental (and presumably causally efficient) processes, which again is something completely different from norms of behavior. (This view ${ }^{6)}$ is implicit in most of generative psycholinguistics, but it is most clearly spelled out in Katz 1964).

5) An opaque rule has a range of application which cannot be abstracted from a mere inspection of surface phonetic strings. For a mere technical discussion, see Kiparsky 1973.

6) The same kind of reasoning (to be applied here) is also applicable to other types of generative-linguistic rules, e.g. transformations in syntax. 
However, upon closer consideration it turns out that generativephonological rules may in fact be assigned an interpretation which is by and large "rules or norms for behavior". But then we are no longer concerned with the original object of study, i.e., phonetic behavior in speech communication. Instead, we are dealing with meta-rules in the sense of "norms for the linguist's analysis". Note that Chomsky derived his notion of rule from mathematics; a mathematical rule essentially states the conditions under which a given string of symbols may be rewritten as another string of symbols. This is also true of generative-inguistic rules. In fact, a phonological rule amounts precisely to a norm for the phonologist's behavior as he is practising linguistic analysis; thus, rule (1) says that under certain conditions strings like/mf/ or /nf/ may be substituted for, or rewritten (by the phonologist), as $/ \mathrm{mf} /$, or the other way around, under certain conditions / $\mathrm{mf} /$ may be analyzed (by the phonologist) as one of the underlying strings $/ \mathrm{mf} /$ or $/ \mathrm{nf} /$. Here we see how the whole theory is dependent on the written metalanguage; generative phonological rules state norms for how the (orthodox) phonologist may translate a given string of written symbols into another such string within the framework of a rewriting system that generates phonetic transcriptions, which in turn are devices by which the structure of phonetic behavior has been converted into writing. 


\section{VIII.4 The relation between phonoiogy and pnonetrcs}

Current theories of sound structure assume that there are two different, purportediy real modes of existence of speech sounds, i.e. the phonological strings of discrete, static and contextfree segments, and the continuous, dynamic, phonetic behavior. (Note, however, that also the latter phonetic behavior is conventionally transcribed in discrete letter notation (VIII.1)). This necessarily generates a number of problems for the theories of speech production and perception; How should these two seemingly contrary conceptions be reconciled? What role do phonological forms play in speech performance? These issues have been extensively dealt with elsewhere, e.g. in Linell (1982a), and I will therefore confine myself to a few brief statements here.

According to the standard view the phonetic processes of speech production are essentially coarticulatory. This means, in short, that strings of discrete, static segments (conceived of as some kind of mentally real things) are coarticulated in such a way that a dynamically varying continuum arises (e.g. Daniloff \& Hammarberg 1973, Hammarberg 1976, Kent \& Minifie 1977). The phonetic manifestation precess is characterized as "the assignment of phonetic effects to phonological causes" (Hammarberg:356), or as a "conversion" or "translation" of phonological features to "articulatory transitions" (Kent \& Minifie:131) 7). As Fowler et al (1980) have pointed out, this means that speech production implies a destruction of the clear-cut phonological structure, which turns into "an impoverished acoustic signal". Conversely, the process of speech perception involves the reconstruction of the phonological form on the basis of a pur-

7) "Translation theories" of speech communication will be discussed in $\$$ IX.I 
portedly "defective" signal. It seems to me that well-known values are reflected in such statements. The spoken signal is defective and messy, while the underlying phonological structure is as neat as a string of graphic symbols:

This type of theory with its conception of the relationship between "mental" phonology and "physiological-physical" phonetics creates a number of pseudo-problems, and it refuses to take phonetic behavior at face value, as something which is in fact orderly and structured, although not structured in precisely the way that the "strong segment theory" (cf. VIII.1) predicts. There appears to be evidence, from speech errors and elsewhere, that vowels and consonants, syllables and syllabic constituents, words and parts of words (stems, affixes) are, or may be, phonetic units. Furthermore, we can observe various prosodic phenomena having a suprasegmental and holistic character (cf. V.4). These properties are largely language-specific, and may therefore aptly be thought of as "the phonological structure" of the language involved. But this does not force us to imagine phonological units as some kind of "mental things" which get converted into behavior. That would entail a very problematic "translation theory" of speech communication (IX.1).

Rather, we should prefer another metaphor. Phonetic behavior is a stream of continuous vocal behavior, and the phonological structure may be seen as conditions on, or modulations, on a carrier wave of vocalizations (see Linell 1982a). The brain (or, if you will, the mind) is constantly active, and the perception of a certain stimulus pattern as a particular linguistic utterance (with specific phonological, grammatical and semantic properties) would involve inducing a certain change (increase and modification) of the patterns of activity, Such modulations would presumably result from a complex interplay between input stimuli and internally generated constraints (expectations, structural 
guesses). ${ }^{8)}$ In the latter phenomenon we can identify the role of linguistic structure:

"In the processing of speech, the function of lexical, syntactic, and phonological structures is that of constraints: they reduce the number of potential guesses available for the identification of the acoustic speech input."

(Sajavaara 1980:9)

8) In today's jargon: the process is both data-driven and conceptualiy driven. 


\section{IX.1. Communication as transportation of messages}

We have seen that linguists like to think of linguistic items as having fixed and stable properties, among other things inert, literal meanings. This way of looking at linguistic phenomena as objects is coupled with a very popular model of linguistic comunication, i.e. the one which portrays the communication process as the conveyance of a message, or, in other words, some kind of transportation of a certain, fixed message from the speaker to the listener, a transfer of given thoughts and feelings as if these thoughts and feelings were independent of and prior to the "encoding" and "decoding" processes in communication.

This view is associated with the metaphor that meanings are objects (VII.1):

\footnotetext{
"Meanings are objects.

Iinguistic expressions are objects.

Iinguistic expressions have meanings (in them).

In communication, a speaker sends a fixed meaning to a hearer via the linguistic expression associated with that meaning.

On this account it is possible to objectively say what you mean, and communication failures are matters of subjective errors: since the meanings are objectively right there in the words, either you didn't use the right words to say what you meant or you were misunderstood."
}

(Lakoff \& Johnson 1980:206)

The view that communication is basically a transfer or transportation of fixed messages shows up in many disguises, under several different but related metaphors. One such metaphor is involved in "the bucket theory" of meaning: ")

1) Osgood derives this metaphor from Malinowski (1949). Of course, Malinowski strongly opposed the theory of meaning underlying it. 


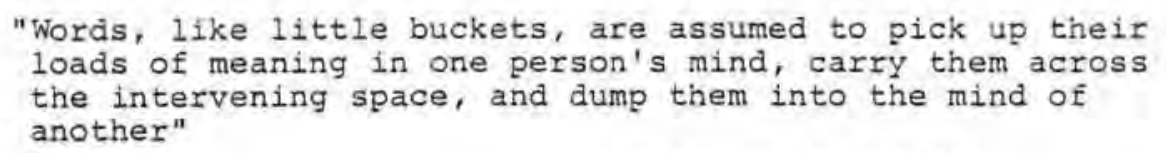

(Osgood $1979: 213$ )

Common to this and many other theories of linguistic communication is the view that words, sentences, and other expressions are containers loaded with meaning (content); words are, in other words, meaning-full (sic!). Reday, in an interesting paper (1979), treats the matter under the name of the conduit metaphor, a frame of thinking which implies that:

"(1) language functions like a condult, transferring
thoughts bodily from one person to another; (2) in
writing and speaking, people insert their thoughts
and feelings in the words; (3) words accomplish the
transfer by containing the thoughts or feelings and
conveying them to others; and (4) in listening or
reading, people extract the thoughts and feelings
once again from the words."

(Reddy $1979: 290$ )

Reddy points out that the conduit metaphor underlies many common English ways of talking about linguistic communication; it is deeply rooted in our (Standard Average European) culture and hence very difficult to free oneself from. Here are a few expressions that show how we have conventionally and inadvertentIy become accustomed to the conduit metaphor in our everyday language: ${ }^{2)}$

(1) Try to get your thoughts across better.

(2) Whenever you have a good idea practice capturing it in words,

(3) Try to pack more thoughts into fewer words,

(4) The lines may thyme, but they are empty of both meaning and feeling.

2) See Reddy (op.cit.) for many more examples. 
(5) Can you actually extract coherent ideas from that prose?

(6) He writes sentences in such a way as to seal up the meaning in them.

(7) His words carry little in the way of recognizable meaning.

(8) Please pay attention to what's there in the words.

Another metaphor for basically the same perspective on linguistic comunication is one which is particularly familiar to linguists, psycholinguists and communication theorists; it construes the communication process as a series of recodings, or mechanical translations, of the same message with a certain fixed meaning. Accordingly, this has been termed the translation theory of speech communication (Garrett 1975, Linell 1982a). In short, the theory says this. For some reason, a speaker comes up with a certain "idea" or "thought" which he wants to communicate to someone else. This "message" is therefore encoded in the speaker's brain into, say, patterns of neural activity which travel along nerve paths down to the various speech organs, where the message is translated or recoded into articulatory movements, which in turn give rise to a new form of representation, i.e. acoustic sound waves. These waves convey the message to the hearing organs of a listener, and there it is translated again into new codes. After a number of additional recodings, the message is finally regained in approximately its original form, when it reaches the listener's brain or mind.

The theory just sketched is obviously inspired by Shannon and weaver's classical model of technical information transfer. It recurs in almost every introductory textbook on linguistics or speech communication (e.g. Denes \& Pinson 1963). I have given It couched in basically physical terms, but essentially the same type of translation-theoretical approach permeates generative psycholinguistics, where, instead, scholars prefer to talk about abstract mental representations corresponding to the constructs of linguistic competence theory. Thus, the message is there automatically processed through a series of purportedly 
"linguistically significant" representations. For example, the production processes may be explained as a linear sequence of translations of the same utterance via representations such as the following:

(e.g.)
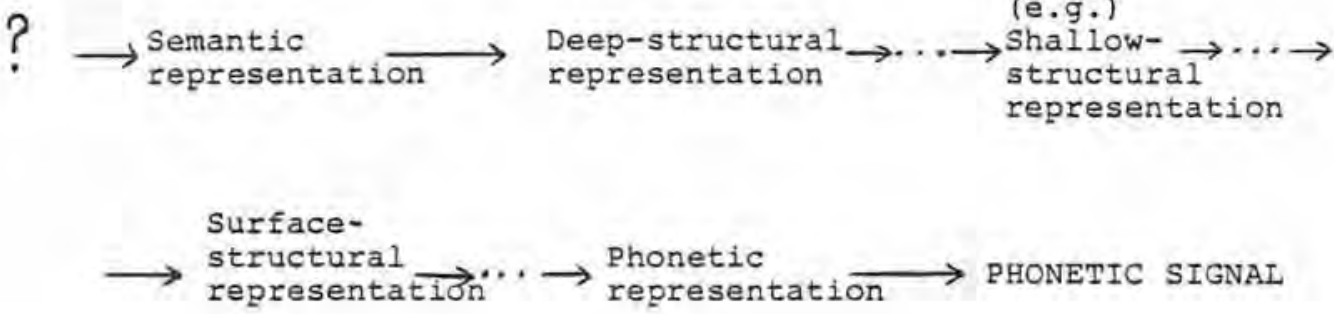

Exactly what "stages" are included in this series of translations naturally depends on the particular theory espoused. But the general outline is the same; although early ("remote") representations may be very different from late ("superficial") representations, they still "represent" the same message unit (usually a sentence, §VI.1) with the same fixed meaning. The process of perception and comprehension would, at least according to the most naIve theories, be essentially a translation in the reverse direction.

Proponents of such models of speech communication naturally admit that the whole process may be disturbed by "noise" in several ways. But the idealized version of the model clearly implies that one and the same fixed message gets across without distortion. If we assume that this model is reasonably adequate, then it follows that we should concentrate our efforts of linguistic analysis on studying the message at that particular stage, where it is most easily accessed, i.e. in the observable products (the phonetic behavior). It is thus assumed that the meaning of what is said can be gained simply by applying a linguistically correct analysis to these linguistic products. This is the view of the autonomous linguistic message once again. 
Two examples of translation-theoretical models in psycholinguistics and phonetics.

\section{THE SPEECH CHAIN}
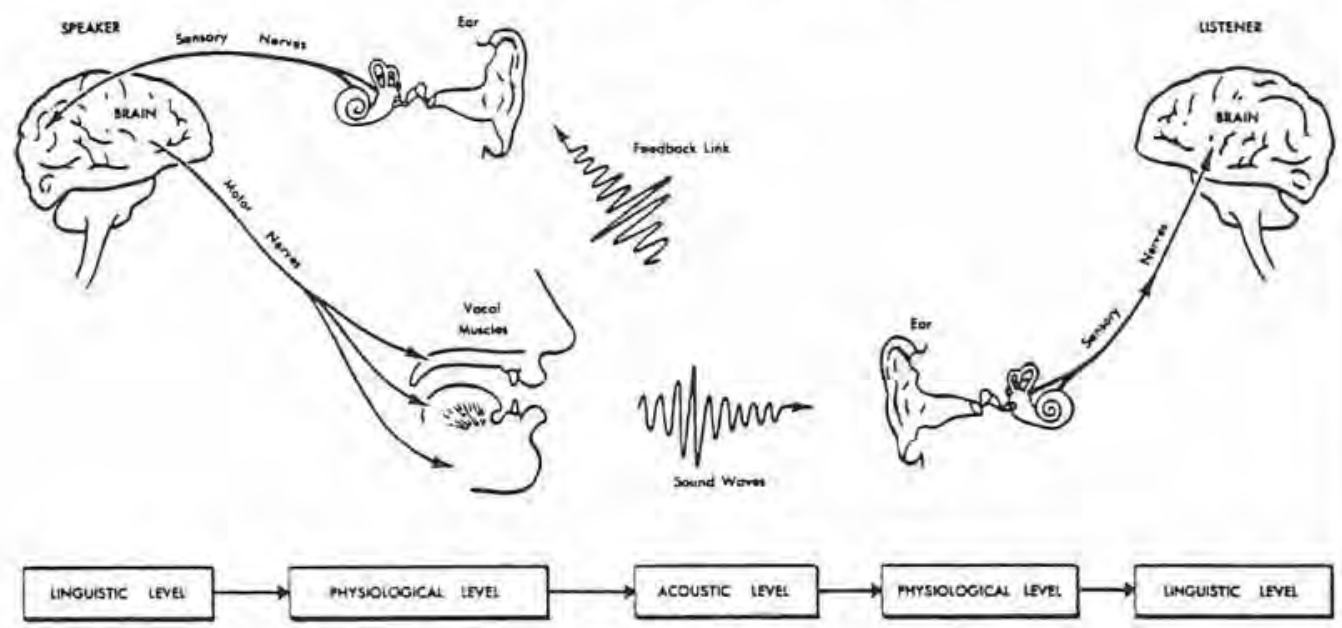

Fig. 1.I The Speech Chain: the defferent forms in which a spoien messogr exust in its progress from the mind af the speuker to the mind of the listener.

(from Denes i Pinson 1963:4)

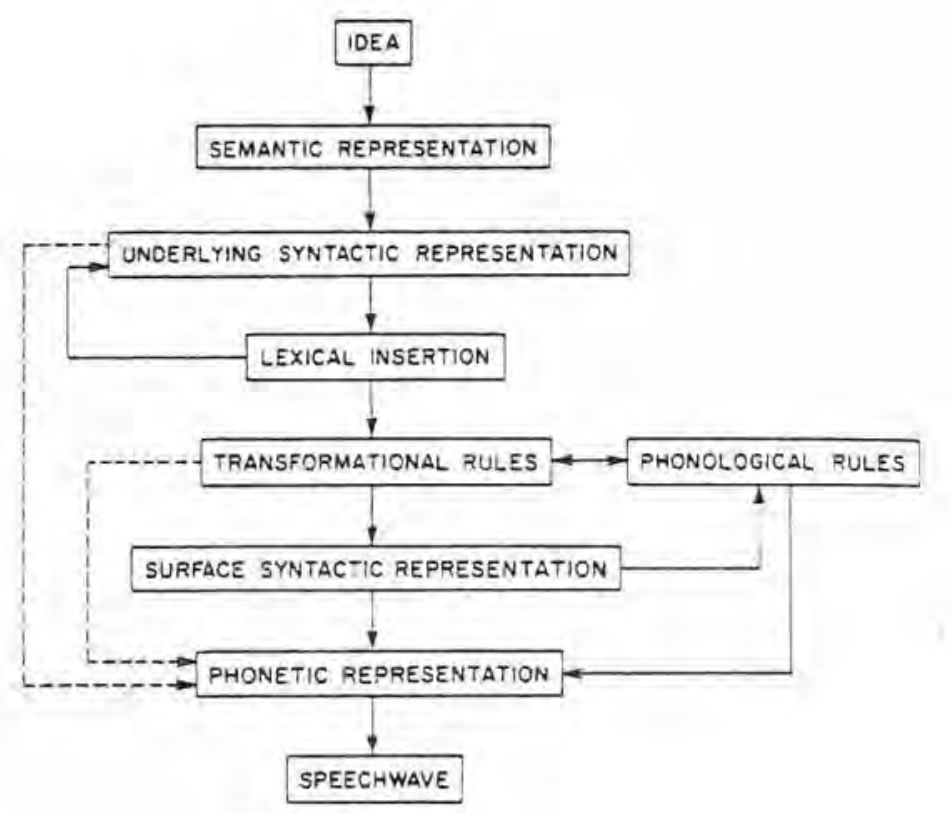

"A possible information-flon model of speech production"

(from Cooper 1980:298) 
The translation model is a thoroughly misleading and inadequate model as applied to speech communication in face-to-face interaction. It grossly underestimates the complexity of the social interplay between speaker and listener, and of their interaction with the surrounding situation:

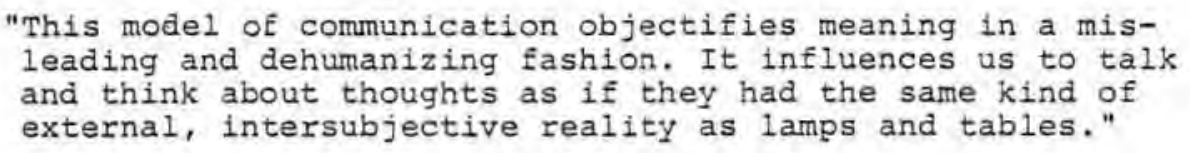

(Reddy 1979:308)

Translation models are inadequate for all sorts of meaningful communication between human beings; contrary to what these models suggest, we always take into consideration the productive and interpretive activities of senders and receivers ${ }^{31}$ situated in social contexts. However, it seems that the conduit or translation metahpors are less inaccurate as applied to communication by written messages. Indeed, it is obvious that the whole frame of thinking is inspired by such communication; someone writes a letter or a book, sends it away to an addressee/receiver for a group of receivers), who will read $1 t$, The written text has to be relatively explicit and relatively autonomous in various ways. Although the reader too must rely on yarious expectations and background knowledge, he is normally strongly guided by the text in his interpretive activities. Thus, the whole process reminds us to some extent of a mechanical transfer or transportation of a message. ${ }^{41}$

The reasons why this model cannot be exploited in the explanation of spoken dialogues are many. Most of them have already been discussed. Let me sum up a few points.

3) Note that the terms "sender" and "receiver" themselves belong to the framework of the conduit metaphor.

4) The same holds for communication between man and computer, which should be seen as an extension of communication by written language. 
First, the situational interpreations relevant to speaker and listener are never equal to the linguistic meaning associated with the utterance itself; any interpretation goes beyond the linguistic meaning which in itself is vague and allusive (VII.1,5).

Secondly, there is no complete linguistic meaning nor any fully developed intended interpretation in the mind of the speaker before the utterance has been compiled and its outer form has been determined; many aspects of meaning are the result of the verbalization process itself (Itnell, forthc,), and some interpretations are clearly discovered only after the verbalization (VII.5).

Thirdy, there are no uniquely correct situational interpretations; both speaker and listener may, e.g. vary in their depths of intention and understanding (VII.5). Any interpretation is in principle inherentiy negotiable and extendable, i.e. there are no fixed meanings being transferred in communication.

\section{IX.2. The functions of language}

Linguists tend to have rather definite preferences for certain theories of linguistic meaning and communication, as we have seen. Attached to these theories are certain implicit evaluations of which communicative functions are most important and most characteristic of language.

In this broad overview we may distinguish between functions along three different dimensions. First of all, one should single out the dialogic functions in social interaction from the monologic functions in thinking and other kinds of intraindividual comunication. This point will be further discussed in IX.3-4. It may be recalled that the conditions on written communication are such that both communicating parties may be said to use language in a monologic fashion. 
factors of the communication process and discern the following four aspects:

a) focussing on the sender: the expressive functions. What is communicated is, under this aspect, seen as expressions of the sender's beliefs, views, feelings, attitudes, volitions, needs etc. Naturally, some of these are unconscious to the sender and thus not intended by him.

b) focussing on the receiver: the evocative functions. Communication is here seen as directed towards evoking certain reactions on the receiver's part. What is conveyed serves to make him perceive or understand something, have certain feelings and attitudes, or perform a certain action.

c) focussing on the subject matter, 1.e. on the imaginary or objective reality that the message refers to: the referential functions. We are then concerned with how communication is used to refer and describe, to analyze, argue about, and explain things in the world.

d) focussing on the relation between sender and receiver: the social functions. From this point of view communication serves to establish and maintain social contact between the communicating parties. A great deal of oral discourse takes place simply because social situations and conventions require it, perhaps because one feels obliged to avoid an embarrassing silence. Some speak of the phatic function of language (Malinowski 1949:315).

Thirdly, we shall relate communication to different psychological dimensions of the communicating parties. There seem to be at least three different aspects:

a) the cognitive functions which have to do with knowledge, beliefs, and intellectual understanding. From the expressive point of view communication may be seen as expressing the views and beliefs of the sender, and in an evocative perspective communication is viewed as directed towards 
arousing beliefs, conveying information anc bringing about understanding. Since cognitive activities always have some "intentional objects" - they always "are about" something in the world that the parties believe or want each other to believe - the cognitive and referential functions are heavily intertwined and are seldom kept properly distinct.

b) the emotive functions; in communication the sender expresses his feelings, attitudes, emotions and desires, and this may also evoke the corresponding states and activities in the receiver.

c) the practical functions; much communication is used for guiding the behavior of the receiver, i.e. the messages are conveyed in the hope of arousing a readiness in the receiver to act in certain ways. For obvious reasons, practical and evocative functions often go together. But the sender may also use language for the purpose of planning and guiding his own actions; in that case language is used monologically, and the sender simultaneously plays the part of receiver.

Most linguistic messages are multifunctional, although many are specialized in various ways so as to stress certain particular aspects. However, the actual state of affairs is rather inadequately reflected in most theories of lingulstic meaning, which are very much focussed upon the cognitive and referential aspects. These are the properties which are most independent of particular senders and receivers, and the medium of written communication very strongly emphasizes precisely these aspects. The aim behind certain types of texts is very much to describe reality as explicitly as possible and to display the lines of argumentation as clearly as possible (III.1). The ideal is often to pursue description, explanation and argumentation in such a way that they appear to be independent of the views of the autior (cf. legal and scientific texts). 
In spite of the fact that such objectives are typical of certain forms of written communication, there is also a wide-spread belief that language in general is primarily directed towards the cognitive and referential functions of communication.

All this means that little attention is paid to those functions which often dominate in face-to-face interaction. That is, the expressive, evocative and social functions, i.e. those which are directly related to the communicating subjects, tend to be neglected, and the same holds for the emotive and practical aspects, as opposed to the cognitive side. Notice that these functions are quite dependent on the context at large, on nonverbal communication and prosody (cf. VI.2).

We only need to take a short look at comtemporary work in linguistic semantics to see that only cognitive and referential functions are really considered to be of fundamental linguistic importance. This is, as was just pointed out, in full agreement with the written language bias. It also squares well with the emphasis on individuals and monologues rather than social interactions and dialogues that is typical of much of American psychology and linguistics, and perhaps of western culture in general ${ }^{51}$.

American academic psychology has always had a distinct bias towards individual psychology. This is also true of psycholinguistics, both the variant inspired by generative linguistics and the more recent information processing theories in "cognitive science". One may also recall chomsky's view that language is primarily a means for the expression of thought. The utilization of language in communicative dialogues is to Chomsky more or less an accidental phenomenon (Chomsky 1975:56 ff.). Thus, language is consistently seen as a means for storing, representing, transmitting (transporting) knowledge, not as an ingredient

5) Cf. MacLuhan (1965). See also § III.1. 
in people's social interaction. Since writing is monological rather than dialogical, this is exactly what we should expect.

Among those scholars who have thought deeply about the written language perspective in our Western culture is Walter ong. Here are two pertinent passages:

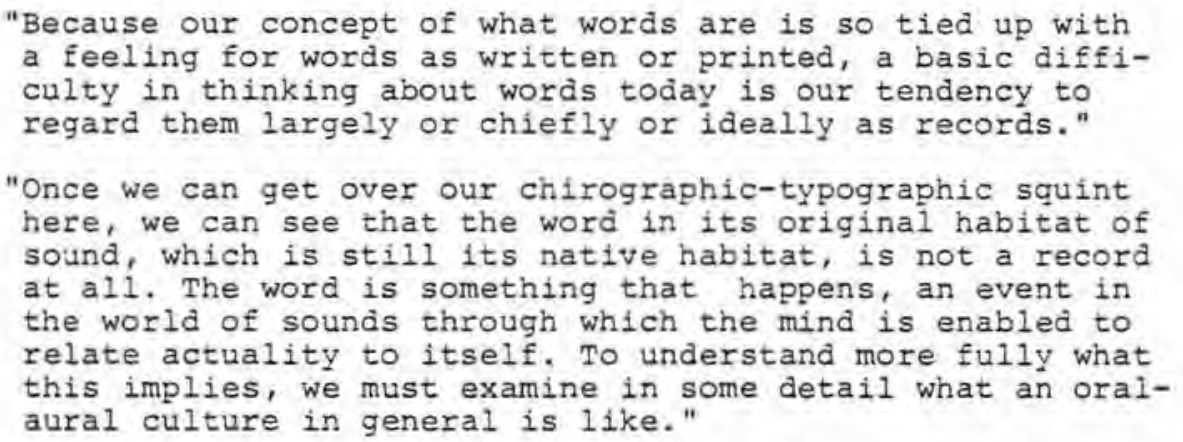

(Ong 1974:167-8)

The functions of words in oral cultures are of course very much emotive and practical. As an additional point, which is not merely a curiosity, one might note here that "primitive" cultures often regard speech as magic. Words have a magical function; one can bring about things by the spell of words; nature and other people may be changed (Ong op.cit.:168-9) .

"Primitive man commonly feels that one can use words to hurt people as one can use an arrow or spear: hence varfous magic formulas."

(Ong 1974:168)

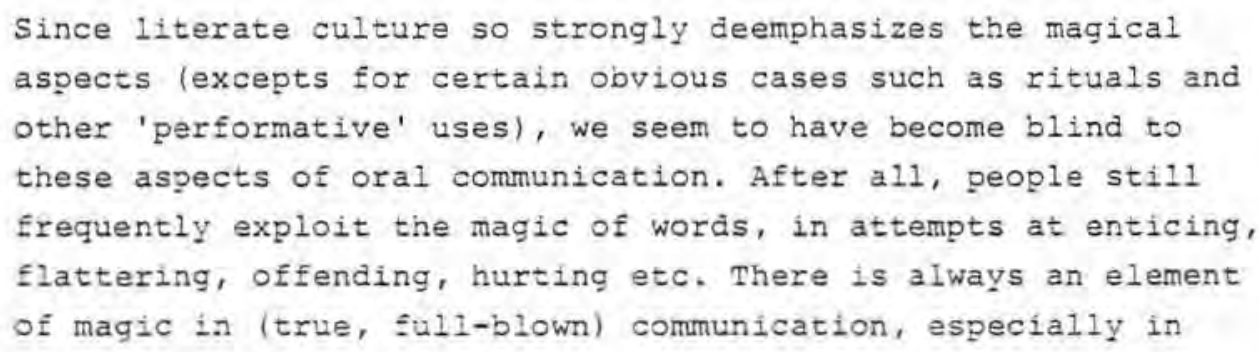


face-to-face interaction, when a speaker - by means of words and non-verbal signals - makes things happen with the listener, when he induces thoughts, images, feelings, moods, and volitions in the listener's mind. Moreover, if we turn to children's play with words, we can see the indissolvable relation between language and social and physical reality. In (symbolic) play children pretend that things happen, they make things happen in the concrete world of play, and this they bring about by the mere use of words (Strömqvist 1980).

\section{IX.3. Thought and expression: Content and form}

Translation theories of linguistic communication suggest a separation between the content and the expression of the linguistic message. Early in the process of utterance production we find the thoughts and "representations" which directly encode or are even equal to the content of the message, and only later the whole message gets transformed into phonetic behavior; the message, and its meaning, is given an outer form. The comprehension process is essentially the reverse; the listener starts with the expression and ends up with the content. These theories are legion in the overwhelming majority of books and articles dealing with utterance production and perception within modern psycholinguistics, but the general ways of thinking are old; the theory of utterance production just sketched may be associated with, among others, Wilhelm Wundt (Gardiner 1932, Blumenthal 1970).

There are three elements in the philosophy underlying translation theories of utterance production (and comprehension) that are worth discussing in this context: 
a) the thesis that speech, or linguistic products in general, are nothing but expressions of thoughts and ideas,

b) the thesis that content and form may be ripped apart as two independent phenomena,

c) the thesis that messages and their meanings or interpretations are mental entities, i.e. things existing in the inner world of our minds (though they presumably have physical substrates in our brains).

Although these theses are interrelated, we shall here deal with them separately.

It is a popular conviction that the process of saying something meaningful involves two steps; first the speaker comes up with and elaborates an idea or thought, and then he expresses this idea linguistically in a verbal utterance. As a general theory 0 discourse production, this is wrong, however, because speakers elaborate their messages through the verbalization process itself, i.e. form and content are created simultaneously. On the other hand, there are of course cases when speakers have prepared their utterances carefully and when they in fact express ideas that have been conceived in advance. An academic lecture or a solemn speech are good examples. In fact, the "translation theory" (IX.1) of discourse production is part and parcel of classical rhetoric, which analyzes the whole process into the discovery and arrangement of ideas ("invention, disposition"), the discovery of appropriate expressions ("elocution"), and then the memorization and actual delivery of the speech (e.g. Beaugrande \& Dressler 1981:15). But the most obvious application of the theory under consideration concerns the expression of messages in writing. Many final written products are very well prepared, and they may appear to be quite careful and explicit expressions of consciously intended messages. (Yet, not even these meticulously compiled texts "contain" their interpretations (cf. VII.6)). The study of such written products may lead the scholar's thinking onto 
wrong paths. He may forget that he is only analyzing the final product of a long series of trials and errors, in which the author only gradually managed to elaborate his points adequate$1 y$.

A more serious error is commited by the linguist, if he believes that the mere analysis of final written products could provide a theory of how thought and language interact in spoken discourse. It is simply misleading to regard speech only as "the use of articulate sound-symbols for the expression of thought" (Gardiner 1975:17). This theory was criticized earlier in $\S$ VI: 1 , when I discussed the traditional definition of the sentence as "the expression of a complete thought". In face-to-face interactions we do not find so much of the expression of complete thoughts (cf. quotation from Gardiner in $\S$ VI.1). On the one hand, thoughts are elaborated and accomplished through, not before, the interaction. On the other hand, we must not put emphasis only on the intellectual side of language; in addition to the referential and cognitive aspects, we have the expressive, evocative, social, emotive and practical aspects which often dominate in spoken dialogues (IX.2).

Our second point is one which has already been touched upon in $\S$ IX.1; thoughts and meanings are often assumed to exist prior to, as it were independent of, the linguistic expressions that encode them in communication. In other words, thought and language are considered to be autonomous and mutually independent in a sense; meanings can be discussed in isolation from their corresponding expressions, the same meanings can be expressed in in many ways and in entirely different languages, and, converse1y, the same linguistic expression can be used with entirely different meanings. Language, in the sense of an expression system, is assumed to be an autonomous, uncontroversial "outer form"; linguistic expressions are seen as containers, empty by themselves but in various communication situations filled with content to be transmitted. Thus, this fits the translation or transportation metaphors in communication theory (IX.1). 
It is quice obvious that the theory just sketched is excreme and unacceptable also in the eyes of most linguists, who do, after all, admit a relation of "solidarity" between the form and content of linguistic signs. Nevertheless, it lurks behind many popular descriptions in linguistic textbooks; we have seen that, e.g., translation theories of communication are legion there. It is fascinating to speculate over the historical origins of these ideas. It seems to me that one possible source may in fact be found in linguists' traditional work with translations between various languages. The ideal here is of course to keep the content constant over different languages; linguistic expressions would then seem to be only the outer appearance. However, translators and interpreters of course know that, in practice, translations are never semantically identical. Translation is a creative reformulation with clear effects on the content. Yet, for various reasons the assumptions of autonomy are more motivated as far as written language is concerned. If we consider spoken dialogues with its bewildering richness of signals, impulses, stimuli and responses, intentions and expectations that are partly private and partly mutually shared and mutually ascribed to one's interlocutor, then it seems that the idea of semantically equivalent expressions is very far-fetched indeed. Meanings and interpretations are created through the activities of verbalization and social interaction themselves.

I have argued that the written language bias in linguistics has promoted the liea of an autonomy of language. However, we have seen that this idea occurs in two rather different forms which seem to lead up to a serious contradition. On the one hand, we have just seen that arguments have been made for the autonomy of linguistic expression with respect to linguistic meaning (and vice versa). On the other hand, we found in § VIII.10 that the idea of language as an autonomous and primary force in mental life has engendered the hypothesis of linguistic determinism, which in fact assumes the link between expression and meaning to be very stable and rigid indeed. Thus, 
these ideas seem to be entirely inconsistent. However, attempts have been made, e.g. in generative linguistics, to reconcile the two opposing views. Thus, this theory assumes, on the one hand, that linguistic expressions as surface structures are very indirectly related to thought and meaning (the autonomy of linguistic expressions), while, on the other hand, deep structures, or semantic representations, are very closely related to thinking (which amounts to a specific variant of linguistic determinism). The thesis of the autonomy and independence of language is therefore taken to an extreme in Chomskyan linguistics (cf. also V:5-6, X).

The third main point of this section is the idea that meanings are mental things in the minds of language users. In VIII.3 I suggested how such a conclusion may be arrived at. That is, the argument there was concerned with images that had to do with expressions (sound images), but similar arguments can be made as regards meaning. The philosophical problems would also be much the same.

The "intentional objects" of thoughts and verbal utterances, i.e. that which we think or talk about, are, after all, usually extralinguistic phenomena in the physical and/or social reality around us. For example, if someone tinks of or talks about Big Ben in London, then it is the "real" Big Ben which is the intentional object, not an image of Big Ben or any other mental object in the speaker's mind. It is another matter that any given speaker at any given occasion must talk about Big Ben under a certain aspect, which means that the speaker always "contributes" something to the way he thinks or talks about it ${ }^{6)}$. on the face of it, the most puzzling phenomenon about thinking and talking may be the fact that we can use non-existing things

6) The distinction between the distal stimulus and the proximal stimulus in the theory of perception (of. e.g. Fodor $1976: 55)$ seems parallel to this distinction between "the referent" and "the (same) referent under a certain aspect". 
as intentional objects. Suppose we think about a unicorn; where is the intentional object of such a cognitive act? Some may be tempted to answer that the unicorn is a mental thing that exists in the subject's mind. However, in my view this leads to unsolvable problems. The only reasonable answer is that, to the best of our knowledge, no real counterpart of such an intentional object exists. In other words, our question was wrongly put. This is not to deny that the act of thinking is real enough, or that we have a subjective experience of seeing a unicorn in our "inner eye". The important thing is to distinguish between acts of thinking and speaking (and conditions, schemas, background knowledge etc involved in such acts), and the intentional objects of these activities; the latter may be objectively nonexisting just as an image according to optical theory may be only virtual.

IX.4. Social and individual aspects of language.

It is characteristic of wundt and a long tradition in linguistics (IX.1) that the direct associations between thinking and meaning, on the one hand, and linguistic form on the other are cut off. Thoughts are assumed to exist independent of and prior to language; first we think and organize meaning, then we speak and transfer the message! But this is not the only unfortunate separation in traditional linguistic philosophy; another separation, inherent in e.g. Saussure's distinction between language and parole, is that between the social and individual aspects of language and its use:

"En séparant la langue de la parole, on sépare du même coup: $1^{\circ}$ ce qui est social de ce qui est individuel; $2^{\circ}$ ce qui est essentiel de de qui est accessoire et plus ou moins accidentel."

(Saussure 1964:30)

In this theory, "language stands in opposition to utterance in the same way as does that which is soctal to that which is indi- 
vidual" (Volosinov 1973:60). On the one hand, we have the social system of stable signs, i.e. la langue, on the other hand we have the individual's use of this language, which is then conceived of as a more less accidental use of ready-made units, i.e. words and phrases with fixed meanings.

Such a theory is entirely inadequate as a background for understanding spoken dialogues. Communication in normal speech situations is an inherently social enterprise, a complex interaction in which the various moves of the agents involved can never be understood from a purely individual perspective. Nor can the social aspects be entirely relegated to the domain of prefabricated social rules (la langue and the like). Speakers and listeners participate in social interaction, and so do writers and readers, although in a much less conspicuous way. In fact, if we consider written communication, Saussure's attempt to derive the social/individual distinction from the underlying differences between language and the use of language, becomes much more natural. Both writer and reader normally work alone, thus seemingly performing individual (monologic) activities, and in doing so, they apply the rules of a language system that is socially shared and normatively standardized to a greater extent than is the case in speech communication. Thus, Saussure's abstract objectivism $^{7)}$ (as regards his conception of la langue)

7) Vološinov (1973) actually identifies two rather different trends in western philosophy of language. One is the abovementioned abstract objectivism (language as a stable, immobile system of normatively identical forms which are presented to the individual user as ready-made signs), which has no doubt been the most prominent zradition. The other one is individualistic subjectivism, which volosinov associates with Humboldt, Vossler, Leo Spitzer, Croce and others. This tradition looks upon language as activity, as an unceasing process of creation realized in individual speech acts. This tradition thus puts emphasis on the novelty of each individual speaker's emotions, thoughts, impulses, ambitions, intentions etc. It seems to me that the focus on the individual's creativity and on his mental activities is achieved very much at the cost of ignoring the social interaction in dialogues. Ultimately, Vossler's approach owes a lot to stilistics, rhetoric, the analysis of belles lettres etc. and these disciplines have been mainly concerned with the monologic use of language, 1.e. with artistic, very elaborated and elegant, consciously and meticulously planned utterances and texts. Such linguistic products are after all more typical of written communication than spontaneous speech. Thus, even this tradition turns out to be rooted in the analysis of written language. 
and his view of linguistic performance reflect a bias towards written language, in spite of his own ardent defense of the primacy of spoken language (see v,2).

\section{IX.5. Semiotics}

Semiotics is usually defined as the study of signs. Communication is necessarily based on various forms of signing and signalling, and semiotics is therefore, at least potentially, of vital interest for communication theory.

It is customary and appropriate to distinguish between the European tradition of semiotics founded by Saussure and an American variant going back to Ch.S. Peirce. Saussurean semiotics for semiology, as his term was) has been deliberately based on structuralist models of language structure. It is therefore no wonder that this kind of approach to communication is strongly 1y biased by the written language perspective in linguistics.

What is perhaps most characteristic of Saussurean semiotics is the conception of a sign as an association of two thing-like phenomena, expression and content. The linguistic sign, we recall, was construed as a more or less stable combination of a mental representation of the sound signal (image acoustique) and a "concept", and the latter is also a mertal representation of some kind. This seems to be a generalization at a somewhat more abstract level of how linguistic expression and meaning are sometimes represented in the written medium; the expression is given as a graphic word (or a combination of such words), and the meaning is portrayed, if possible, as a picture of the thing(s) meant.

The philosophical difficulties inherent in the theory of mental representations and images have already been alluded to. But there are other features of Saussurean semiotics which are 
rather unsatisfactory, especially when applied to communication through face-to-face interaction. The most obvious flaw is the neglect of the communicators, the sender and the listener. Whereas American semiotics does comprise also the pragmatic dimension, Saussurean semiotics does not really tackle the problems of dynamic interaction in communication. It is an approach which entirely focusses on the underlying system of signs, and this is never sufficient if we want to understand communication, i.e. the use of signs for various purposes in different situations. Accordingly, classical European semiotics tends to describe communication as an exchange of various stable signs (cf. communication as a transportation of fixed messages).

To be sure, there are semiotic approaches which are less tied to extreme structuralism. As we have already noted, Peirce's pragmatist semiotics seems better equipped to tackle the interplay of signs and symbols in face-to-face communication. Unlike Saussurean semiotics it is not an extension of linguistic modes of analysis, but is based on more behaviorally oriented traditions in American philosophy.

There are also some modern semioticians in Europe who have realized that utterances, texts or human acts in general cannot be ascribed unique and stable meanings. Thus, Julia Kristeva (1969) stresses the manifoldness of interpretations; she prefers to analyze texts in terms of acts and processes, or, more precisely, in terms of meaning-creating activities, The production and assignment of meanings must be seen as praxis; the communicating parties work with the creation of meanings and interpretations in the production of situated, (partially) shared understandings, they do not simply function as passive sources and goals for a transportation of messages. 


\section{LANGUAGe acQuISITION}

Our theories of language acquisition will naturally be dependent on what theories we have of language structure and language use. Accordingly, if we think of language as an autonomous structure which is relatively independent of other sign systems (non-verbal communication) and situational contexts, then we do not expect, of course, that the properties of language could be explained by reference to such phenomena. Exactly this predicament characterizes some structuralist approaches to language acquisition; they look at the ontogenesis of language as something which requires very special explanations, i.e. explanatory principles which are good only for language. Let me illustrate this by calling attention to some features of the theories of two great structuralists, Roman Jakobson and Noam Chomsky.

\section{X.1. Phonological development}

Roman Jakobson is famous for, among other things, his very clearout distinction between prephonological articulatory and perceptual developments in the child, which, in his opinion, has nothing to do with language, and phonological development, which is part of language acquisition. Thus, Jakobson argues that babbling is relatively unstructured, that it contains all sorts of phonetic effects, and that it has no relation whatsoever to the later phonological development. The latter, on the other hand, exhibits a universally valid structure in that children develop phonemic constrasts in very regular ways, from the most primitive system of just two or three units to the full-blown system of the adult language. Whereas phonological development is concerned with the acquisition of the distinctive features of the symbolic system, i.e. language, babbling has no such functions at all. 
logical distinctive functions and the more or less continuous vocal behavior of babbling seems absolute. However, it may easily be wrongly stated, and it has de facto served to make linguists less eager to look at the structure of babbling. In fact, the child's phonetic achievements in the babbling period and the early stages of language acquisition are not unrelated. Babbling is not completely chaotic; rather it displays preferences for certain phonetic structures, at many points those which Jakobson predicted should appear early in the phonological development (01ler et al 1976). MacNeilage (1979:30) observes:

"From this viewpoint the child's first words can be seen as, at least partially, a matter of choosing from the babbling repertoire a set of approximations to adult word forms"

If, furthermore, we want to investigate young children's ways of phonetically distinguishing recognizable words belonging to the language they are surrounded by, then it turns out to be impossible to observe the early stages of phonological development that Jakobson postulated, since children have a considerable repertory of phonetic units, and hence many potentially phonemic distinctions, already at the stage when they have a vocabulary of say 25-30 words, i.e. at the earliest point in time that it is possible to perform any phonological systematization. On the other hand, some more primitive stages in the phonetic development may be observed in babbling. Thus, the development from prephonological to phonological stages is gradual rather than absolute.

Moreover, Jakobson's neglect of babbling and other early vocal and non-vocal behaviors is unfortunate, since such behavior has important emotive, social, evocative and expressive functions. These are functions which are still dominant, as the child goes on to develop language. If we want a comprehensive perspective on the child's commulcation capacities, the development from prelinguistic to linguistic means of communication is gradual and far from linguistically uninteresting. Instead, 
we can see that the isolation of language as a communication system that is considered to be absolutely unique tout court is a reflection of the linguist's tradition of considering mainly or only the cognitive and referential functions in communication (IX.2).

\section{X.2. The innate faculty of language}

Since the mid 1970's there has been a vigorous movement in child language studies attempting to look for sources and similarities of spoken utterances and speech acts in the non-verbal communicative acts that occur very early in the child's life and develop into more and more diversified forms of interaction as the child grows older. Scholars (e.g. Werner \& Kaplan 1963, Bruner 1975, Greenfield \& Smith 1976, Bates et al 1979) have been able to show that there are interesting relationships between the non-verbal acts preceding language acquisition and the later communicative acts that comprise both verbal and nonverbal aspects. This applies both to the action-theoretical status (functions) of singular acts and to their internal semantic and formal structures. Certain fundamental features of syntactic organization seem to reflect properties of basic action patterns; Bruner has argued in this vein with regard to both the topic-comment structure and the role structure of sentences ("role" in the sense of Eillmore's deep case or the like).

Thus, the scientific approaches just mentioned serve to establish connections between language and various non-verbal communication systems. This goes against traditional linguistic yiews that assign an absolutely unique position to language. Perhaps the most extreme structuralist view in this regard is Chomsky's flat denial that there are any interesting relations at all between language and more "primitive" symbol systems le.g. Chomsky 1968:70 ff.). Chomsky's position, however, is quite logical, given the long-time goal of establishing linguistics 
as an independent discipline with a purportedly well-defined object of study, i.e. language as an autonomous system. Chomsky in fact ascribes to the ideal speaker's language a very intricate structure, such as could be realistically described only by a generative transformational grammar. If language users have indeed "internalized" such grammars, then it seems inconceivable, says Chomsky, that all of this could be explained as developments of non-verbal symbol systems. Accordingly, Chomsky hypothesizes that man has an innate highly specialized capacity for language, a very specific faculty (facultê de langage) independent of other faculties of the mind. That is, he postulates a biological basis for language in a way that precludes the possibility of deriving properties of linguistic capacities from other cognitive abilities and social skills. This metaphysics is unfortunate at two levels. For one thing, the postulation of a specific capacity for only language is ad hoc and therefore methodologically inferior in that it persuades linguists not to seek for deeper explanations (Derwing 1973:63 ff.). Secondly, it is in fact at least partly unmotivated on empirical grounds, because the above-mentioned research on child language seems to show that there are lots of significant relationships between non-verbal and verbal means of communication. However, all this presupposes that we really explore into the dynamic interaction of spoken discourse. This is something that generative linguistics does not do. As we have seen (IX.2) Chomsky even argues that communication is an accidental property of the use of language! 
In any society, but particularly so in a modern industrialized society with a lot of professional specialization, there is a great deal of linguistic variation. First of all, there are of course the overall differences between written and spoken language. In addition, however, there are many kinds of variations within the range of written language and, in particular, within the range of spoken varieties. The written language bias in linguistics has several kinds of repercussions on the linguists' ways of handling this variation. On the one hand, the fundamental attitude was for a very long time one of ignoring the variations (XI.1). On the other hand, to the extent that different varieties are indeed attended to, one finds certain characteristic evaluations of them that are clearly reminiscent of the long tradition of considering standard written language as the only proper, correct language (XI.2).

\section{XI, 1. The neglect of linguistic variation}

After all, linguists have most often worked with made-up linguistic examples (usually written word forms and sentences) applying their linguistic intuition for gramaticality etc to them. To the extent that the analyses have been based on corpuses of language actually used for communication, the data have until rather recently been drawn almost exclusively from written texts. Most of the linguistic variation of spoken language was therefore never attended to in any systematic fashion. The prevailing attitudes were neglect and ignorance.

One common attitude among linguists is, or was until recently, to regard the linguistic variation in speech as more or less chaotic and devoid of any interesting and "linguistically significant" regularities. After all, language was conceived of as the system of underlying context-independent invariants, and the norms underlying invariants are based mainly on 
written language. The linguists' attitudes towards variation are (or were) characterized by a lack of attention and interest; all variation, at least if it is not derivable from structural factors (so-called combinatory variation), is (or was) termed "free" and described, if at all, by "optional rules", which are assumed to apply in an essentially random fashion; hence, no regular patterns are generated.

Moreover, it is customary to ascribe variations in speech to "linguistically irrelevant" factors such as lack of knowledge (on the part of speakers and listeners), shifts of attention, fatigue, memory limitations and other disturbing factors in face-to-face interaction. Accordingly, one arrives at a prejudiced view of what normal spoken language is like. Research into child language and "baby talk" (the speech of adults directed towards children) has shown in recent years that Chomsky's description of the child's predicament is highly inaccurate:

"Thus, it is clear that the language each person acquires is a rich and complex construction hopelessly underdetermined by the fragmentary evidence available."

\title{
(Chomsky 1975:10)
}

"Knowledge arises on the basis of very scattered and inadequate data and ... there are uniformities in what is learned that are in no way unicuely determined by the data itself."

(Chomsky 1966:65)

\begin{abstract}
"A consideration of the character of the grammar that is acquired, the degenerate quality and narrowly limited extent of the available data, the striking uniformity of the resulting grammars, and their independence of intelligence, motivation, and emotional state, over wide ranges of variation, leave little hope that much of the structure of the language can be learned by an organism initially uninformed as to its general character."
\end{abstract}

$$
\text { (Chomsky 1965:58) }{ }^{1)}
$$

1) The emphasis is mine (PL). Note that Chomsky makes the highly implausible assumption that the child internalizes something like a generative transformational grammar when it acquires its language. For critical discussion, see Derwing (1973), tinel1 (1979), and references there. 
It is true that language use is heterogeneous in many ways. But it is still orderly and structured, and a considerable amount of the regularities are conventional and specific for the language involved and its varieties. Iinguistic "performance" may seem chaotic, but most aspects of reality do so as long as they have not been systematically investigated. In fact, a great deal of variation in speech performance is regular and patterned; the different variants are systematically favored or disfavored by various contextual factors (linguistic-structural, psychological, social, cultural etc.). In recent years such facts have been unearthed by proponents of so-called variational Iinguistics, who have analyzed phenomena as different as normal dialectal variation (Labov 1969, Cedergren \& Sankoff 1974), pidgins and creole languages (DeCamp 1971), and variation in the interlanguages of second-language learners (Bickerton 1971, Hyltenstam 1978).

The fact that variations in linguistic performance are usually ignored by linguists and psychologists may also lead to an implicit assumption that the existing variations are not too extensive after all; one assumes that speakers, listeners and readers are very much alike as regards what they can produce, perceive and understand, and what strategies and linguistic operations they apply in performance. In the psychology of language use and reading we find many models of utterance production, utterance comprehension, and reading with universalistic pretentions. It is hoped that one can construct models of utterance planning, reading etc that are more or less independent of all the varying goals and expectations of different communication situations. Contrary to what such general models suggest, it seems likely that a great deal of our use of language and linguistic knowledge is task-oriented; looking for a number in the phone book and reading a poem by Wordsworth are, after all, quite different activities. Everyday small talk and academic discussions on complicated topics are in many ways rather different in character. Therefore, it remains to be seen what the common components of linguistic processing, if any, are. 
However, linguistic performance does not only vary with the different communicative tasks. It also depends on the linguistic habits and knowledge of the persons involved. Here again a common attitude among psycholinguists and phoneticians is that all speakers and listeners behave in roughly the same way. Therefore, linguists and phoneticians are very often satisfied with using only a few subjects in their experiments. It seems to me that we are encouraged to consider this a safe strategy, because as linguists we entertain certain views of language and language users. Language is assumed to be a monolithic, stable and homogeneous system, and normal language users are assumed to share this system to a great extent $(V: 5)$. However, we would need a lot more of differential phonetics and psycholinguistics before we can safely state that native language users are indeed alike in their speaking, listening and reading strategies.

\section{XI.2. The depreciation of spoken vernaculars}

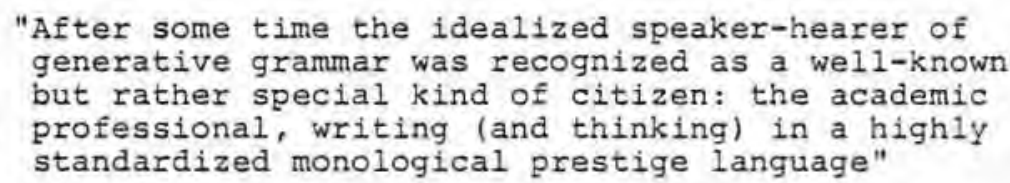

(Teleman 1980:335)

The next point concerns the ways in which spoken and written varieties are evaluated with regard to correctness and social status. Therefore we shall return to the theme of $\$$ III.3, the traditionaliy high status of written standards in most societal contexts, and the ways in which this has influenced scientific thinking in linguisties.

The linguistic situation of many societies is best characterized in terms of diglossia (Ferguson 1959), the existence of two languages or linguistic varieties having different status. There is a high status language used in official contexts, such as 
legislation and jurisdiction, administration, higher education, religious services. This language is primarily used in writing, and if or when it is used in speaking, it is typically in situations where one reads aloud messages which exist beforehand in written form. This language is sometimes quite remote from the living spoken language of everyday life (different grammar and vocabulary, different functions). The latter everyday language is typically a low status language. It nay be used in writing too, but, if so, primarily in non-official and nonformal contexts, such as personal letters, folk literature, popular newspapers etc. In some cases the written high status language is partly archaic and based on features which are considered part of an ancient cultural heritage. A good example would be Greek katharevusa, the literary and constitutional language of Greece, largely based on previous forms of literary Greek. Until recently (1976), this was the official language of Greece, in spite of the fact that for a long time dhimotiki was the only language naturally used by the majority of the people. Incidentally, one may note that the literal translation of katharevusa would be "pure language", while dhimotiki is "the people's language".

Even in societies where diglossia is not so salient at a first glance, most people usually regard spoken vernaculars as worth less, as being less correct and less efficient than written language. What is characteristic of spoken language is often regarded as deviations from "proper language", and proper usage is again that which is prescribed by written standards. A typical statement (about Swedish) would be:

\footnotetext{
"Man säger längre än mig, men egentligen heter det längre ün jag" (trans.; We say taller than me, but actually it should be taller than I)
}

Thus, the implicit norms of spoken language are often simpiy incorrect in the ears of the public. Similarly, variants which deviate grossly from national standard languages, e.g. very strong "accents" (highly "deviant" geographical and/or social 
dialects) and child language, are simply considered downright wrong. These variants almost always lack written counterparts. ${ }^{2)}$

An interesting case in point is the interpretations given to Basil Bernstein's concepts of "elaborate code" and "restricted code". I am not going to discuss all the interpretations which have been made, justifiably or (most often) unjustifiably, in the debate initiated by Bernstein, but there are certain recurrent features which deserves mention. First of all, the choice of terms, i.e. "elaborated" vs "restricted", carries with it certain obligatory connotations. The formal elaboration typical of written language is regarded as better, more adequate, more logical, and less limited than the structure of the language of everyday experience and everyday tasks, i.e. linguistic varieties that are most typical of communicative interaction in everyday non-official private situations. Accordingly, some commentators have associated Bernstein's notion of elaborated and restricted codes with the decontextualized vs context-bound forms of written and oral discourse, respectively (cf. Kay 1977).

Furthermore, a decade ago, some of Bernstein's commentators and eclectics, as well as other educators, particularly perhaps in the U.S.A. (e.g. Bereiter \& Engelmann 1966), argued quite strongly that many colloquial dialects, e.g. Black English, were somehow deficient and lacking in grammatical structure, and that they reflected an unsystematic thinking, thus impeding further cognitive and cultural development on the part of the "linguistically deprived" children and youths. Of course, these preconceptions should by now be disproved (e.g. Labov's (1969) forceful arguments), but they provided yet another example of

2) Naturally, child language is "deviant" or "incorrect" when compared with the goal of language leaming, which consists in the internalization of aduit language. However, this is not the only perspective that could or should be applied to children's linguistic productions. For certain purposes it is clearly desirable to consider children's language as a linguistic system with its own rules and principles. 
the common attitudes towards colloquial dialects as compared with standard (written) languages.

The belief that formal elaboration, which is the ideal of certain written genres, would in itself lead to a more correct and communicatively more forceful language is of course a myth, but it is a myth which is very deeply rooted in our entire culture. That everyday spoken language is plain and poor, deficient, illogical, incoherent and ungrammatical is something which has been part and parcel of school education for millennia. Children should for this reason receive a correct and decent language at school. For many centuries it was thought that this activity of cultivating and refining the pupil's language was naturally related to the civilizing correction of his crude and evil morals. Martin Luther is one of those who have expressed this explicitly:

\footnotetext{
"Des lasst uns das elend greulich Exempel zur Beweisung und Warnung nehmen in den hohen Schulen und Klöstern, darin man nicht allein das Evangelium verlernet, sondern auch lateinische und deutsche sprache verderbet hat, dass die elenden Leute schier zu lauter Bestien worden sind, weder deutsch noch lateinisch recht zu reden oder schreiben können; und beinahe auch die natürliche vernunft verloren haben."
}

(Martin Luther quoted by Diderichsen 1968:19)

Here is a translation from a regulation for the Swedish school system ecited in 1649 (cf. Thavenius 1981:138):

\footnotetext{
"It is true that the boys carry their mother tongue with them from home but it is most often crude and uncultivated, sometimes even filthy and spoilt. Therefore it needs to be cultivated and refined at school, and teachers should take pains to teach the pupils, each according to his age, how to speak and write his mother tongue in a good and cultivated way. Teachers will find opportunities for this in several ways but particularly much in translating and reproducing the Latin authors and in correcting the boys' own translations."
} 
As we have noted earlier (III.3), school education served to emphasize the social barriers between the very few, who had access to the written language, and the vast majority of the people, those who were living in their everyday oral culture:

\begin{abstract}
"The grammatical distinctness and the formulaic nature of literary styles along with the many highly artificial calligraphic conventions such as those illustrated in W.J. Ong's "Latin Language Study as a Renaissance Puberty Rite" (1959), tend to function as access barriers which kept schooling and hence command over litery skills confined to small groups where learning was more a matter of personal, tutorial type contact between students and teachers and informal socialization by small group process than of formal curriculum. For those not born as members of literate groups who succeeded in crossing these barriers, becoming literate meant loosening familial ties to taking on a new cultural identity."
\end{abstract}

(Cook-Gumperz \& Gumperz 1981:94)

Our views on education and schooling have undoubtedly changed considerably since the sixteenth and seventeenth centurtes, but there are certain features in the views on language which prevail from Luther down to Chomsky. Although Chomsky obviously belongs to quite another context, it remains true that he too argues that normal spoken discourse is faulty, deficient, and ungrammatical (XI.2). It is indeed true that written standard language is constrained by more explicit norms and is therefore characterized by a more stable grammatical structure than most types of spoken language, but the most important difference clearly lies in the fact that spoken language is subject to other rules, and this in turn has at least partly to do with the fact that spoken language belongs to cultural contexts of use which are different from those of written language. 


\section{XI.3. The concept of an ideal language}

Due to the lack of an immediate context, most written messages have to be relatively explicit and relatively autonomous. In many genres it is clearly part of an ideal goal to write as explicitly as possible. The acquaintance with the impersonal, objective, and explicit written medium has encouraged several mathematicians, logicians, and some linguists to adopt a much higher goal, indeed the ultimate goal of inventing the absolute1y explicit and autonomous language, a language in which every form and every grammatical operation has its absolutely fixed and stable interpretation. No additional intuition or lmagination, no subsidiary knowledge on the part of the individual user would be needed in order to understand messages coded in this language. Thus, texts coded in this language would be absoluteIy autonomous, 1.e. their interpretations would be entirely inherent in the texts. It would be a language of exact thinking, a universal language, immaculate and unfettered by all the historical accidentals that have produced the differences and idiosyncracies of natural languages and the everyday use of them.

This is the dream of an ideal language entertained by Leibniz and other philosophers. It is interesting to see what kind of language was considered to be ideal: an absolute idealization of written language. However, such a language would be far from ideal in reality, in fact it would be entirely useless as a language in particular for spoken discourse and social interaction. There we need a language which is powerful and flexible enough to adjust to the infinite multitudes of situations and interpretations that human beings may encounter and invent. Such a language must not determine interpretations, it may only allude to them (VII.1 ff.). 
XII, EPILOGUE

We have come to the end. Clearly I have not exhausted my topic; there are more areas where the impact of the written language bias could be seen. Naturally, I am aware that some of my points are not too strong, as I stated already at the outset (IV). However, to me the overall picture is more important, since it provides the setting within which the individual points should be understood.

Having used the word "bias" rather often in this book, I realize, of course, that my own perspective will also be perceived as biased. A reviewer of an earlier version of my text remarked that I seem to have "used the written language bias theme as a vehicle for the expression of views on a host of topics in general linguistics". There is obviously a grain of truth in this. My original impetus came from an interest in the analysis of spoken language and an insight that many basic features of modern linguistics appear to be unsatisfactory when applied to spoken language. Therefore, my book has indeed a clear bias; it consistently views aspects of the language sciences from only one perspective, i.e. that of interpreting these aspects as indicating an influence from the linguists' tradition of analyzing written language. It is nowhere denied, however, that other explanations can be provided too, but these matters must be pursued (and are in fact pursued) elsewhere. My aim has been to invoke some discussion on the topic of the written language bias. I am confident that such a discussion will counterbalance the inherent onesidedness of this book. 
BIBLIOGRAPHY

Allen, J, \& Perrault, R. 1980. Analyzing Intention in Utterances. Artificial Intelligence, 15:143-178.

Allwood, J. 1981. On the Distinctions between Semantics and Pragmatics. In klein, W. \& Levelt, W. leds.l. Crossing the Boundaries in Linguistics. Dordrecht: Reidel, 177-189.

Andersen, H. 1969. A study in diachronic morphonemics: the Ukrainian prefixes. Language, 45:807-30.

Anderson, J.R. 1978, Arguments Concerning Representations for Mental Imagery. Psychological Review, 85:249-277.

Andersson, L-G. 1975. Form and Function of Subordinate Clauses. Göteborg: Dept. of Linguistics.

Bailey, Ch.-J. 1979. Invited comments on congress reports by Basb $\phi 11$, Anderson and Bybee. Ninth Int. Congr. of Phon. Sciences, Copenhagen.

Baron, N. 1981. Speech, Writing, and Sign. A Functional View of Linguistic Representation. Bloomington: Indiana University Press.

Bates, E. et al. 1979. The Emergence of Symbols: Cognition and Communication in Infancy. New York etc.: Academic Press.

Beaugrande, R. de. 1979. New Trends in the Study of Conversation. LAUT.

Beaugrande, $\mathbb{R}$. de \& Dressler, W. 1981. Introduction to Text Linguistics. London: Longman. 
Bereiter, C. \& Engelmann, S. 1966. Teaching Disadvantaged Children in the Pre-School. Englewood Cliffs, N.J.: Prentice Hall.

Berger, P. \& Luckmann, Th. 1967. The Social Construction of Reality. Harmondsworth: Penguin.

Bickerton, D. 1971. Inherent Variability and Variable Rules. Foundations of Lanquage, $7: 457-492$.

Birdwhistell, R.L. 1970, Kinesics and Context. Philadelphia: University of Pennsylvania Press.

Bloomfield, L. 1933. Language, London: Allen \& Unwin.

Blumenthal, A. 1970. Language and Psychology. Historical Aspects of Psycholinguistics. New York: Harcourt Brace Jovanovich.

Boden, M. 1977. Artificial Intelligence and Natural Man. Brighton: The Harvester Press.

Botha, R,P. 1973. The Justification of Linguistic Hypotheses. (In collaboration with $w$. Winckler). The Hague: Mouton.

Botha, R.P. 1979. Methodological Bases of a Progressive Mentalism. (Stellenbosch Papers in Linguistics, 3) (Also in Synthese, 43, $1980)$.

Eruner, J. 1975. The Ontogenesis of Speech Acts. Journal of Child Language 2:1-19.

Butterworth, B. 1980 (ed.) Language Production Vol. 1: Speech and Talk. London: Academic Press.

Cedergren, H.J. \& Sankoff, D. 1974. Variable Rules: Performance as a Statistical Reflection of Competence. Lanquage, 50:333-55. 
Chafe, W. 1979. The Flow of Thought and the Flow of Language. In Givón, I. (ed.). Syntax and Semantics, Vol. 12. New York: Academic Press. 159-181.

Chomsky, N. 1957. Syntactic Structures. The Hague: Mouton.

Chomsky, N. 1965. Aspects of the Theory of Syntax. Cambridge, Mass.: The M.I.T. Press.

Chomsky, N. 1966. Cartesian Linguistics. New York: Harper \& Row.

Chomsky, N. 1975. Reflections on Language. Glasgow: Fontana/ collins.

Chomsky, N. \& Halle, M. 1968. The Sound Pattern of English. New York: Harper \& Row.

Cook-Gumperz, J. \& Gumperz, J. 1981. From Oral to Written Culture: The Transition to Literacy. In Whiteman (1981): 89-109.

Cooper, L.A. 1975. Mental Rotation of Random Two-Dimensioned Shapes. Cognitive Psychology, 7:20-43.

Cooper, W.E. 1980. Syntactic-to Phonetic Coding. In Butterworth $(1980): 297-333$.

Dah1, 6. 1980. Some arguments for higher nodes in syntax: a reply to Fudson's 'Constituency and dependency'. Linguistics, $18: 485-88$.

Daniloff, R.G. \& Hammarberg, R. 1973. On Defining Coarticulation. Journal of Phonetics, 1:239-248.

DeCamp, D. 1971. Towards a Generative Analysis of a Post-Creole Speech Continuum. In Hymes, D. (ed.) Pidginization and Creolization of Languages. London: Cambridge University Press. 
Denes, P. \& Pinson, E. 1963, The Speech Chain. Baltimore: Bell Telephone Laboratories.

Derrida, J. 1967a. L'écriture et la différence. Paris: Coliection "Tel quel". (English translation: Writing and Difference. London: Routledge \& Kegan Paul. 1978).

Derrida, J. 1967b, De la grammatologie. Paris: Collection Critique.

Derwing, B. 1973. Transformational Grammar as a Theory of Language Acquisition. (Cambride Studies in Linguistics, 10) Cambridge: Cambridge University Press.

Derwing, B, 1979. Against Autonomous Linguistics. In Perry (1979):163-189.

Diderichsen, P. 1968. Sprogsyn og sproglig opdragelse. Udg. af Niels Rosenkjaer. Kфbenhavn: Nyt Nordisk Forlag Arnold Buck.

Dijk, T. van. 1972. Some Aspects of Text Grammars. The Hague: Mouton.

Di Pietro, R. 1976. The Strategies of Language Use. In Reich, P.A. (ed.). The Second LACUS Forun, 1975. Colombia, S.C.: Hornbeam Press.

Donaldson, M. 1978, Children's Minds. London.

Donnellan, K. 1971. Reference and Definite Descriptions. In Steinberg, D. \& Jakobovits, L. (eds.) Semantics. An Interdisciplinary Reader in Philosophy, Linguistics and Psychology. Cambridge: Cambridge University Press, 100-114.

Dreyfus, H. 1972. What Computers Can't Do. A Critique of Artificial Reason. New York: Harper \& Row.

Ducrot, 0. 1972. Dire et ne pas dire. Principes de semantique linguistique. Paris: Hermann. 
Duncan, S. Jr. 1979. Face-to-Face Interaction. In Aaronson \& Rieber (eds.) Psycholinguistic Research: Implications and Applications. Hillsdale, N.J.: Lawrence Erlbaum, 357-392.

Ejerhed, E. 1979. Linguistic Aspects of Time in Semantic Memory. Univ, of Umeå: Dept. of Linguistics.

Engdah1, E. 1979. Wh Constructions in Swedish and the Relevance of Subjacency. NELS X, Cahiers Linguistiques d'Ottawa.

Engdahl, E. \& Ejerhed, E. 1982. (eds.) Readings on Unbounded Dependencies in Scandinavian Languages. (Acta Universitatis Umensis, Umea studies in the Humanities, 43). Umead: Almqvist \& Wiksell International.

Ferguson, Ch. 1959. Diglossia. Word, 15:325-40. Also in Giglioli, P.P. (ed.) (1972) Language and Social Context. Harmondsworth: Penguin Books, 232-251.

Fillmore, Ch. 1972. On Generativity. In Peters, S, led.) Goals of Iinguistic Theory. Englewood Cliffs, N.J.: Prentice-Hall, $1-19$.

Eodor, J.A. 1976. The Language of Thought. Hassocks: The Harvester Press.

Fodor, J.A., Bever, T.G. \& Garrett, M. 1974. The Psychology of Language: An Introduction to Psycholinguistics and Generative Grammar. New York: McGraw-Hill.

Foley, J. 1965. Prothesis in the Latin Verb sum. Language, $41: 59-54$.

Fónagy, I. 1977. Le statut de la phonostylistique, Phonetica, $34: 1-18$. 
Fortescue, M. 1979. Why the Language of Thought is not a Language: Some Inconsistencies of the Computer Analogy of Thought. Journal of Pragmatics, 3:67-80.

Foss, D. \& Hakes, D. 1978. Psycholinguistics: An Introduction to the Psychology of Language. Englewood Cliffs, N.J.: Prentice-Ha11.

Fowler, C. 1980. Coarticulation and Theorles of Extrinsic Timing. Journal of Phonetics, $8: 113-133$.

Gardiner, A. 1951 (1932). The Theory of Speech and Language. (second ed.) Oxford: Oxford University Press.

Garrett, M.F. 1980. Levels of Processing in Sentence Production. In Butterworth $(1980): 177-220$.

Gazdar, G. 1980. Pragmatic Constraints on Production. In Butterworth, (1980): 49-68.

Goldman-Eisler, F. 1968. Psycholinguistics: Experiments in Spontaneous Speech. London: Academic Press.

Goody, J. 1977. The Domestication of the Savage Mind. Cambridge: Cambridge University Press.

Goody, J. \& Watt, I. 1972. The Consequences of Iiteracy. In Giglioli, P.P. (ed.) Language and Social Context.Harmondsworth: Penguin, 311-357.

Greenfield, P.M, \& Smith, J.H. 1976. The Structure of Communication in Early Language Development. New York: Academic Press.

Grice, H.P. 1975. Logic and conversation. In Cole. P. \& Morgan, J.I. (eds.). Syntax and Semantics III: Speech Acts. New york: Academic Press. 
Hakes, D.T. 1980. The Development of Metalinguistic Abilities in Children. Berlin: Springer-Verlag.

Halliday, M. \& Hasan, R. 1976. Cohesion in English. London: Longman,

Hammarberg, R, 1976. The Metaphysics of Coarticulation. Journal of phonetics, $4: 353-363$.

Healy, A. 1973. Can Chimpanzees Learn a Phonemic Language? Journal of Psycholinguistic Research, 2:167-9.

Hetzron, R. 1973. Surfacing. Studi italiani di linguistica teorica ed applicata, 2:3-71.

Hill, A. 1958. Introduction to Iinguistic Structures; From Sound to Sentence in English. New York: Harcourt, Brace \& World.

Hockett, Ch. 1958. A Course in Modern Linguistics. New York: Macmillan.

Hockett, Ch. 1968. The State of the Art. The Hague: Mouton.

Hovdhaugen, E. 1980. A History of Western Linguistics. From the beginning to the end of the first millennium A.D. Typescript. Oslo: Dept. of Linguistics.

Hudson, R. 1980a. Constituency and dependency. Linguistics, 18 : $179-i 98$.

Hudson, R. $1980 \mathrm{~b}$. A second attack on constituency: a reply to Dahl. Linguistics, 18:489-504.

Hyltenstam, K. 1978. Variation in Interlanguage Syntax. Working Papers 18 . Department of Linguistics, Lund. 
Jackson, H. 1878. On Affectations of Speech from Disease of the Brain. Reprinted in Selected Writings of Hughlings Jackson (1958), Vol. 2, pp.155-170. New York: Basic Books.

Jakobson, R. 1968. Child Language, Aphasia and Phonological Universals. Transl. by A.R. Keiler of the author's Kindersprache, Aphasie und allgemeine Lautgesetze (1941). The Hague: Mouton.

Jones, E.E. \& Nisbett, R.E. 1971. The Actor and the Observer: Divergent Perceptions of the Causes of Behavior. Morristown, N.J.; General Learning Press.

Johnson-Laird, P.N. 1977. Procedural Semantics. Cognition, $5: 189-214$.

Johnson-Laird, P.N. 1980. Mental Models in Cognitive Science. Cognitive Science, 4:71-115.

Johnson-Laird, P.N. \& Wason. P.C. 1977. (eds.) Thinking. Readings in Cognitive Science. Cambridge: Cambridge University Press.

Katz, Jerrold. 1964. Mentalism in Linguistics. Language, $40: 124-137$.

Kay, P. 1977. Language Evolution and Speech Style. In Blount, B. \& Sanches, M. (eds.) Sociocultural Dimensions of Language Change. New York: Academic Press. 21-33.

Kendon, A. 1980. Gesticulation and Speech: Two Aspects of the Process of Utterance. In Key, M. R. (ed.) The Relationship of Verbal and Nonverbal Communication. The Hague: Mouton. $207-227$.

Kent, R. \& Minifie, F.D. 1977. Coarticulation in Recent Speech Production Models. Journal of Phonetics, 5:115-133. 
Kiparsky, P. 1973. Phonological Representations. In Fujimura, 0. (ec.). Three Dimensions of Linguistic Theory. Tokyo: TEC Company. 1-136.

Kosslyn, S.M. 1980. Image and Mind. Cambridge, Mass.: Harvard University Press.

Kristeva, J. 1969. Semeiotiké. Recherches pour une sémanalyse. Paris: Seuil.

Kuipers, A. 1960. Phoneme and Morpheme in Kabardian. The Hague: Moutor.

Labov, W. 1969. The Logic of Nonstandard English. Georgetown Monographs on Language and Linguistics, Vol. 22:1-31.

Ladefoged, P. 1967. Three Areas of Experimental Phonetics.

Oxford: Oxford University Press.

Lakoff, G. \& Johnson, M. 1980. Metaphors We Live By. Chicago: The University of Chicago Press.

Larsen, P. Harms. 1979. Som sagt, så skrevet? Om forholdet mellem talesprogsnormer og skriftsprogsnormer i offentligheds - og socialisationsteorettsk belysning. In Sprognormer i Norden. Special issue of Nydanske Studier og AImen Kommunikationsteori, Vol. 12. København: Akademisk Forlag.

Levy, D. 1979. Communicative Goals and Strategies: Between Discourse and syntax. In Givón, T. (ed.) Syntax and Semantics, Vo1. 12. New York: Academic Press. 183-210.

Iine11, P. 1979a. Psychological Reality in Phonology. A Theoretical Study. (Cambridge Studies in Iinguistics, 25). Cambridge: Cambridge University Press.

Linel1,P. 1979b. On the Similarity Between Skinner and Chomsky. In Perry (1979):190-199. 
Linel1, P. 1982a. The Concept of Phonological Form and the Activities of Speech Production and Speech Perception. Journal of Phonetics, 10, 37-72.

Linel1, P. 1982b. Speech Errors and the Grammatical Planning of Utterances. Evidence from Swedish. In Koch, W.,Platzack, C. \& Tottie, G. (eds.) Textstrategier $i$ tal och skrift. Stockholm: Almqvist \& Wiksell International. 134-151.

Linel1, P. Reflections on Utterance Production. (In prep.) Linköping: Department of Communication Studies.

Loman, B. \& Järgensen, N. 1971. Manual för analys och beskrivning av makrosyntagmer. Lund: Studentlitteratur.

Lyons, J. 1968. Introduction to Theoretical Linguistics. Cambridge: Cambridge University Press.

Lyons, J. 1977. Semantics. (Two columes). Cambridge: Cambridge University Press.

Lyons, J. 1981. Language and Linguistics. Cambridge: Cambridge University Press.

MacNellage, P. 1979. Speech Production. In Ninth International Congress of phonetic Sciences 1979, Prooceedings.Vol. 1. Copenhagen: Institute of Phonetics, 11-39.

Maher, B. 1972. The language of schizophrenia: a review and interpretation. British Journal of Psychiatry. 120:3-17.

Maher, P, 1969. The Paradox of Creation and Tradition in Gramar: Sound Pattern of a Palimpsest. Language Sciences, 7:15-24.

Malinowski, B. 1949. The Problem of Meaning in Primitive Languages. Supplement I in Ogden,C.K. \& Richards, I.A. (eds.) The Meaning of Meaning. (10. ed.) London: Routledge \& Kegan Paul. 296-336. 
Marslen-Wilson, W. 1976. Linguistic Descriptions and Psychological Assumptions in the study of Sentence Perception. In Wales, R.J. \& Walker, E. (eảs.) New Approaches to Language Mechanisms, Amsterdam: North-Holland. 203-229.

McCawley, J.D. 1980. Review of Newmeyer, F. (1980) Linguistic theory in America: the first quarter-century of transformational generative gramar. Iinguistics, 18:911-930.

McLuhan, M. 1965. Understanding Media: The Extensions of Man. New York: McGraw-Hill.

Merleau-Ponty, M. 1962. Phenomenology of Perception. Transl. by c. Smith of the author's phénaménologie de la perception. London: Routledge \& Kegan Paul.

Miller, G.A. \& Johnson-Laird, P.N. 1976. Language and Perception. Cambridge: Cambridge University Press.

Montague, R. 1974. Formal Philosophy. Edited by R. Thomason. New Haven: Yale University Press.

Mueller, C. 1971. Notes on the Repression of Communicative Behavior. In Dreitzel, H. (ed.) Recent Sociology, No. 2. New York: Macmilian.

Myerson, R. 1975. A Developmental Study of Chilaren's Knowledge of Complex Derived Words in English. Mimeo, Harvard Građuate School of Education.

Naess, A. 1953. Interpretation and Preciseness. A Contribution to the Theory of Communication. Oslo: Det Norske Videnskapsakademi 1 Oslo (Skrifter, II, Hist,-filos. Kl. 1953:1).

Neisser, U. 1976. Cognition and Reality. San Francisco:

W.H. Freeman. 
Nisbett, R.E. \& Decamp Wilson, T. 1977. Telling more than we know: verbal reports on mental reports. Psychological Review, $84: 231-259$.

Oller, K. Wieman, I., Doyle, W. \& Ross, C. 1976. Infant babbling and speech. Journal of Child Language, 3:1-11.

Olsen, S-E. 1979. On the Information-Processing Paradigm in Cognitive Psychology and Psycholinguistics. In Pettersson, Th. (ed.) Papers from the Fifth Scandinavian Conference of Linguistics. Part II. Stockholm: Almqvist \& Wiksell International. 93-108.

01son, D. 1977. From Utterance to Text: The Bias of Language in Speech and Writing. Harvard Educational Review, 47:257-281.

Ong, W. 1959. Latin Language Study as a Renaissance Puberty Rite. Studies in Philology, LVI: 103-124.

Ong, W. 1974. The History and the Future of Verbal Media. In Silverstein, A. (ed.) Human Communication: Theoretical Explorations. Hillsdale, N.J.: Erlbaum. 165-183.

Ortony, A. 1979 (ed.) Metaphor and Thought. Cambridge: Cambridge University Press.

Osgood, Ch. 1979. What is a Language? In Aaronson, D. \& Rieber, R. (eds.). Psycholinguistic Research: Implications and Applications. Hillsdale, N.J.: Erlbaum. 189-228.

Partee, B. Hall. 1971. On the Requirement that Transformations Preserve Meaning. In Flllmore, Ch. \& Langendoen, T. (eds.), Studies in Linguistic Semantics. New York: Holt, Rinehart \& winston. 1-21.

Perrault, R. \& Allen, J. 1980. A Plan-Based Analysis of Indirect Speech Acts. American Journal of Computational Linguistics, 6 , 167-182. 
Perry, Th. 1979. (ed.) Evidence and Argumentation in Linguistics. Berlin: de Gruyter.

Pike, K. 1945. The Intonation of American English. Ann Arbor: The university of Michigan Press.

Pike, K. 1947. Phonemics: A Technique for Reducing Languages to Writing. Ann Arbor: The University of Michigan Press.

Postal, P. 1968. Aspects of Phonological Theory. New York: Harper \& Row.

Pylyshyn, 2. 1973. What the Mind's Eye Tells the Mind's Brain: A Critique of Mental Imagery. Psychologica- Bulletin, 80 . 1-24.

Read, Ch. 1971. Pre-school Children's Knowledge of English Phonology. Harvard Educational Review, 41:1-34.

Reddy, M. 1979. The Conduit Metaphor - A Case of Frame Conflict in our Language about Language. In ortony (1979):284-324.

Reichman, R. 1978. Conversational Coherency. Cognitive Science, $2: 283-327$.

Roedinger, H.III.1980.Memory Metaphors in Cognitive Psychology. Memory \& Cognition, 8: 231-246.

Rosenberg, Sh. 1977 (ed.). Sentence Production; Developments in Research and Theory. Hillsdale, N.J.: L. Erlbaum.

Rommetveit, R. 1978. Language Games, Syntactic Structures and Hermeneutics. In Rommetveit \& Blakar $(1978): 35-76$.

Rommetveit, R. \& Blakar, R. 1978 (eds.). Studies of Language, Thought and Verbal Communication. London: Academic Press.

Ross, J.R. 1967. Constraints on Variables in Syntax. M.I.T. Diss. Reproduced by Indiana University Linguistics Club. 
Rumelhart, D. 1979. Some Problems with the Notion of Literal Meanings. In Ortony $(1979): 78-90$.

Sadock, J. 1979. Figurative Speech and Linguistics. In Ortony $(1979): 46-63$.

Saffran, E.M.,Schwartz, M.F. \& Martin, O.S.M. 1980. Evidence from Aphasia: Isolating the Components of a Production Model. In Butterworth (1980): 221-241.

Sajavaara, K. 1980. Psycholinguistic Models, Second Lanquage Acquisition, and Contrastive Analysis. Reprint. University of JYväskylä: Department of English.

Sajavaara, K. \& Lehtonen, S. 1978. Spoken Language and the Concept of Fluency. In Lautamatti, I. \& Lindqvist, P. (eds.) Focus on Spoken Language (Kielikeskusuutisia, 1). Jyväskylä: Language Centre. 23-57.

Sandig, B. 1973. Zur historischen Kontinuität normativ diskriminierter syntaktischer Muster in spontaner Sprechsprache. Deutsche Sprache, $1: 37-57$.

Saussure, F. de. 1964. (1916). Cours de linguistique générale, Paris: Payot.

Schane, S. 1968. French Phonology and Morphology.Cambridge, Mass.: The M.I.T. Press.

Scribner, S. 1977. Modes of Thinking and Ways of Speaking: Culture and Logic Reconsidered. In. Johnson-Laird \& Wason (1977): 483-500.

Scribner, S. \& Cole, M. 1981. Unpackaging Literacy. In Whiteman (1981): 71-87.

Searle, J. 1969. Speech Acts. Cambridge: Cambridge University Press. 
Searle, J. 1979. Metaphor. In ortony (1979): 92-123.

Steiner, G. 1975. After BabeI. Aspects of Language and Translation. Oxford: Oxford University Press.

Stenlund, S. 1980. Det osägbara. Stockholm: Norstedt.

Stokoe, w.C. 1960. Sign language structure. An outline of the visual communication systems of the American deaf. In Studies in linguistics, Occasional Papers, No. 8. Buffalo, N.Y.

Stokoe, W.C. 1972. Semiotics and Human Sign Languages. The Hague: Mouton.

Storms, M. 1973. Videotape and the Attribution Process: Reversing Actors' and Observers' Points of View. Journal of Personality and Social Psychology, 27: 165-175.

Strömqvist, S. 1980. Speech as Action in the Play of Swedish Three-Year-0lds. Paper No. 3. Stockholm: Child Language Research Institute.

Tannen, D. 1982a. (ed.). Spoken and Written Language: Exploring Orality and Literacy. Norwood, N.J.: Ablex.

Tannen, D. 1982b. (ed.). Coherence in Spoken and Written Discourse. Norwood, N.J.: Ablex.

Teleman, 0. 1980. On language norms in the German Democratio Republic. Review of Hartung, w. (ed.). Normen in der sprachlichen Kommunikation (Berlin, 1977). Linguistics, 18:335-45.

Thavenius, J. 1981. Modersmäl och fadersarv. Svenskämnets traditioner 1 historien och nuet. Järfälla: Symposion Bokförlag. 
Thorndyke, P.W. 1975. Cognitive Structures in Human Story Comprehension and Memory. Unpubl doctoral dissertation. Stanford University, California.

Ullmann, S. 1951. The Principles of Semantics, Oxford: Basic Blackwell.

Vachek, J. 1939. Zum Problem der geschriebenen Sprache. In Traveaux du Cercle Linguistique de Prague, VIII:94 ff.

Vachek, J. 1949. Some Remarks on Writing and Phonetic Transcription. Acta Linguistica, $V: 2,86-93$.

Vennemann, Th. 1968. Die Affrikaten in der generativen Phonologie des Deutschen. Phonetica, 18:65-76.

Volosinov, V.N. 1973. Marxism and the Philosophy of Language. Trans1. by L. Matejka and I.R. Titunik. New York: Seminar Press.

Washabaugh, W. 1980. The role of speech in the construction of reality. Semiotica, 31:197-214.

Wason, P.C. \& Johnson-Laird, P.N. 1972. Psychology of Reasoning: Structure and Content. London: Batsford.

Welin, C.w. 1979. Studies in Computazional Text Comprehension. Monographs from the Institute of Linguistics, 5 , University of stockholm.

Werner, H. \& Kaplan, B. 1963. Symbol Eormation. An OrganismicDevelopmental Approach to Language and the Expression of Thought. New York: John Wiley.

Whiteman, M.F. 1981. (ed.). Writing: The Nature, Development, and Teaching of Written Communication. Vol. 1: Variation in Writing: Functional and Iinguistic-Cultural Differences. Hillsdale, N.J.: Erlbaum. 
Winograd, T. 1975. Frame Representations and the DeclarativeProcedural Controversy. In Bobrow, D.G. \& Collins, A. (eds.) Representation and Understanding: Studies in Cognitive Science. New York:Academic Press.

Winograd, T. 1980. What does it mean to understand language? Cognitive Science, $4: 209-241$.

Woo, N. 1969. Prosody and Phonology. M. T.T. diss. Reproduced by Indiana University Linguistics club.

von Wright, G.H. 1965. And next. Acta Phtlosophia Fennica, Fasc. $16: 293-304$. 



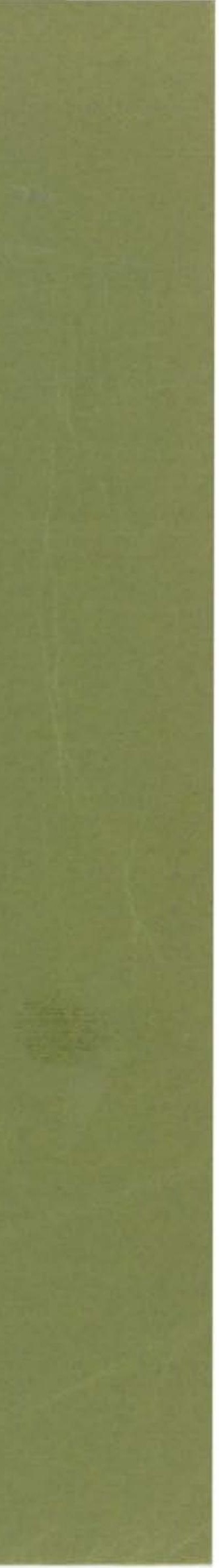


In the Faculty of Arts and Science at the University of Linkoping, Sweden, re search and postgraduate researeh training are conducted within broad problem areas - themes - instead of traditional academic disciplines. There are four themes: Health and Society, Communication Studies, Technology and Social Change, and Water in Environment and Society. Within each theme research is pursued through cooperation between scholars with different scientific backgrounds. Research activity commenced in 1980 and after a period of construction of $6-7$ years each theme will have an establishment of $20-25$ research posts professors, readers etc. $\rightarrow$ and ca. 40 research sudents.

Previous issues:

SIC I Linell, Per. Modeller och metaforer for kommiunikafion. 1982

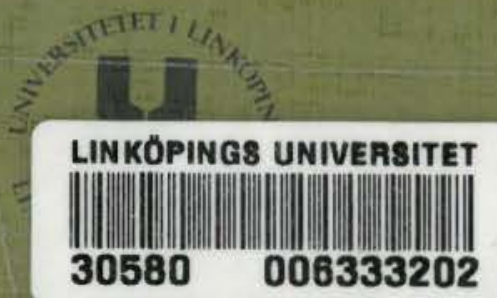

ISSiN $0280-5634$ 